\title{
Unit representation of semiorders II: The general case
}

\author{
Denis Bouyssou ${ }^{1} \quad$ Marc Pirlot $^{23}$
}

July 14, 2020

${ }^{1}$ LAMSADE, UMR 7243, CNRS, Université Paris-Dauphine, PSL Research University, 75016 Paris, France, e-mail: denis.bouyssou@lamsade.dauphine.fr

${ }^{2}$ Université de Mons, rue de Houdain 9, 7000 Mons, Belgium, e-mail: marc.pirlot@umons.ac.be

${ }^{3}$ Corresponding author 


\begin{abstract}
Necessary and sufficient conditions under which semiorders on uncountable sets can be represented by a real-valued function and a constant threshold are known. We show that the proof strategy that we used for constructing representations in the case of denumerable semiorders can be adapted to the uncountable case. We use it to give an alternative proof of the existence of strict unit representations. A direct adaptation of the same strategy allows us to prove a characterization of the semiorders that admit a nonstrict representation.
\end{abstract}

Keywords: semiorder, numerical representation, constant threshold, uncountable sets 


\section{Contents}

1 Introduction 1

2 Notation and previous results 3

2.1 Notation and definitions . . . . . . . . . . . . . . . 3

2.2 Candeal and Induráin's (2010) result . . . . . . . . . . . . . . . 5

$\begin{array}{lll}3 & \text { Another formulation of } s \text {-separability } & \mathbf{6}\end{array}$

3.1 Noses and hollows . . . . . . . . . . . . . . . . . . 6

3.2 A reformulation of $s$-separability . . . . . . . . . . . 8

4 Semiorders admitting a strict unit representation 9

4.1 Results on the partition into maximal indifference classes . . . . . . 10

4.2 Notation . . . . . . . . . . . . . . . . . . . . 11

4.3 Construction of $I_{0,1} \ldots \ldots \ldots \ldots \ldots$

4.4 Construction of $I_{0, k} \ldots \ldots \ldots \ldots \ldots$

4.5 Construction of $I_{-1, k} \ldots \ldots \ldots \ldots \ldots \ldots \ldots$

4.6 Construction of $I_{-l, k} \quad \ldots \ldots \ldots \ldots \ldots$

4.7 Construction of a representation on an $I$-connected component . . . 23

4.8 Assembling representations on $I$-connected components . . . . . . . 25

4.9 Hollows-faithful representations . . . . . . . . . . . . . . . . 26

4.10 Summary of results . . . . . . . . . . . . . . . . . . . . 33

5 Nonstrict unit representations $\quad 33$

5.1 Separability for nonstrict unit representations . . . . . . . . . . . 34

5.2 Characterization of the semiorders admitting a nonstrict unit representation . . . . . . . . . . . . . . . 37

5.3 Both strict and nonstrict unit representation . . . . . . . . . . . 44

$\begin{array}{llr}6 & \text { Discussion } & 44\end{array}$

6.1 Summary ............................. 44

6.2 Some directions for further work . . . . . . . . . . . . . . 47 


\section{Introduction}

Semiorders were introduced as a way of representing stimuli intensities as they are perceived by a subject. A stimulus is not perceived as more intense than another, unless it is more intense by at least some minimal value called just noticeable difference (JND). The relation on a set of stimuli, comparing their intensities, has the peculiarity that indifference (i.e., stimuli indistinctness) may be intransitive. Luce (1956) defined and studied relations allowing to capture such comparisons, that he called semiorders.

All such relations, provided they are defined on finite sets of objects (e.g., stimuli), admit a numerical representation using real numbers and a positive constant threshold. This constant threshold is closely related to the just noticeable difference. More recently, authors have considered semiorders on denumerable (i.e., finite or countably infinite) and on uncountable sets of objects, respectively. Not all semiorders on infinite sets can be represented by a value function and a constant threshold. Manders (1981) and Beja and Gilboa (1992) have given an additional necessary and sufficient condition guaranteeing that semiorders on denumerable sets can be represented in this way. Bouyssou and Pirlot (2020a) give an alternative proof of this result. The latter proof is, in a sense, constructive. It relies on a double decomposition of the set of objects. This set is first decomposed into connected components of the indifference relation. Second, each connected component is partitioned into maximal indifference classes. One of these classes is selected as a "baseline" in which a representative (which we call "ghost") of each other object is inserted in an appropriate order. Finally, using a numerical representation of the order on the baseline, we construct a numerical representation of the semiorder with unit threshold. In the present paper, we follow the same ideas of proof to deal with uncountable sets of objects ${ }^{1}$.

There are actually (at least) two inequivalent ways of defining constant threshold real valued numerical representations of semiorders. Scott and Suppes (1958) consider numerical representations $(u, k)$ consisting of a real-valued function $u$ defined on the set of objects and a constant threshold $k>0$. The fact that stimulus $x$ is definitely perceived as more intense than stimulus $y$ is represented by the following strict inequality: $u(x)>u(y)+k$. The threshold $k$ can thus be interpreted as the largest unnoticeable difference. Alternatively, it can be decided to represent the same fact by the nonstrict inequality: $u(x) \geq u(y)+k$. Here $k$ can be interpreted as the least noticeable difference. We shall call the former type of representation strict; the latter shall be called nonstrict. While the existence of one type of representation implies that of the other type for semiorders on finite

\footnotetext{
${ }^{1}$ We emphasize that the present paper is self-contained and can be read independently of the one dealing with denumerable semiorders.
} 
sets (Pirlot and Vincke, 1997, p. 72) and countably infinite sets (Beja and Gilboa, 1992, Th. 3.8, p. 436), this is no longer the case for semiorders on uncountable sets.

In the general case, two different sets of necessary and sufficient conditions for the existence of a strict representation (also called a Scott-Suppes representation) have been established by Beja and Gilboa (1992) and by Candeal and Induráin (2010) (see also Giarlotta and Watson, 2016, who derive Candeal and Induráin's result from of a more general analysis). Both characterizations have in common a condition that guarantees the existence of a strict representation in the denumerable case (we call it the Bounded P-chain condition below). On the top of it, Candeal and Induráin impose a new separability condition that they name $s$-separability. In this work, we factorize the $s$-separability condition into the usual Debreu-separability of the trace (i.e., of the complete preorder induced by the semiorder on the set of objects) and another condition that is easily interpretable. This analysis clarifies the relationships between Candeal and Induráin's and Beja and Gilboa's characterizations. Then, using the same strategy of proof as in Bouyssou and Pirlot (2020a), we give an alternative proof of Candeal and Induráin (2010)'s result. We shall discuss later the interest of this method.

Semiorders on uncountable sets that admit a nonstrict representation have been characterized by Beja and Gilboa (1992) (while Candeal and Induráin (2010) did not tackle this question). We establish another characterization, relying on the same proof strategy as before, using a condition similar to $s$-separability. The new type of separability can also be factorized into Debreu-separability of the trace and another easily interpretable condition. The latter also clarifies the relationships with Beja and Gilboa's characterization. Note that the conditions that have to be added to Debreu-separability for obtaining either strict or nonstrict representations use the notions of noses and hollows that were fruitful in the study of finite semiorders (Balof et al., 2013, Doignon, 1988, Pirlot, 1990, 1991).

The paper is organized as follows. In the next section, we introduce the notation and recall the characterization by Candeal and Induráin (2010). In Section 3, we factorize one of the conditions used by Candeal and Induráin (2010) in their characterization theorem into simpler ones. In Section 4, we give an alternative proof of the existence of a strict unit representation for semiorders under three simple conditions. These are necessary and sufficient. We do the same in Section 5 for semiorders admitting nonstrict unit representations. We conclude with a discussion. 


\section{Notation and previous results}

\subsection{Notation and definitions}

We use the same notation as in Bouyssou and Pirlot (2020a). Let $S$ be a binary relation on a set $X$. The relation $S$ is a semiorder if it is complete ${ }^{2}(x S y$ or $y S x$, for all $x, y \in X)$, Ferrers $(x S y$ and $z S w \Rightarrow x S w$ or $z S y$, for all $x, y, z, w \in X$ ) and semi-transitive $(x S y$ and $y S z \Rightarrow x S w$ or $w S z$, for all $x, y, z, w \in X)$. In the sequel, we shall often write the semiorder $S$ as a pair $(P, I)$ of relations, where $P$ (resp. $I$ ) denotes the asymmetric (resp. symmetric) part of $S . P$ is a partial order on $X$, i.e., an asymmetric and transitive relation, which is also Ferrers and semitransitive. $I$ is called the indifference relation; it is reflexive and symmetric but not necessarily transitive. A complete preorder on $X$ is a complete, reflexive, and transitive relation. It is a particular case of a semiorder. A linear order (or total order) on $X$ is a complete, antisymmetric and transitive relation.

The trace $\succsim_{S}$ of a semiorder $S$ on $X$ is the relation defined as follows: for all $x, y \in X, x \succsim_{S} y$ if for all $z \in X, y S z$ implies $x S z$ and $z S x$ implies $z S y$. The subscript $S$ will be omitted whenever there is no ambiguity regarding the associated semiorder. We use $\succ, \precsim, \prec, \sim$ as is usual. It is well-known that the trace of a semiorder is a complete preorder. Two elements $x, y \in X$ such that $x \succsim y$ and $y \succsim x$, i.e., $x \sim y$, are said to be equivalent. If $x \sim y$, then, for all $z \in X$, we have $z S x$ iff $z S y$ and $x S z$ iff $y S z$.

Definition 1 (Strict and nonstrict unit representations)

A strict unit representation of the semiorder $S=(P, I)$ on the set $X$ is a function $u$ from $X$ to $\mathbb{R}$ such that, for all $x, y \in X$,

$$
\begin{array}{cl}
u(x)>u(y)+1 & \text { if } \quad x P y \\
-1 \leq u(x)-u(y) \leq 1 & \text { if } \quad x I y
\end{array}
$$

A nonstrict unit representation of the semiorder $S=(P, I)$ on the set $X$ is a function $u$ from $X$ to $\mathbb{R}$ such that, for all $x, y \in X$,

$$
\begin{array}{cl}
u(x) \geq u(y)+1 & \text { if } \quad x P y \\
-1<u(x)-u(y)<1 & \text { if } \quad x I y
\end{array}
$$

Strict unit representations are the special case of the Scott-Suppes representations (Candeal and Induráin, 2010), in which $k=1$. When dealing with the existence of a Scott-Suppes representation, it is not restrictive to focus on strict unit representations because the latter exists iff the former exists. Nonstrict unit representations

\footnotetext{
${ }^{2}$ Imposing that $S$ is reflexive, instead of complete, would suffice since relations that are both reflexive and Ferrers are complete.
} 
have been less studied (Beja and Gilboa, 1992, is an exception), probably because, a semiorder defined on a denumerable set admits a strict representation iff it admits a nonstrict representation. This is no longer the case in the uncountable case as will be shown in Section 5. In contrast, for other ordered structures, the conditions for the existence of nonstrict representations are well-known. It is the case for biorders (Aleskerov et al., 2007, Beja and Gilboa, 1992, Doignon et al., 1984) and for interval orders (see Aleskerov et al., 2007, Ch. 6).

We shall only consider strict and nonstrict representations that assign the same value to equivalent elements of $X$ w.r.t. the trace $\succsim$ (i.e., regular representations, Roberts and Franke, 1976). Consequently, we may assume henceforth that the equivalence class of each element of $X$ w.r.t. the trace of the semiorder is reduced to a singleton. In other words, for all $x, y \in X, x \succsim y$ and $y \succsim x$ imply $x=y$. This assumption can be made w.l.o.g. (Candeal and Induráin, 2010, Lemma 3.2). Therefore, the trace $\succsim$ is a linear order on $X$. Its asymmetric part is denoted by $\succ$ and its symmetric part by $\sim$.

In Bouyssou and Pirlot (2020a, Section 2.2) (see also Manders, 1981), we have shown that every semiorder $S=(P, I)$, be it on a denumerable or uncountable set, can be decomposed into connected components of the indifference relation $I$. An $I$-connected component of $(X, S)$ is a maximal subset $Y$ of $X$ such that, for each pair $x, y \in Y$, there is an $I$-chain joining them, i.e., there are $x_{1}, x_{2}, \ldots, x_{n} \in$ $Y$ such that $x I x_{1} I x_{2} I \ldots I x_{n} I y$. Furthermore, the relation $P$ induces a linear order (called "macro-ordering" by Giarlotta and Watson (2016)) on the set of $I$-connected components of $(X, S)$.

A necessary condition for the existence of a strict or a nonstrict unit representation of a semiorder is the Bounded P-chain condition (see Property 1 below). Before stating it, we need some definitions.

A $P$-chain is a family of elements $\left(x_{j}, j \in J\right)$ indexed by a set $J \subseteq \mathbb{Z}$ of consecutive integers, such that $x_{j} P x_{j+1}$, for all $j \in J$ with $j+1 \in J$. The $P$-chain is bounded if there are elements $a$ and $b$ such that $b P x_{j} P a$, for all $j \in J$.

\section{Property 1 (Bounded $\boldsymbol{P}$-chain condition)}

Every bounded P-chain is finite.

The semiorder $S$ on the set $X$ satisfies the Bounded $P$-chain condition if every $P$ chain $\left(x_{j}, j \in J\right)$ ( $J$ a set of consecutive integers), that is contained in an interval $[a, b]$ of $(X, \succsim)$ has only finitely many elements $(|J|<\infty)$ (Bouyssou and Pirlot, 2020a, Cor. 53).

Bouyssou and Pirlot (2020a, Proposition 54) establishes that the Bounded Pchain condition is equivalent to the necessary and sufficient condition of Manders (1981) for the existence of a unit representation of a semiorder on a denumerable set. The Bounded P-chain condition is also equivalent to Beja and Gilboa 
(1992)'s condition (Bouyssou and Pirlot, 2020a, Sections 5.2 and 5.3). Candeal and Induráin (2010) call this property regularity ${ }^{3}$. If $X$ is $I$-connected, it is clear that $(X, S)$ always satisfies the Bounded $P$-chain condition (Bouyssou and Pirlot, 2020a, Proposition 54).

The strategy followed in Bouyssou and Pirlot (2020a) to show the existence of a unit representation for a semiorder defined on a denumerable set is to show the existence of such a representation on each $I$-connected component of the semiorder and then, assuming the Bounded P-chain condition, to show that it is possible to assemble these representations into a representation of the whole semiorder. We shall adopt the same strategy in case the semiorder is defined on an uncountable set. Under an additional condition, we show that a strict unit representation exists on each $I$-connected component and we assemble these representations assuming the Bounded $P$-chain condition ${ }^{4}$. This can be done also for nonstrict unit representations by substituting the additional condition for strict representations by an additional condition that is suitable for nonstrict representations.

In the next subsection, we recall Candeal and Induráin's (2010) result and the additional condition that they have to impose on semiorders in order to prove the existence of a strict unit representation.

\subsection{Candeal and Induráin's (2010) result}

Candeal and Induráin (2010) assume the following additional condition that they call $s$-separability.

\section{Definition 2 ( $s$-separability)}

A semiorder $S=(P, I)$ on $X$ is s-separable if there is a denumerable set $E, E \subseteq X$, such that, for all $a, b \in X$ with $a P b$, there are

$$
\begin{aligned}
& c \in E \quad \text { such that } a P c \succsim b \\
& \text { and } d \in E \text { such that } a \succsim d P b
\end{aligned}
$$

The main result in (Candeal and Induráin, 2010, Theorem 3.6) can be rephrased as follows.

Theorem 3 (Candeal and Induráin (2010))

$A$ semiorder $S$ on a set $X$ admits a strict unit representation iff it satisfies the Bounded P-chain condition and is s-separable.

\footnotetext{
${ }^{3}$ This regularity property should not be confused with the regularity property of representations introduced by Roberts and Franke (1976)

${ }^{4}$ As observed in Bouyssou and Pirlot (2020a, Remark 64) assembling representations on $I$ connected components of a semiorder is possible for uncountable semiorders, under the same conditions as for denumerable semiorders.
} 
Notice that the trace of an $s$-separable semiorder is $d$-separable or Debreu-separable (Candeal and Induráin, 2010, Lemma 3.4), which is a condition guaranteeing the existence of a numerical representation of the trace (i.e., the existence of a function $v: X \rightarrow \mathbb{R}$ such that $x \succsim y$ iff $v(x) \geq v(y)$ ). It can be assumed w.l.o.g. that a representation of the semiorder also represents its trace. Indeed, a numerical representation $u$ of the semiorder must give distinct values to elements that are not equivalent w.r.t. the trace. Therefore $u$ must represent the trace since we assumed that no elements are equivalent w.r.t. the trace. We recall the definition of Debreu-separability below.

\section{Definition 4 ( $d$-separability)}

A semiorder $S=(P, I)$ is d-separable if its trace $\succsim i s d$-separable. The trace is $d$-separable if it admits a denumerable order-dense set, i.e., there is a denumerable set $D \subseteq X$, such that, for all $a, b \in X$ with $a \succ b$, there is $d \in D$, such that $a \succsim d \succsim b$.

The $d$-separability of the trace is a necessary condition for the existence of both a strict and a nonstrict (see Section 5) representation of a semiorder.

\section{Another formulation of $s$-separability}

In this section we revisit the $s$-separability condition and factorize it into $d$ separability and another condition. The latter is expressed in terms of noses, a notion introduced in Pirlot $(1990,1991)$, together with that of hollows. These have proved useful in the study of the unit representations of finite semiorders (Balof et al., 2013, Doignon, 1988, Pirlot, 1990, 1991).

The results in this section have appeared in Section 3.2 of Bouyssou and Pirlot (2020b). For the ease of reading, we recall them here.

\subsection{Noses and hollows}

Let $S=(P, I)$ be a semiorder on $X$. A nose of $S$ is a pair $(a, b)$ such that $a P b$ and $a$ is the least element (w.r.t. $\succ$ ) which is preferred to $b$ and $b$ is the greatest element (w.r.t. $\succ$ ) which $a$ is preferred to. A hollow is a pair $(a, b)$, with $a I b$ and $a \succsim b$ such that $a$ is the greatest element (w.r.t. $\succ$ ) which is indifferent to $b$ and $b$ is the least element (w.r.t. $\succ$ ) which is indifferent to $a$. More formally, we have:

\section{Definition 5 (Noses and Hollows)}

The ordered pair $(a, b) \in X \times X$ is a nose of $S=(P, I)$ if $a P b$ and there is no $c \in X$ such that $a P c \succ b$ and there is no $d \in X$ such that $a \succ d P b$.

The ordered pair $(a, b) \in X \times X$ is a hollow of $S=(P, I)$ if $a I b, a \succ b$, and there is no $c \in X$ such that $b I c \succ a$ and there is no $d \in X$ such that $b \succ d I a$. 


\section{Remark 6}

A hollow can be equivalently defined in terms of the relation $S$. The pair $(a, b)$, with $a \succ b$, is a hollow iff $b S a$, for all $d \prec b, \operatorname{Not}[d S a]$, and for all $c \succ a, \operatorname{Not}[b S c]$. Indeed, since $a \succ b$, there is $c$ with $c \succ a$ and $b I c$ iff there is $c$ with $\succ a$ and $b S c$; likewise, there is $d$ with $b \succ d$ and $d I a$ iff there is $d$ with $b \succ d$ and $d S a$.

Noses play a special role w.r.t. $s$-separability as shown by the following lemma.

\section{Lemma 7}

If the semiorder $S=(P, I)$ on $X$ is s-separable by the denumerable set $E$, then a and $b$ belong to $E$ whenever $(a, b)$ is a nose.

\section{PROOF}

Let $(a, b)$ be a nose, so that $a P b$. By the $s$-separability property, there is $c \in E$ such that $a P c \succsim b$. By definition of a nose, we have $c=b$ and therefore, $b \in E$. Using $s$-separability, there is also $d \in E$ such that $a \succsim d P b$, which implies $a=d$ and $a \in E$ since $(a, b)$ is a nose.

Before presenting conditions equivalent to $s$-separability, we need to establish some auxiliary results related to variants of noses.

\section{Definition 8 (Half-noses)}

The pair $(a, b) \in X \times X$ is a lower half-nose (l-h-nose) of $S=(P, I)$ if aPb and there is no $c \in X$ such that $a P c \succ b$. The pair $(a, b)$ can be a nose. If it is not, we say it is a proper l-h-nose if it is a l-h-nose and there is $d \in X$ such that $a \succ d P b$. The pair $(a, b) \in X \times X$ is an upper half-nose (u-h-nose) of $S=(P, I)$ if aPb and there is no $d \in X$ such that $a \succ d P b$. The pair $(a, b)$ can be a nose. If it is not, we say it is a proper $u$-h-nose if it is an $u$-h-nose and there is $c \in X$ such that $a P c \succ b$. We denote by LHN (resp. UHN) the set of right endpoints b (resp. left endpoints a) of all proper l-h-noses (resp. u-h-noses) $(a, b)$.

The notion of lower half-nose is closely related to that of $P$-gap-edge point (Beja and Gilboa, 1992, Definition P6 (a), p. 438). The element $b \in X$ is a $P$-gap-edgepoint if and only if there is $a \in X$ such that $(a, b)$ is a lower half-nose. We have the following result.

\section{Lemma 9}

If the semiorder $S=(P, I)$ is d-separable, then the sets $L H N$ and $U H N$ are denumerable.

\section{PROOF}

We give the proof for $L H N$. The case of $U H N$ is similar.

Let $(a, b)$ be a proper l-h-nose. We define the set $N(b)=\{x \in X: x P b$ and $\forall c \succ$ $b, N o t[x P c]\}$. In other words, for all $x \in N(b),(x, b)$ is a l-h-nose. It is clear 
that $a \in N(b)$. Moreover, since $(a, b)$ is proper, $N(b)$ contains at least one other element $d$ such that $a \succ d P b$. We now prove that $N(b)$ is an interval w.r.t. $\succ$. Let $x, x^{\prime} \in N(b)$. If $x^{\prime \prime}$ is such that $x \succ x^{\prime \prime} \succ x^{\prime}$, then $x^{\prime \prime} \in N(b)$. Indeed, $x^{\prime \prime} P b$ since $x^{\prime} P b$ and for all $c \succ b, \operatorname{Not}\left[x^{\prime \prime} P c\right]$ since Not $[x P c]$. Let $(a, b)$ and $\left(a^{\prime}, b^{\prime}\right)$ be two proper l-h-noses, with $b \neq b^{\prime} \in L H N$. It is clear that the associated intervals $N(b)$ and $N\left(b^{\prime}\right)$ are disjoint. Each of these intervals contains at least two distinct points and therefore at least an element from the denumerable set $D$ that $d$-separates $S=(P, I)$. Consequently, the set $L H N$ is denumerable.

\subsection{A reformulation of $s$-separability}

We are in position to prove an equivalent formulation for $s$-separability.

\section{Proposition 10}

A semiorder $S=(P, I)$ on $X$ is s-separable iff $\succsim$ is $d$-separable and the set of noses is denumerable.

\section{PROOF}

Assume that the semiorder is $s$-separable. By Lemma 7 , the set of noses is denumerable. The $s$-separability property implies that $\succsim$ is $d$-separable (Candeal and Induráin, 2010, Lemma 3.4). We include the proof of this for completeness. Let $x, y \in X$ be such that $x \succ y$. There is $z \in X$ such that $x P z$ and $z S y$ and/or $w \in X$ such that $w P y$ and $x S w$. In the former case, $s$-separability entails that there is $d \in E$ such that $x \succsim d P z$ and, since $z S y$, we have $x \succsim d \succ y$. In the latter case, there is $c \in E$ such that $w P c \succsim y$ and, since $x S w$, we have $x \succ c \succsim y$.

Reciprocally, let $D$ be a denumerable set that $d$-separates $\succ$. Let $x, y \in X$ be such that $x P y$. If $(x, y)$ is not a nose,

1. either there is $x^{\prime} \prec x$ such that $x^{\prime} P y$

2. or there is $y^{\prime} \succ y$ such that $x P y^{\prime}$.

In Case 1 , by the $d$-separability of $\succ$, there is $d \in D$ such that $x^{\prime} \precsim d \precsim x$. Therefore we have $x \succsim d P y$. Further, there are two cases. Either there is $y^{\prime} \succ y$ such that $x P y^{\prime}$ or for all $y^{\prime} \succ y$, we have Not $\left[x P y^{\prime}\right]$. In the former case, $d$ separability implies that there is $c \in D$ such that $y^{\prime} \succsim c \succsim y$. Then, we have $x P c \succsim y$. Otherwise, $(x, y)$ is a proper l-h-nose. In order to have $c \in E$ such that $x P c \succsim y$, we set $c=y$ and include the denumerable set $L H N$ of right endpoints of the proper l-h-noses in $E$.

In Case 2, by the $d$-separability of $\succ$, there is $c \in D$ such that $y^{\prime} \succsim c \succsim y$. Therefore we have $x P c \succsim y$. Further, either there is $x^{\prime} \prec x$ such that $x^{\prime} P y$ or for all $x^{\prime} \prec x$, we have $\operatorname{Not}\left[x^{\prime} P y\right]$. In the former case, $d$-separability implies that there is $d \in D$ such that $x^{\prime} \precsim d \precsim x$. Otherwise, $(x, y)$ is a proper $\mathrm{u}$-h-nose. In order 
to have $d \in E$ such that $x \succsim d P y$, we set $d=x$ and include the denumerable set $U H N$ of left endpoints of the proper u-h-noses in $E$.

Finally, by considering $E$ as the union of $D, L H N, U H N$ and the set of elements $a, b$ such that $(a, b)$ is a nose, which is denumerable by hypothesis, we obtain a denumerable set $E$, which $s$-separates the semiorder $(P, I)$.

\section{Remark 11}

It is easy to show that having a denumerable set of noses is a necessary condition for a semiorder to have a strict unit representation. Indeed, assume that $u$ is a strict unit representation of the semiorder $S=(P, I)$ and $(a, b)$ is a nose of $S$. Since $a P b$, we have $u(a)>u(b)+1$. Let $\varepsilon_{a b}$ be the positive number $u(a)-u(b)-1$. By definition of a nose, there is no element $c \neq b$ such that $a P c \succ b$ and therefore, there is no $c$ such that $u(c) \in] u(b), u(a)-1]$, an interval of length $\varepsilon_{a b}>0$. To each nose $(a, b)$ is associated such an interval of positive length and all these intervals are disjoint. Since there is only a denumerable number of disjoint intervals of positive length in $\mathbb{R}$, the number of noses is denumerable.

\section{Remark 12}

For proving the existence of a strict unit representation, we shall use $d$-separability and the condition that the number of noses is denumerable, instead of $s$-separability. In the proof, we shall only use the denumerable set $D$ that is dense in the trace $\succsim$ and the denumerable set of noses endpoints. We do not need to add the half-noses or the half-hollows as we had to do in the second part of the proof of Proposition 10. In other words, we do not use all the points in the set $E$ involved in the $s$-separability property (see Definition 2). In the same vein, we do not need to impose that the set of all $P$-gap-edge-points (Beja and Gilboa, 1992, p. 438) is denumerable. Only the cardinality of the set of $P$-gap-edge-points that correspond to noses needs to be controlled. Our condition that the set of noses has to be denumerable refines both the $s$-separability condition and the denumerability of the set of P-gap-edge-points (Beja and Gilboa, 1992, Theorem 4.5 (a), p. 439). Its interpretation is straightforward and it bridges the gap between the conditions imposed by Candeal and Induráin (2010) and those imposed by Beja and Gilboa (1992). A similar analysis will be helpful in the study of nonstrict representations (see Section 5.1).

\section{Semiorders admitting a strict unit representa- tion}

In this section, we adapt the method proposed in Bouyssou and Pirlot (2020a) for building a unit representation to the case of uncountable semiorders. In the denumerable case, we select an initial maximal indifference class $I_{0}$ of an $I$-connected 
component of the semiorder and we insert a "ghost" of each other element of this connected component at an appropriate position in $I_{0}$. The set of all ghosts is denoted $\tilde{I}_{0}$ and $\succsim_{\varphi}$ is a preorder on $\tilde{I}_{0}$ extending the trace on $I_{0}$. This insertion procedure has to be refined in the uncountable case. The main constraint is to insert the ghosts of all elements while ensuring the $d$-separability of the complete preorder $\succsim_{\varphi}$ on the set of ghosts $\tilde{I}_{0}$. Moreover, in contrast with the denumerable case, it is no longer possible to insert ghosts sequentially since $X$ is uncountable. Notice that, unlike in the denumerable case, we shall not attempt to keep the possibility of generating all possible unit representations. We concentrate on proving there is at least one.

\subsection{Results on the partition into maximal indifference classes}

We assume that the semiorder $(X, S)$ has been decomposed into its $I$-connected components. In Bouyssou and Pirlot (2020a, Section 3), we showed that any Iconnected component of a semiorder can be partitioned into maximal indifference classes. This was proved in the general case, without assuming that the semiorder is defined on a denumerable set. Such a partition gives a particular role to a baseline maximal indifference class $I_{0}$. This partition is not unique in general, but the results that follow are valid for any such partition.

Let $\mathcal{D}$ be any $I$-connected component of the semiorder $S=(P, I)$ on the set $X$. We abuse notation also using $S=(P, I)$ for denoting the restriction of the semiorder to the set $\mathcal{D} \subseteq X$. Let $\left(I_{m}, m \in M\right)$, with $M \subseteq \mathbb{Z}, M \ni 0$, be a partition of $\mathcal{D}$ into maximal indifference classes, as described in Bouyssou and Pirlot (2020a, Section 3). We shall also use the more explicit notation $\ldots, I_{-l}, \ldots, I_{-1}, I_{0}$, $I_{1}, \ldots, I_{k}, \ldots$ for this partition, with indices $k, l \geq 0$ such that $k \in M$ and $-l \in M$. Note that the set of indices $M$ may be bounded or unbounded. We recall below Bouyssou and Pirlot (2020a, Proposition 23) that collects useful properties of such a partition.

\section{Proposition 13}

The sets $\left(I_{m}, m \in M\right)$ have the following properties:

1. They are disjoint nonempty convex subsets of $\mathcal{D}$.

2. Their elements are pairwise indifferent, i.e., for all $x, y \in I_{m}$, we have $x I y$.

3. They form an ordered partition w.r.t. $\succ$, i.e. for all $x \in I_{m-1}$ and $z \in I_{m}$, we have $z \succ x$.

4. For all $m \geq 0$ for which $I_{m}$ and $I_{m+1}$ exist, for all $w \in I_{m+1}$, there is $z \in I_{m}$ such that we have $w P z$. 
5. For all $m<0$ for which $I_{m}$ and $I_{m+1}$ exist, for all $v \in I_{m}$, there is $z \in I_{m+1}$ such that we have zPv.

6. For all $m \in M$ for which $I_{m}$ and $I_{m+2}$ exist, for all $w \in I_{m+2}$, for all $v \in I_{m}$, we have $w P v$.

7. $\mathcal{D}=\cup_{m \in M} I_{m}$.

In the process of constructing a unit representation, we make the hypothesis that the trace $\succsim$ of the semiorder $S$ on $X$ is $d$-separable and that the semiorder has a denumerable set of noses. These properties of the semiorder on $X$ are inherited by its restriction to each $I$-connected component. The trace of the restriction of the semiorder to an $I$-connected component is the restriction of the trace of the semiorder to this component (because all elements in an $I$-connected component compare identically to the elements of the other components).

Let $E=E^{\prime} \cup E^{\prime \prime}$ denote the union of $E^{\prime}$, an order-dense denumerable subset w.r.t. $\succsim$, and the set $E^{\prime \prime}$ of all noses endpoints. The set $E$ is thus at most denumerable.

\subsection{Notation}

In order to describe the construction process of a strict unit representation, we need to introduce some notation and definitions.

Ordered bipartitions play an important role in the sequel. Therefore, we recall some precise definitions (following Bridges and Mehta, 1995, p. 17). Let $\succsim$ be a linear order on a set $Y$ and $\succ$ its asymmetric part. We call $(A, B)$ an ordered bipartition of $Y$ if $A \cap B=\varnothing, A \cup B=Y$ and $x \succ y$ for all $x \in A, y \in B$. One of the classes may be empty. An ordered bipartition $(A, B)$, with $A, B \neq \varnothing$,

- is a jump if $A$ has a least element and $B$ has a greatest element;

- is a cut if either $A$ has least element or $B$ has a greatest element (but not both);

- is a gap if neither $A$ has a least element nor $B$ has a greatest element.

Note that, in the absence of ambiguity, we shall write "bipartition" for "ordered bipartition" 5 .

Each element $x \in I_{m}$ determines a bipartition $\left(A_{x}^{m-1}, B_{x}^{m-1}\right)$ of $I_{m-1}$ (provided $I_{m-1}$ exists) and a bipartition $\left(C_{x}^{m+1}, D_{x}^{m+1}\right)$ of $I_{m+1}$ (provided $I_{m+1}$ exists). We have (see also Figure 1:

\footnotetext{
${ }^{5}$ We shall not adopt the term "decomposition", which is used by Bridges and Mehta (1995) as synonymous to "ordered bipartition" because we use "decomposition" for other purposes.
} 
- $A_{x}^{m-1}=\left\{y \in I_{m-1}: x I y\right\}$;

- $B_{x}^{m-1}=\left\{y \in I_{m-1}: x P y\right\}$;

- $C_{x}^{m+1}=\left\{y \in I_{m+1}: y P x\right\}$;

- $D_{x}^{m+1}=\left\{y \in I_{m+1}: y I x\right\}$

For $m>0, B_{x}^{m-1}$ is non-empty; for $m \leq 0, C_{x}^{m+1}$ is non-empty. This follows from Proposition 13.4 and 13.5.

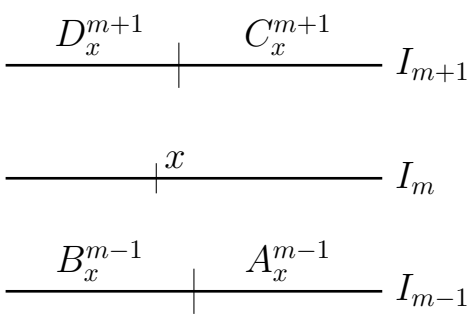

Figure 1: Partitions determined by an element $x \in I_{m}$

The construction of a representation proceeds by selecting a baseline maximal indifference class $I_{0}$ and inserting dummies of the elements of $I_{m}, m \in M \backslash\{0\}$ into $I_{0}$ at an "appropriate location", i.e., by positioning the dummies among the elements of $I_{0}$. In this way, we shall extend the trace $\succsim$ on $I_{0}$ into a complete preorder on $I_{0}$ and the dummies of all other elements in $\mathcal{D}$. We shall also refer to the dummies as ghosts (like in Bouyssou and Pirlot, 2020a). We recall that $E$ denotes a denumerable set that $d$-separates the trace $\succsim$ and contains all noses endpoints. Here is some notation that will be used in the sequel:

$\varphi_{m}\left(I_{m}\right)$, for $m \in M \subseteq \mathbb{Z}$, is the set of ghosts of the elements in $I_{m}$;

$\varphi_{m}(x)$, for $m \in M, x \in I_{m}$, is the ghost of the element $x$ in $I_{m}$;

$\varphi_{0}\left(I_{0}\right)=I_{0} ; \varphi_{0}(x)=x$, for all $x \in I_{0} ;$

$I_{0,1}=I_{0} \cup \varphi_{1}\left(I_{1}\right) ;$

$I_{0, k}=I_{0} \cup \varphi_{1}\left(I_{1}\right) \cup \ldots \cup \varphi_{k}\left(I_{k}\right)$, for $k \in M, k>0$;

$I_{-l, k}=I_{0} \cup \varphi_{1}\left(I_{1}\right) \cup \ldots \cup \varphi_{k}\left(I_{k}\right) \cup \varphi_{-1}\left(I_{-1}\right) \ldots \cup \varphi_{-l}\left(I_{-l}\right)$, for $k, l \in M, k, l>0$;

$E_{m}=E \cap I_{m}$

$E_{0,1}=E_{0} \cup \varphi_{1}\left(E_{1}\right) ;$ 
$E_{0, k}=E_{0} \cup \varphi_{1}\left(E_{1}\right) \cup \ldots \cup \varphi_{k}\left(E_{k}\right)$, for $k \in M, k>0$;

$E_{-l, k}=E_{0} \cup \varphi_{1}\left(E_{1}\right) \cup \ldots \cup \varphi_{k}\left(E_{k}\right) \cup \varphi_{-1}\left(E_{-1}\right) \ldots \cup \varphi_{-l}\left(E_{-l}\right)$, for $k, l \in M, k, l>0$;

$\succsim_{0,1}$ is a complete preorder on $I_{0,1}$, with $\succ_{0,1}$, its asymmetric part, and $\sim_{0,1}$, its symmetric part;

$\succsim_{0, k}$ is a complete preorder on $I_{0, k}$, with $\succ_{0, k}$, its asymmetric part, and $\sim_{0, k}$, its symmetric part, for $k \in M, k>0$;

$\succsim_{-l, k}$ is a complete preorder on $I_{-l, k}$, with $\succ_{-l, k}$, its asymmetric part, and $\sim_{-l, k}$, its symmetric part, for $k, l \in M, k, l>0$.

\subsection{Construction of $I_{0,1}$}

The set $I_{0,1}$ is the union of $I_{0}$ and the set of the ghosts of the elements of $I_{1}$. The ghost $\varphi_{1}(x)$ for $x \in I_{1}$ is distinct from all elements of $I_{0}$. It is defined as being positioned between the two classes of the bipartition $\left(A_{x}^{0}, B_{x}^{0}\right)$, i.e., above all elements in $B_{x}^{0}$ and below all elements in $A_{x}^{0}$. More precisely, we define the relation $\succsim_{0,1}$ on $I_{0,1}$ as an extension of the order $\succsim$ on $I_{0}$ which satisfies $a \succsim_{0,1} \varphi_{1}(x) \succ_{0,1} b$, for all $a \in A_{x}^{0}$ and $b \in B_{x}^{0}$.

In case several elements of $I_{1}$ determine the same bipartition, their ghosts all have to be inserted in between the classes of the bipartition. Let $J(x)$ denote the set of all elements in $I_{1}$ which determine the same bipartition $\left(A_{x}^{0}, B_{x}^{0}\right)$ as $x$. For all $x_{1}, x_{2} \in J(x)$, with $x_{1} \succ x_{2}$, we have $a \succsim_{0,1} \varphi_{1}\left(x_{1}\right) \succ_{0,1} \varphi_{1}\left(x_{2}\right) \succ_{0,1} b$, for all $a \in A_{x}^{0}$ and $b \in B_{x}^{0}$. At this stage, the only case in which we place a ghost $\varphi_{1}\left(x_{1}\right)$ and an element $b$ of $I_{0}$ in the same equivalence class of the relation $\succsim_{0,1}$ is when $\left(x_{1}, a\right)$ is a hollow. In such a case, we set $a \sim_{0,1} \varphi_{1}\left(x_{1}\right)$. Note that we are not forced to make these elements equivalent in all $\operatorname{cases}^{6}$, but we shall systematically choose this option in the rest of this section. We emphasize that $a$ and $\varphi_{1}\left(x_{1}\right)$ are distinct elements in $I_{0,1}$, yet they are equivalent w.r.t. $\sim_{0,1}$.

The relation $\succsim_{0,1}$ just defined has the following properties.

\section{Lemma 14}

$\succsim_{0,1}$ is a preorder on $I_{0,1}$ which extends $\succsim$ on $I_{0}$, satisfies $\varphi_{1}(x) \succ_{0,1} \varphi_{1}(y)$ for all $x, y \in I_{1}$ with $x \succ y$, and is $d$-separable using $E_{0,1}$.

\footnotetext{
${ }^{6}$ In case $\left(x_{1}, a\right)$ is a hollow (with $x_{1} \in I_{1}$ and $a \in I_{0}$ ), we may decide to set $a \succ_{0,1} \varphi_{1}\left(x_{1}\right)$ instead of $\varphi_{1}\left(x_{1}\right) \sim_{0,1} a$. In such a case, $a$ and $x_{1}$ have to be added to $E_{0,1}$. The latter option can be taken as long as it preserves the denumerable character of the separating set, i.e., for at most a denumerable set of hollows $\left(x_{1}, a\right)$.
} 


\section{PROOF}

The fact that relation $\succsim_{0,1}$ is complete and transitive is an easy consequence of the corresponding properties of $\succsim$ and of the construction of $I_{0,1}$. By construction, we have that $\succsim_{0,1}$ is an extension of $\succsim_{0} I_{0}$, i.e., for all $a, b \in I_{0}, a \succsim_{0,1} b$ iff $a \succsim b$ and that the bijection $\varphi_{1}$ mapping $I_{1}$ onto $\varphi_{1}\left(I_{1}\right)$ respects the order induced by $\succsim$ on $I_{1}$, i.e., for all $x, y \in I_{1}, x \succ y$ iff $\varphi_{1}(x) \succ_{0,1} \varphi_{1}(y)$. The fact that relation $\succsim_{0,1}$ is complete and transitive is an easy consequence of the corresponding properties of $\succsim$ and of the construction of $I_{0,1}$.

The relation $\succsim_{0,1}$ is $d$-separable. Let $z_{1} \succ_{0,1} z_{2}$ with $z_{1}, z_{2} \in I_{0,1}$. Four cases can be distinguished.

Case 1 If $z_{1}, z_{2} \in I_{0}$ then we have $z_{1} \succ_{0,1} z_{2}$ iff $z_{1} \succ z_{2}$. These $z_{1}$ and $z_{2}$ can be separated by an element from $E_{0}$.

Case 2 If $z_{1}=\varphi_{1}\left(x_{1}\right)$ and $z_{2}=\varphi_{1}\left(x_{2}\right)$ for some $x_{1}, x_{2} \in I_{1}$, we know that $z_{1} \succ_{0,1} z_{2}$ implies $x_{1} \succ x_{2}$. Since $\succsim$ is $d$-separable, there is an element in $E_{1}$ separating $x_{1}$ from $x_{2}$. Therefore, $z_{1}$ and $z_{2}$ are separated by an element in $\varphi_{1}\left(E_{1}\right)$.

Case 3 If $z_{1}=\varphi_{1}\left(x_{1}\right)$ and $z_{2} \in I_{0}$, we have that $z_{1} \succ_{0,1} z_{2}$ implies $z_{2} \in B_{x_{1}}^{0}$. If $z_{2}$ is not the greatest element in $B_{x_{1}}^{0}$ (w.r.t. $\succ$ ), then there is $z \in B_{x_{1}}^{0}$ such that $z_{1} \succ_{0,1} z \succ z_{2}$. By hypothesis, $z_{2}$ can be separated from $z$, and therefore from $z_{1}$ by an element from $E_{0}$. If $z_{2}$ is the greatest element in $B_{x_{1}}^{0}$ and there is $x \in J\left(x_{1}\right)$ with $x_{1} \succ x$, then $\varphi_{k}\left(x_{1}\right) \succ_{0,1} \varphi_{k}(x) \succ_{0,1} z_{2}$ and we can use the separability of $\succsim$. Therefore, $z_{1}$ and $z_{2}$ are separated by an element in $\varphi_{1}\left(E_{1}\right)$. Finally, we consider the case in which $z_{2}$ is the greatest element in $B_{x_{1}}^{0}$ and $x_{1}$ is the least element in $J\left(x_{1}\right)$. In such a case, $\left(x_{1}, z_{2}\right)$ is a nose. Since $z_{2}$ is a nose endpoint, it belongs to $E_{0}$ and it separates $z_{1}$ from $z_{2}$.

Case 4 If $z_{1} \in I_{0}$ and $z_{2}=\varphi_{1}\left(x_{2}\right)$, we have that $z_{1} \succ_{0,1} z_{2}$ implies that $z_{1} \in A_{x_{2}}^{0}$. If $z_{1}$ is not the least element in $A_{x_{2}}^{0}$ (w.r.t. $\succsim$ ), then there is $z \in A_{x_{2}}^{0}$ such that $z_{1} \succ z \succ_{0,1} z_{2}$. By hypothesis, $z_{1}$ can be separated from $z$, and therefore from $z_{2}$, by an element from $E_{0}$. If $z_{1}$ is the least element in $A_{x_{2}}^{0}$ and there is $x \in J\left(x_{2}\right)$ such that $x \succ x_{2}$, then $z_{1} \succsim_{0,1} \varphi_{1}(x) \succ_{0,1} \varphi_{1}\left(x_{2}\right)$. Using the separability of $\succsim$, we can separate $z_{1}$ and $z_{2}$ by an element in $\varphi_{1}\left(E_{1}\right)$. Finally, the case in which $z_{1}$ is the least element in $A_{x_{2}}^{0}$ and $x_{2}$ is the greatest element such that $z_{1} \succsim_{0,1} \varphi_{1}\left(x_{2}\right)$ does not occur since this would mean that $\left(x_{2}, z_{1}\right)$ is a hollow; in such a case, by construction, $\varphi_{1}\left(x_{2}\right) \sim_{0,1} z_{1}$ (note that, in the next steps, this situation will not necessarily occur for hollows). 


\subsection{Construction of $I_{0, k}$}

We assume that $I_{0, k-1}$ has been constructed as well as the complete preorder $\succsim_{0, k-1}$ on it. The set $I_{0, k}$ is the union of $I_{0, k-1}$ and the set of the ghosts of the elements of $I_{k}$. The ghost $\varphi_{k}(x)$ for $x \in I_{k}$ is an element which is distinct from all elements of $I_{0, k-1}$. It is defined as being positioned in between the classes of the bipartition $\left(A_{x}, B_{x}\right)$ of the set $I_{0, k-1}$, endowed with the complete preorder $\succsim_{0, k-1}$, with $A_{x} \supseteq \varphi_{k-1}\left(A_{x}^{k-1}\right)$ and $B_{x} \supseteq \varphi_{k-1}\left(B_{x}^{k-1}\right)$.

There is some arbitrariness in the definition of the bipartition $\left(A_{x}, B_{x}\right)$, since there may be elements of $I_{0, k-2}$ lying in between $\varphi_{k-1}\left(A_{x}^{k-1}\right)$ and $\varphi_{k-1}\left(B_{x}^{k-1}\right)$. We can choose to define the bipartition in different ways, for instance, we may assign to $B_{x}$ all the elements of $I_{0, k-2}$ lying in between $\varphi_{k-1}\left(A_{x}^{k-1}\right)$ and $\varphi_{k-1}\left(B_{x}^{k-1}\right)$. Instead, we may assign them all to $A_{x}$.

In the rest of this section, we select one of the possible options and show that it leads to the definition of a $d$-separable complete preorder on $I_{0, k}$.

For $x \in I_{k}$, let now $J(x)$ denote the set of elements of $I_{k}$ determining the same bipartition $\left(A_{x}^{k-1}, B_{x}^{k-1}\right)$ in $I_{k-1}$ as $x$. Let $L(x)$ be the set of elements ${ }^{7}$ of $I_{0, k-1}$ which lie between $\varphi_{k-1}\left(A_{x}^{k-1}\right)$ and $\varphi_{k-1}\left(B_{x}^{k-1}\right)$, i.e., $y \in L(x) \Leftrightarrow a \succ_{0, k-1} y \succ_{0, k-1}$ $b$, for all $a \in \varphi_{k-1}\left(A_{x}^{k-1}\right)$ and $b \in \varphi_{k-1}\left(B_{x}^{k-1}\right)$. We define the bipartition $\left(A_{x}, B_{x}\right)$ as follows:

$$
\begin{aligned}
& A_{x}=L(x) \cup\left\{z \in I_{0, k-1}: \exists a \in \varphi_{k-1}\left(A_{x}^{k-1}\right) \text { such that } z \succsim_{0, k-1} a\right\}, \\
& B_{x}=I_{0, k-1} \backslash A_{x}=\left\{y \in I_{0, k-1}: \exists b \in \varphi_{k-1}\left(B_{x}^{k-1}\right) \text { such that } b \succsim_{0, k-1} y\right\} .
\end{aligned}
$$

We insert ghosts as follows. We set $a \succsim_{0, k} \varphi_{k}\left(x^{\prime}\right) \succsim_{0, k} \varphi_{k}\left(x^{\prime \prime}\right) \succ_{0, k} b$, for all $a \in A_{x}, b \in B_{x}$ and $x^{\prime}, x^{\prime \prime} \in J(x)$ with $x^{\prime} \succsim x^{\prime \prime}$. We also impose $\varphi_{k}\left(x^{\prime}\right) \succ_{0, k} \varphi_{k}\left(x^{\prime \prime}\right)$ whenever $x^{\prime} \succ x^{\prime \prime}$ (see Figure 2). In the particular case in which $A_{x}$ has a least element $\underline{a}$ (w.r.t. $\left.\succsim_{0, k-1}\right)$ and $J(x)$ has a greatest element $\bar{x}$ (w.r.t. $\succsim$ ), we set $^{8}$ $\varphi_{k}(\bar{x}) \sim_{0, k}$ a. The relation $\succsim_{0, k}$ on $I_{0, k}$ is completely defined as follows. For all $z_{1}, z_{2} \in I_{0, k}$, we have the following cases:

Case 1 if $z_{1}, z_{2} \in I_{0, k-1}$, we have $z_{1} \succsim_{0, k} z_{2}$ iff $z_{1} \succsim_{0, k-1} z_{2}$, hence $\succsim_{0, k}$ extends $\succsim_{0, k-1}$;

Case 2 if $z_{1}=\varphi_{k}\left(x_{1}\right)$ and $z_{2}=\varphi_{k}\left(x_{2}\right)$ for some $x_{1}, x_{2} \in I_{k}$, we set $z_{1} \succ_{0, k} z_{2}$ iff $x_{1} \succ x_{2}$; note that this definition is compatible with the construction above also in case $x_{1}$ and $x_{2}$ determine the same bipartition in $I_{k-1}$;

\footnotetext{
${ }^{7}$ Actually, $L(x)$ is empty in case $k=1$ and $L(x) \subseteq I_{0, k-2}$ if $k \geq 2$.

${ }^{8}$ Note that $\underline{a}$ is not necessarily the ghost of an element $y \in I_{k-1}$ such that $(\bar{x}, y)$ is a hollow. It can happen that a least element in $A_{x}$ is $\underline{a} \in L(x)$, which is the ghost of an element of $I_{l}$, for $0 \leq l<k-1$ (for more on this case, see Section 4.9).
} 
Case 3 if $z_{1}=\varphi_{k}\left(x_{1}\right)$ and $z_{2} \in I_{0, k-1}$, we have $z_{1} \succ_{0, k} \quad z_{2}$ iff $z_{2} \in B_{x_{1}}$ and $z_{2} \succsim_{0, k} z_{1}$ iff $z_{2} \in A_{x_{1}}$. Furthermore, $z_{2} \sim_{0, k} z_{1}$ iff $z_{2}$ is a least element in $A_{x_{1}}$ (w.r.t. $\left.\succsim_{0, k-1}\right)$ and $x_{1}$ is the greatest element in $J\left(x_{1}\right)$ (w.r.t. $\succsim$ ).

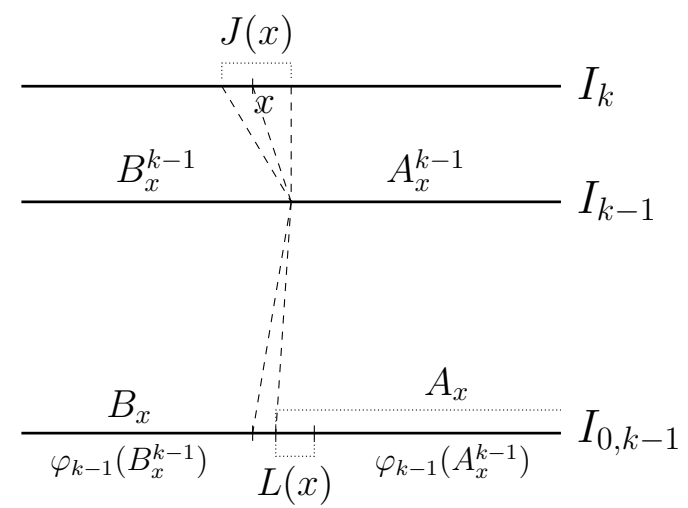

Figure 2: Ghost insertion for the elements of $I_{k}$

With this definition, we have the following result, which generalizes Lemma 14.

\section{Lemma 15}

$\succsim_{0, k}$ is a complete preorder on $I_{0, k}$ which extends $\succsim_{0, k-1}$, satisfies $\varphi_{k}(x) \succsim_{0, k} \varphi_{k}(y)$ for all $x, y \in I_{k}$ with $x \succsim y$, and is d-separable by $E_{0, k}$.

\section{ProOF}

It is easy to check that the relation $\succsim_{0, k}$ defined above is complete, transitive, extends $\succsim_{0, k-1}$. It reproduces on $\varphi_{k}\left(I_{k}\right)$ the order induced by the trace $\succsim$ on $I_{k}$.

We prove that relation $\succsim_{0, k}$ is $d$-separable. We know by Lemma 14 that $\succsim_{0,1}$ is $d$-separated by $E_{0,1}$. We prove the result by induction, assuming that $\succsim_{0, k-1}$ is $d$-separated by $E_{0, k-1}$. Let $z_{1}, z_{2} \in I_{0, k}$ be such that $z_{1} \succ_{0, k} z_{2}$.

Case 1 If $z_{1}, z_{2} \in I_{0, k-1}$ and $z_{1} \succ_{0, k} z_{2}$, we have $z_{1} \succ_{0, k-1} z_{2}$. By the induction hypothesis, we know that $z_{1}$ and $z_{2}$ can be separated by an element from $E_{0, k-1}$.

Case 2 If $z_{1}=\varphi_{k}\left(x_{1}\right)$ and $z_{2}=\varphi_{k}\left(x_{2}\right)$ for some $x_{1}, x_{2} \in I_{k}$, we know that $z_{1} \succ_{0, k} z_{2}$ implies $x_{1} \succ x_{2}$. Since $\succsim$ is $d$-separable, there is an element in $E_{k}$ separating $x_{1}$ from $x_{2}$. Therefore, $z_{1}$ and $z_{2}$ are separated by an element in $\varphi_{k}\left(E_{k}\right)$.

Case 3 If $z_{1}=\varphi_{k}\left(x_{1}\right)$ and $z_{2} \in I_{0, k-1}$, we have that $z_{1} \succ_{0, k} z_{2}$ implies that $z_{2} \in B_{x_{1}}$. If $z_{2}$ is not a greatest element in $B_{x_{1}}$ (w.r.t. $\succsim_{0, k-1}$ ), then there 
is $z \in B_{x_{1}}$ such that $z_{1} \succ_{0, k} z \succ_{0, k-1} z_{2}$. By the induction hypothesis, $z_{2}$ can be separated from $z$, and therefore from $z_{1}$, by an element from $E_{0, k-1}$. If $z_{2}$ is a greatest element in $B_{x_{1}}$ and there is $x \in J\left(x_{1}\right)$ such that $x_{1} \succ x$, then $\varphi_{k}\left(x_{1}\right) \succ_{0, k} \varphi_{k}(x) \succ_{0, k} z_{2}$ and we can use the separability of $\succsim$. Therefore, $z_{1}$ and $z_{2}$ are separated by an element in $\varphi_{k}\left(E_{k}\right)$. Finally, we have to consider the case in which $z_{2}$ is a greatest element in $B_{x_{1}}$ and $x_{1}$ is the least element in $J\left(x_{1}\right)$. In this case, there is $y \in I_{k-1}$ such that $\left(x_{1}, y\right)$ is a nose and $\varphi_{k-1}(y) \sim_{k-1} z_{2}$. Since $y$ is a nose endpoint, it belongs to $E_{k-1}$ and $\varphi_{k-1}(y) \in E_{0, k}$ separates $z_{1}$ from $z_{2}$.

Case 4 If $z_{1} \in I_{0, k-1}$ and $z_{2}=\varphi_{k}\left(x_{2}\right)$, we have that $z_{1} \succ_{0, k} z_{2}$ implies that $z_{1} \in A_{x_{2}}$. If $z_{1}$ is not the least element in $A_{x_{2}}$ (w.r.t. $\succsim_{0, k-1}$ ), then there is $z \in A_{x_{2}}$ such that $z_{1} \succ_{0, k} z \succ_{0, k-1} z_{2}$. By the induction hypothesis, $z_{1}$ can be separated from $z$, and therefore from $z_{1}$, by an element from $E_{0, k-1}$. If $z_{1}$ is a least element in $A_{x_{2}}$ and there is $x \in J\left(x_{2}\right)$ with $x \succ x_{2}$, then $z_{1} \succ_{0, k} \varphi_{k}(x) \succ_{0, k} \varphi_{k}\left(x_{2}\right)$, and we can use the separability of $\succsim$. Therefore, $z_{1}$ and $z_{2}$ are separated by an element in $\varphi_{k}\left(E_{k}\right)$. Finally, we have to consider the case in which $z_{1}$ is a least element in $A_{x_{2}}$ and $x_{2}$ is the greatest element in $J\left(x_{2}\right)$. This is incompatible with $z_{1} \succ_{0, k} z_{2}$. Indeed, we decided to place $z_{2}=\varphi_{k}\left(x_{2}\right)$ in the equivalence class of $z_{1}$. We thus have $z_{2} \sim_{k} z_{1}$, which contradicts $z_{1} \succ_{0, k} z_{2}$.

\subsection{Construction of $I_{-1, k}$}

We assume that $I_{0, k}$ has been constructed as well as the complete preorder $\succsim_{0, k}$ on it. The set $I_{-1, k}$ is the union of $I_{0, k}$ and the set of the ghosts of the elements in $I_{-1}$. The ghost $\varphi_{-1}(x)$ for $x \in I_{-1}$ is an element which is distinct from all elements in $I_{0, k}$. It is defined as being positioned in between the classes of a bipartition $\left(C_{x}, D_{x}\right)$ of the set $I_{0, k}$, endowed with the complete preorder $\succsim_{0, k}$, with $C_{x} \supseteq C_{x}^{0}$ and $D_{x} \supseteq D_{x}^{0}$. There is some arbitrariness in the definition of the bipartition $\left(C_{x}, D_{x}\right)$, since there may be elements of $I_{0, k}$ lying in between $C_{x}^{0}$ and $D_{x}^{0}$.

We select one of the possible options and show that it leads to the definition of a $d$-separable complete preorder on $I_{-1, k}$. For $x \in I_{-1}$, let now $K(x)$ denote the set of elements of $I_{-1}$ determining the same bipartition $\left(C_{x}^{0}, D_{x}^{0}\right)$ in $I_{0}$ as $x$. Let $M(x)$ be the set of elements in $I_{0, k}$ which lie between $C_{x}^{0}$ and $D_{x}^{0}$, i.e., $y \in M(x) \Leftrightarrow c \succ_{0, k} y \succ_{0, k} d$, for all $c \in C_{x}^{0}$ and $d \in D_{x}^{0}$. We define the bipartition $\left(C_{x}, D_{x}\right)$ as follows:

$$
\begin{aligned}
& C_{x}=\left\{z \in I_{0, k}: \exists c \in C_{x}^{0} \text { such that } z \succsim_{0, k} c\right\}, \\
& D_{x}=I_{0, k} \backslash C_{x}=M(x) \cup\left\{y \in I_{0, k}: \exists d \in D_{x}^{0} \text { such that } d \succsim_{0, k} y\right\} .
\end{aligned}
$$


We insert ghosts as follows. We set $c \succsim_{-1, k} \varphi_{-1}\left(x^{\prime}\right) \succsim_{-1, k} \varphi_{-1}\left(x^{\prime \prime}\right) \succsim_{-1, k} d$, for all $c \in C_{x}, d \in D_{x}$ and $x^{\prime}, x^{\prime \prime} \in K(x)$ with $x^{\prime} \succsim x^{\prime \prime}$. We also impose $\varphi_{-1}\left(x^{\prime}\right) \succ_{-1, k}$ $\varphi_{-1}\left(x^{\prime \prime}\right)$ whenever $x^{\prime} \succ x^{\prime \prime}$ (see Figure 3 ). In the particular case in which $D_{x}$ has a greatest element $\bar{d}$ (w.r.t. $\succsim_{0, k}$ ) and $K(x)$ has a least element $\underline{x}$ (w.r.t. $\succsim$ ), we $\operatorname{set}^{9} \varphi_{-1}(\underline{x}) \sim_{-1, k} \bar{d}$. The relation $\succsim_{-1, k}$ on $I_{-1, k}$ is completely defined as follows. For all $z_{1}, z_{2} \in I_{-1, k}$, we have the following cases:

Case 1 if $z_{1}, z_{2} \in I_{0, k}$, we have $z_{1} \succsim_{-1, k} z_{2}$ iff $z_{1} \succsim_{0, k} z_{2}$, hence $\succsim_{-1, k}$ extends $\succsim_{0, k}$;

Case 2 if $z_{1}=\varphi_{-1}\left(x_{1}\right)$ and $z_{2}=\varphi_{-1}\left(x_{2}\right)$ for some $x_{1}, x_{2} \in I_{-1}$, we set $z_{1} \succ_{-1, k} z_{2}$ iff $x_{1} \succ x_{2}$; note that this definition is compatible with the construction above also in case $x_{1}$ and $x_{2}$ determine the same bipartition in $I_{0}$;

Case 3 if $z_{1}=\varphi_{-1}\left(x_{1}\right)$ and $z_{2} \in I_{0, k}$, we have $z_{1} \succsim_{-1, k} z_{2}$ iff $z_{2} \in D_{x_{1}}$, and $z_{2} \succ_{-1, k} z_{1}$ iff $z_{2} \in C_{x_{1}}$. Furthermore, $z_{2} \sim_{-1, k} z_{1}$ iff $z_{2}$ is a greatest element in $D_{x_{1}}$ (w.r.t. $\left.\succsim_{0, k}\right)$ and $x_{1}$ is the least element in $K\left(x_{1}\right)$ (w.r.t. $\left.\succsim\right)$.

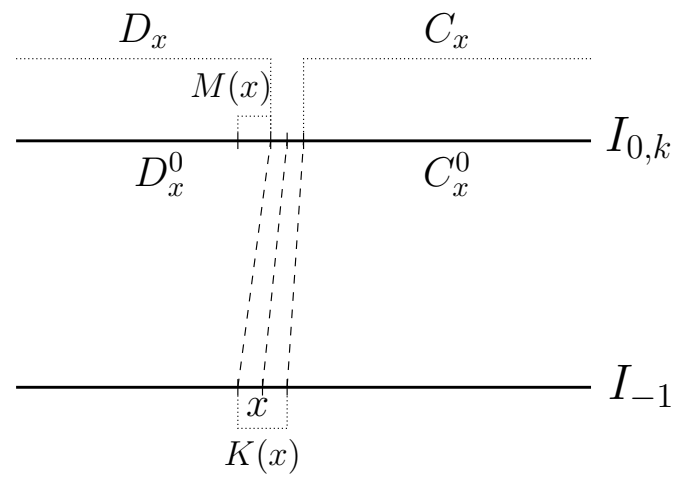

Figure 3: Ghost insertion for $I_{-1}$ into $I_{0, k}$

With this definition, the following lemma, which extends Lemma 15, holds.

\section{Lemma 16}

$\succsim_{-1, k}$ is a complete preorder on $I_{-1, k}$ which extends $\succsim_{0, k}$, satisfies $\varphi_{k}(x) \succsim_{-1, k}$ $\varphi_{k}(y)$ for all $x, y \in I_{-1}$ with $x \succsim y$, and is d-separable by $E_{-1, k}$.

\section{PROOF}

It is easy to check that the relation $\succsim_{-1, k}$ defined above is complete, transitive and extends $\succsim_{0, k}$. It reproduces on $\varphi_{k}\left(I_{-1}\right)$ the order induced by the trace $\succsim$ on $I_{-1}$. We prove that relation $\succsim_{-1, k}$ is $d$-separable. We know by Lemma 15 that $\succsim_{0, k}$ is $d$-separated by $E_{0, k}$. Let $z_{1}, z_{2} \in I_{-1, k}$ be such that $z_{1} \succ_{-1, k} z_{2}$.

\footnotetext{
${ }^{9}$ Note that $(\bar{d}, \underline{x})$ is a hollow only if $\bar{d} \in I_{0}$. Actually, $\bar{d}$ could belong to $M(x)$ and be the ghost of an element in $I_{l}$, for $1 \leq l \leq k$. In such a case $(\bar{d}, \underline{x})$ is no hollow.
} 
Case 1 If $z_{1}, z_{2} \in I_{0, k}$ and $z_{1} \succ_{-1, k} z_{2}$, we have $z_{1} \succ_{0, k} z_{2}$. By Lemma 15, we know that $z_{1}$ and $z_{2}$ can be separated by an element from $E_{0, k}$.

Case 2 If $z_{1}=\varphi_{-1}\left(x_{1}\right)$ and $z_{2}=\varphi_{-1}\left(x_{2}\right)$ for some $x_{1}, x_{2} \in I_{-1}$, we know that $z_{1} \succ_{-1, k} z_{2}$ implies $x_{1} \succ x_{2}$. Since $\succsim$ is $d$-separable, there is an element in $E_{-1}$ separating $x_{1}$ from $x_{2}$. Therefore, $z_{1}$ and $z_{2}$ are separated by an element in $\varphi_{-1}\left(E_{-1}\right)$.

Case 3 If $z_{1}=\varphi_{-1}\left(x_{1}\right)$ and $z_{2} \in I_{0, k}$, we have that $z_{1} \succ_{-1, k} z_{2}$ implies that $z_{2} \in D_{x_{1}}$. If $z_{2}$ is not a greatest element in $D_{x_{1}}$ (w.r.t. $\succsim_{0, k}$ ), then there is $z \in D_{x_{1}}$ such that $z_{1} \succ_{-1, k} z \succ_{0, k} z_{2}$. By Lemma $15, z_{2}$ can be separated from $z$, and therefore from $z_{1}$, by an element from $E_{0, k}$. If $z_{2}$ is a greatest element in $D_{x_{1}}$ and there is $x \in M\left(x_{1}\right)$ such that $x_{1} \succ x$, then $\varphi_{-1}\left(x_{1}\right) \succ_{-1, k}$ $\varphi_{-1}(x) \succ_{-1, k} z_{2}$, and we can use the separability of $\succsim$. Therefore, $z_{1}$ and $z_{2}$ are separated by an element in $\varphi_{-1}\left(E_{-1}\right)$. Finally, we have to consider the case in which $z_{2}$ is a greatest element in $D_{x_{1}}$ and $x_{1}$ is the least element in $K\left(x_{1}\right)$. This is incompatible with $z_{1} \succ_{-1, k} z_{2}$ because, in this case, we have decided that $z_{1}=\varphi_{-1}\left(x_{1}\right) \sim_{-1, k} z_{2}$.

Case 4 If $z_{1} \in I_{0, k}$ and $z_{2}=\varphi_{-1}\left(x_{2}\right)$, we have that $z_{1} \succ_{0, k} z_{2}$ implies $z_{1} \in C_{x_{2}}$. If $z_{1}$ is not a least element in $C_{x_{2}}$ (w.r.t. $\succsim_{0, k}$ ), then there is $z \in C_{x_{2}}$ such that $z_{1} \succ_{-1, k} z \succ_{0, k} z_{2}$. By Lemma $15, z_{1}$ can be separated from $z$, and therefore from $z_{2}$, by an element from $E_{0, k}$. If $z_{1}$ is a least element in $C_{x_{2}}$ and there is $x \in I_{-1}$ with $z_{2} \succ_{0, k} \varphi_{-1}(x) \succ_{0, k} \varphi_{-1}\left(x_{2}\right)$, then we can use the separability of $\succsim$. Therefore, $z_{1}$ and $z_{2}$ are separated by an element in $\varphi_{-1}\left(E_{-1}\right)$. Finally, we have to consider the case in which $z_{1}$ is a least element in $C_{x_{2}}$ and $x_{2}$ is the greatest element in $K\left(x_{2}\right)$. In this case, there is $y \in I_{0}$ such that $\left(y, x_{2}\right)$ is a nose and $y \sim_{0, k} z_{1}$. Since $y$ is a nose endpoint, it belongs to $E_{0}$ and it separates $z_{1}$ from $z_{2}$.

\subsection{Construction of $I_{-l, k}$}

This is the general step of the construction. Actually, depending on the order in which we perform the ghosts insertions, we may need to build $I_{-l, k}$ starting either from $I_{-l+1, k}$ or from $I_{-l, k-1}$. We describe the construction of $I_{-l, k}$ starting from $I_{-l+1, k}$, leaving the construction starting from $I_{-l, k-1}$ to the reader.

We assume that $I_{-l+1, k}$ has been constructed as well as the complete preorder $\succsim_{-l+1, k}$ on it. The set $I_{-l, k}$ is the union of $I_{-l+1, k}$ and the set of the ghosts of the elements in $I_{-l}$. The ghost $\varphi_{-l}(x)$ for $x \in I_{-l}$ is an element which is distinct from all elements of $I_{-l+1, k}$. It is defined as being positioned in between the classes of a bipartition $\left(C_{x}, D_{x}\right)$ of the set $I_{-l+1, k}$, endowed with the complete preorder $\succsim_{-l+1, k}$, 
with $C_{x} \supseteq \varphi_{-l+1}\left(C_{x}^{-l+1}\right)$ and $D_{x} \supseteq \varphi_{-l+1}\left(D_{x}^{-l+1}\right)$. There is some arbitrariness in the definition of the bipartition $\left(C_{x}, D_{x}\right)$, since there may be elements of $I_{-l+1, k}$ lying in between $\varphi_{-l+1}\left(C_{x}^{-l+1}\right)$ and $\varphi_{-l+1}\left(D_{x}^{-l+1}\right)$.

We select one of the possible options and show that it leads to the definition of a $d$-separable complete preorder on $I_{-l, k}$. For $x \in I_{-l}$, let $K(x)$ denote the set of elements of $I_{-l}$ determining the same bipartition $\left(C_{x}^{-l+1}, D_{x}^{-l+1}\right)$ in $I_{-l+1}$ as $x$. Let $M(x)$ be the set of elements of $I_{-l+1, k}$ which lie between $\varphi_{-l+1}\left(C_{x}^{-l+1}\right)$ and $\varphi_{-l+1}\left(D_{x}^{-l+1}\right)$, i.e., $y \in M(x) \Leftrightarrow c \succ_{0, k} y \succ_{0, k} d$, for all $c \in \varphi_{-l+1}\left(C_{x}^{-l+1}\right)$ and $d \in \varphi_{-l+1}\left(D_{x}^{-l+1}\right)$. We define the bipartition $\left(C_{x}, D_{x}\right)$ as follows:

$C_{x}=\left\{z \in I_{-l+1, k}: \exists c \in \varphi_{-l+1}\left(C_{x}^{-l+1}\right)\right.$ such that $\left.z \succsim_{-l+1, k} c\right\}$,

$D_{x}=I_{-l+1, k} \backslash C_{x}=M(x) \cup\left\{y \in I_{-l+1, k}: \exists d \in \varphi_{-l+1}\left(D_{x}^{-l+1}\right)\right.$ such that $\left.d \succsim_{-l+1, k} y\right\}$.

We insert ghosts as follows. We set $c \succsim_{-l, k} \varphi_{-l}\left(x^{\prime}\right) \succsim_{-l, k} \varphi_{-1}\left(x^{\prime \prime}\right) \succsim_{-l, k} d$, for all $c \in C_{x}, d \in D_{x}$ and $x^{\prime}, x^{\prime \prime} \in K(x)$ with $x^{\prime} \succsim x^{\prime \prime}$. We also impose $\varphi_{-l}\left(x^{\prime}\right) \succ_{-l, k}$ $\varphi_{-l}\left(x^{\prime \prime}\right)$ whenever $x^{\prime} \succ x^{\prime \prime}$ (see Figure 4 ). In the particular case in which $D_{x}$ has a greatest element $\bar{d}$ (w.r.t. $\succsim_{-l+1, k}$ ) and $K(x)$ has a least element $\underline{x}$ (w.r.t. $\succsim$ ), we $\operatorname{set}^{10} \varphi_{-l}(\underline{x}) \sim_{-l, k} \bar{d}$. The relation $\succsim_{-l, k}$ on $I_{-l, k}$ is completely defined as follows. For all $z_{1}, z_{2} \in I_{-l, k}$, we distinguish the following cases:

Case 1 if $z_{1}, z_{2} \in I_{-l+1, k}$, we have $z_{1} \succsim_{-l, k} z_{2}$ iff $z_{1} \succsim_{-l+1, k} z_{2}$, hence $\succsim_{-1, k}$ extends $\succsim-l+1, k$;

Case 2 if $z_{1}=\varphi_{-l}\left(x_{1}\right)$ and $z_{2}=\varphi_{-l}\left(x_{2}\right)$ for some $x_{1}, x_{2} \in I_{-l}$, we set $z_{1} \succ_{-l, k} z_{2}$ iff $x_{1} \succ x_{2}$; note that this definition is compatible with the construction above also in case $x_{1}$ and $x_{2}$ determine the same bipartition in $I_{-l+1}$;

Case 3 if $z_{1}=\varphi_{-l}\left(x_{1}\right)$ and $z_{2} \in I_{-l+1, k}$, we have $z_{1} \succsim_{-l, k} z_{2}$ iff $z_{2} \in D_{x_{1}}$, and $z_{2} \succ_{-l, k} z_{1}$ iff $z_{2} \in C_{x_{1}}$. Furthermore, $z_{2} \sim_{-l, k} z_{1}$ iff $z_{2}$ is a greatest element in $D_{x_{1}}$ (w.r.t. $\succsim_{-l+1, k}$ ) and $x_{1}$ is the least element in $K\left(x_{1}\right)$ (w.r.t. $\succsim$ ).

With this definition, the following lemma, which extends Lemma 16, holds.

\section{Lemma 17}

$\succsim_{-l, k}$ is a complete preorder on $I_{-l, k}$ which extends $\succsim_{-l+1, k}$, satisfies $\varphi_{k}(x) \succsim_{-l, k}$ $\varphi_{k}(y)$ for all $x, y \in I_{-l}$ with $x \succsim y$, and is d-separable by $E_{-l, k}$.

\section{ProOF}

It is easy to check that the relation $\succsim_{-l, k}$ defined above is complete, transitive and extends $\succsim_{-l+1, k}$. It reproduces on $\varphi_{k}\left(I_{-l}\right)$ the order induced by the trace $\succsim$ on $I_{-l}$.

\footnotetext{
${ }^{10}$ The pair $(\bar{d}, \underline{x})$ is a hollow if and only if $\bar{d}$ is the ghost of an element of $I_{-l+1}$. This is not always the case since $\bar{d}$ can belong to $M(x)$ (see also footnote 9 ).
} 


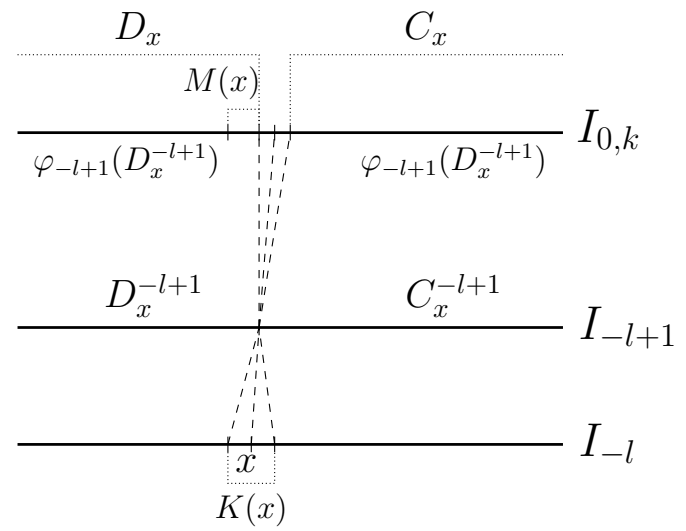

Figure 4: Ghost insertion for $I_{-l}$ into $I_{-l+1, k}$

We prove that relation $\succsim_{-l, k}$ is $d$-separable. We know by Lemma 16 that $\succsim_{-1, k}$ is $d$-separated by $E_{-1, k}$. We prove the result by induction, assuming that $\succsim_{-l+1, k}$ is $d$-separable by $E_{-l+1, k}$. Let $z_{1}, z_{2} \in I_{-l, k}$ be such that $z_{1} \succ_{-l, k} z_{2}$.

Case 1 If $z_{1}, z_{2} \in I_{-l+1, k}$ and $z_{1} \succ_{-l, k} z_{2}$, we have $z_{1} \succ_{-l+1, k} z_{2}$. By the induction hypothesis, $z_{1}$ and $z_{2}$ can be separated by an element from $E_{-l+1, k}$.

Case 2 If $z_{1}=\varphi_{-l}\left(x_{1}\right)$ and $z_{2}=\varphi_{-l}\left(x_{2}\right)$ for some $x_{1}, x_{2} \in I_{-l}$, we know that $z_{1} \succ_{-l, k} z_{2}$ implies $x_{1} \succ x_{2}$. Since $\succsim$ is $d$-separable, there is an element in $E_{-l}$ separating $x_{1}$ from $x_{2}$. Therefore, $z_{1}$ and $z_{2}$ are separated by an element in $\varphi_{-l}\left(E_{-l}\right)$.

Case 3 If $z_{1}=\varphi_{-l}\left(x_{1}\right)$ and $z_{2} \in I_{-l+1, k}$, we have that $z_{1} \succ_{-l, k} z_{2}$ implies that $z_{2} \in D_{x_{1}}$. If $z_{2}$ is not a greatest element in $D_{x_{1}}$ (w.r.t. $\succsim_{-l+1, k}$ ), then there is $z \in D_{x_{1}}$ such that $z_{1} \succ_{-l, k} z \succ_{-l+1, k} z_{2}$. By Lemma $15, z_{2}$ can be separated from $z$, and therefore from $z_{1}$, by an element from $E_{-l+1, k}$. If $z_{2}$ is a greatest element in $D_{x_{1}}$ and there is $x \in M\left(x_{1}\right)$ such that $x_{1} \succ x$, then $\varphi_{-l}\left(x_{1}\right) \succ_{-l, k} \varphi_{-1}(x) \succ_{-l, k} z_{2}$, and we can use the separability of $\succsim$. Therefore, $z_{1}$ and $z_{2}$ are separated by an element in $\varphi_{-l}\left(E_{-l}\right)$. Finally, we have to consider the case in which $z_{2}$ is a greatest element in $D_{x_{1}}$ and $x_{1}$ is the least element in $K\left(x_{1}\right)$. This is incompatible with $z_{1} \succ_{-l, k} z_{2}$ because, in this case, we decided that $z_{1}=\varphi_{-l}\left(x_{1}\right) \sim_{-l, k} z_{2}$.

Case 4 If $z_{1} \in I_{-l+1, k}$ and $z_{2}=\varphi_{-l}\left(x_{2}\right)$ for some $x_{2} \in I_{-l}$, we have that $z_{1} \succ_{-l, k} z_{2}$ implies $z_{1} \in C_{x_{2}}$. If $z_{1}$ is not a least element in $C_{x_{2}}$ (w.r.t. $\succsim-l+1, k$ ), then there is $z \in C_{x_{2}}$ such that $z_{1} \succ_{-l+1, k} z \succ_{-l, k} z_{2}$. By the induction hypothesis, $z_{1}$ can be separated from $z$, and therefore from $z_{2}$, by an element from $E_{-l+1, k}$. If $z_{1}$ is a least element in $C_{x_{2}}$ and there is $x \in K\left(x_{2}\right)$ with $x \succ x_{2}$, 
then $z_{2} \succ_{-l, k} \varphi_{-l}(x) \succ_{-l, k} \varphi_{-l}\left(x_{2}\right)$, and we can use the separability of $\succsim$. Therefore, $z_{1}$ and $z_{2}$ are separated by an element in $\varphi_{-l}\left(E_{-l}\right)$. Finally, we have to consider the case in which $z_{1}$ is a least element in $C_{x_{2}}$ and $x_{2}$ is the greatest element in $K\left(x_{2}\right)$. In this case, there is $y \in I_{0}$ such that $\left(y, x_{2}\right)$ is a nose and $\varphi_{-l+1}(y) \sim_{-l+1, k} z_{1}$. Since $y$ is a nose endpoint, it belongs to $E_{-l+1}$ and $\varphi_{-l+1}(y) \in E_{-l+1, k}$ separates $z_{1}$ from $z_{2}$.

At the end of this construction process, involving at most a countably infinite number of steps, we obtain the set

$$
\tilde{I}_{0}=I_{0} \cup\left(\bigcup_{k \geq 0} \varphi_{k}\left(I_{k}\right)\right) \cup\left(\bigcup_{l>0} \varphi_{-l}\left(I_{-l}\right)\right)=I_{0} \cup\left(\bigcup_{m \in M} \varphi_{m}\left(I_{m}\right)\right),
$$

ordered by $\succsim_{\varphi}$, which is an extension of the linear order $\succsim$ on $I_{0}$. It also extends the complete preorder $\succsim_{-l, k}$, for all $k,-l \in M$. The restriction of $\succsim_{\varphi}$ to $\varphi_{m}\left(I_{m}\right)$, for $m \in M, m \neq 0$, is an isomorphic image of the linear order $\succsim$ on $I_{k}$. The denumerable set $\tilde{E}_{0}=E_{0} \cup\left(\bigcup_{m \in M} \varphi_{m}\left(E_{m}\right)\right) d$-separates the complete preorder $\succsim_{\varphi}$. Indeed, for all $x \neq y \in \tilde{I}_{0}, x$ and $y$ belong to $I_{-l, k}$ for some $-l, k \in M$ and by Lemma 17 , they are separated by some element in $E_{-l, k}$. Note that, while $\succsim$ is a linear order on $X$ (provided no pair of distinct elements are equivalent in the semiorder, an hypothesis that was made w.l.o.g. in Section 2.1), $\succsim_{\varphi}$ is a complete preorder, in general. The equivalence class of an element may not be reduced to a singleton since there are cases in which we set a ghost equivalent to another element or another ghost in the course of the construction of $\left(\tilde{I}_{0}, \succsim_{\varphi}\right)$ (see also Remark 34 in Bouyssou and Pirlot (2020a) for other possible cases of equivalence of ghosts corresponding to elements in consecutive sets $I_{m}$ and $I_{m+1}$ ).

\section{Remark 18}

Starting from $I_{0,1}$ we may construct the ghosts of all the elements of a connected semiorder. In case the decomposition in subsets $\left(\ldots, I_{-l}, \ldots, I_{0}, \ldots, I_{k}, \ldots\right)$ involves finitely many subsets, the construction may proceed by first exhausting all positive indices $k$, i.e., by constructing $I_{0, \bar{k}}$ (using section 4.4) and then building $I_{-l, \bar{k}}$, for $l=1, \ldots, \bar{l}$ (using sections 4.5 and 4.6). In case there are a countably infinite number of subsets in the decomposition, one may proceed by addressing alternatively the positive and the negative labels. For instance: $I_{0,1}, I_{-1,1}, I_{-1,2}, I_{-2,2}, \ldots$. The previous sections allow to do that provided we consider as established the option left to the reader in section 4.6, i.e., building $I_{-l, k}$ from $I_{-l, k-1}$. 


\subsection{Construction of a representation on an $I$-connected component}

Since the complete preorder $\succsim_{\varphi}$ on $\tilde{I}_{0}$ is $d$-separable, there exists a numerical representation $f: \tilde{I}_{0} \rightarrow \mathbb{R}$ of $\succsim_{\varphi}$ such that $f(x) \geq f(y)$, for all $x, y \in \tilde{I}_{0}$ such that $x \succsim_{\varphi} y$.

In order to build a unit representation of the semiorder $S=(P, I)$, we select a numerical representation $f$ of $\succsim_{\varphi}$ into the $] 0,1[$ real interval and we define the function $u$ on $\mathcal{D}$ as follows:

$$
u(x)=f\left(\varphi_{m}(x)\right)+m \text { for all } x \in I_{m},
$$

for all $m \in M$, and interpreting $\varphi_{0}$ as the identity function.

\section{Proposition 19}

If $f: X \rightarrow] 0,1\left[\subset \mathbb{R}\right.$ is a numerical representation of $\succsim_{\varphi}$ on $\tilde{I}_{0}$, then the function $u$ defined by (6) is a unit representation of the semiorder $S=(P, I)$ restricted to $\mathcal{D}$, i.e., for all $x, y \in \mathcal{D}$,

$$
\begin{array}{cll}
u(x)>u(y)+1 & \text { iff } & x P y \\
-1 \leq u(x)-u(y) \leq 1 & \text { iff } & x I y
\end{array}
$$

\section{PROOF}

Let $x, y$ be such that $x P y$. If $y$ belongs to $I_{k}(k \in M)$, we have that $x$ belongs to $I_{m}$ for $m \geq k+1$ (by Proposition 13, items 13.4, 13.5 and 13.6). If $x \in I_{k+1}$, we have $u(x)-u(y)=f\left(\varphi_{k+1}(x)\right)+k+1-f\left(\varphi_{k}(y)\right)-k>1$, since $\varphi_{k+1}(x) \succ_{\varphi} \varphi_{k}(y)$ by construction, and therefore $f\left(\varphi_{k+1}(x)\right)>f\left(\varphi_{k}(y)\right)$.

If $x \in I_{m}$, for $m \geq k+2$, we have $u(x)-u(y)=f\left(\varphi_{m}(x)\right)+m-f\left(\varphi_{k}(y)\right)-k \geq 1$ since $m-k \geq 2$ and $\left|f\left(\varphi_{m}(x)\right)-f\left(\varphi_{k}(y)\right)\right| \leq 1$. Actually, $u(x)-u(y)>1$. Assume for contradiction that $u(x)-u(y)=1$. This implies that $m=k+2$ and $f\left(\varphi_{m}(x)\right)-f\left(\varphi_{k}(y)\right)=-1$. The latter means that $\varphi_{m}(x)$ is the least element in $\left(\tilde{I}_{0}, \succsim_{\varphi}\right)$ and $\varphi_{k}(y)$ is the largest. Consider first the case in which $m>0$. Since $\varphi_{m}(x)$ is the least element in $\tilde{I}_{0}$, we have that $\varphi_{m}(x) \precsim \varphi \varphi_{m-1}(z)$, for all $z \in I_{m-1}$. Therefore, $x I z$, for all $z \in I_{m-1}$. This implies that $x \in I_{m-1}$, by construction of $I_{m-1}$, a contradiction. Turning to the case in which $m \leq 0$, i.e., $k \leq-2$, we deduce similarly that $y I w$, for all $w \in I_{k+1}$. This implies that $y \in I_{k+1}$, a contradiction.

Consider now a pair $x, y \in \mathcal{D}$ such that $x I y$. We assume w.l.o.g. that $x \succ y$ and $y \in I_{k}(k \in M)$. By Proposition 13, items 13.2, 13.3 and 13.5, we know that $x \in I_{k}$ or $x \in I_{k+1}$. In the former case, $0<u(x)-u(y)=f\left(\varphi_{k}(x)\right)+k-f\left(\varphi_{k}(y)\right)-k \leq 1$, since $0 \leq f \leq 1$. In the latter case, we have $0<u(x)-u(y)=f\left(\varphi_{k+1}(x)\right)+k+$ $1-f\left(\varphi_{k}(y)\right)-k<1$ because $f\left(\varphi_{k+1}(x)\right)-f\left(\varphi_{k}(y)\right) \leq 0$. To establish this, we consider the following two possible cases: 
- $k \geq 0$. By construction of the ghosts and the extension $\succsim_{\varphi}$ of $\succsim$ for $k \geq 0$, we have $\varphi_{k}(a) \succsim_{\varphi} \varphi_{k+1}(x) \succ_{\varphi} \varphi_{k}(b)$ for all $a \in A=\left\{z \in I_{k}: x I z\right\}$ and all $b \in B=\left\{z \in I_{k}: x P z\right\}$. Since $y$ belongs to $A$ and $f$ represents $\succsim_{\varphi}$, we have that $f\left(\varphi_{k}(y)\right)>f\left(\varphi_{k+1}(x)\right)$.

- $k=-l<0$. By construction of the ghosts and the extension $\succsim_{\varphi}$ of $\succsim$ for $k=-l<0$, we have $\varphi_{-l+1}(c) \succ_{\varphi} \varphi_{-l}(y) \succsim_{\varphi} \varphi_{-l+1}(d)$ for all $c \in C=\{z \in$ $\left.I_{-l+1}: z P y\right\}$ and all $d \in D=\left\{z \in I_{-l+1}: z I y\right\}$. Since $x$ belongs to $D$ and $f$ represents $\succsim_{\varphi}$, we have that $f\left(\varphi_{-l}(y)\right)=f\left(\varphi_{k}(y)\right) \geq f\left(\varphi_{-l+1}(x)\right)=$ $f\left(\varphi_{k+1}(x)\right)$.

\section{Remark 20}

Choosing a representation $f$ ranging in the ]0,1[ interval is restrictive, as observed in Bouyssou and Pirlot (2020a), in the subsection of Section 4.2 devoted to the construction of a representation. In contrast with the latter paper, we do not intend here to guarantee that all possible numerical representations of the semiorder can be obtained by our construction. Such an opportunity was already lost in the procedure we followed for inserting the ghosts, which is described in Sections 4.4, 4.5 and 4.6. We indeed selected a particular way of inserting the new ghosts in the previously constructed extension of $I_{0}$. Therefore, in the sequel we just aim at showing the existence of a representation.

The next result gives necessary and sufficient conditions for an $I$-connected semiorder to have a strict unit representation. It is thus a generalization of Manders (1981, Prop. 8, p. 237) and Bouyssou and Pirlot (2020a, Prop. 36), which deal with denumerable semiorders. Note that the Bounded $P$-chain condition is implied by I-connectedness (see Bouyssou and Pirlot, 2020a, Prop. 54). Hence it need not be imposed here.

\section{Proposition 21}

Let $S=(P, I)$ be an I-connected semiorder on the set $X$ and let $\succsim$ be its trace. $S$ admits a unit strict representation $u$ satisfying (1) iff the complete preorder $\succsim$ is $d$-separable and $S$ has an at most denumerable set of noses.

\section{ProOF}

Both conditions are necessary. If $u$ is a numerical representation of $S$ and $x \succ y$ then $u(x)>u(y)$. We may impose that $u$ assigns the same value to all the elements in the same equivalence class of $\sim$. In such a case $u$ is a numerical representation of the complete preorder $\succsim$. It is well-known that the existence of a denumerable set $d$-separating $\succsim$ is a necessary and sufficient condition for the existence of a numerical representation of this complete preorder (Krantz et al., 
1971, Th. 2, p. 40). We showed in Remark 11 that no representation satisfying (1) exists when the set of noses is uncountable.

The construction of $\tilde{I}_{0}$ endowed with the complete preorder $\succsim_{\varphi}$ and Proposition 19 establish the existence of a numerical representation of $S$ satisfying (1).

\subsection{Assembling representations on $I$-connected components}

We now consider the general case in which the semiorder $S=(P, I)$ on $X$ is not necessarily $I$-connected. The strategy followed in the denumerable case can also be used in the general case (Bouyssou and Pirlot, 2020a, Remark 64). Assuming that a strict unit representation exists for the restrictions of the semiorder to each of its $I$-connected components, we assemble them into a strict unit representation of the whole semiorder. In order to do so, we need assuming that the semiorder satisfies the Bounded $P$-chain condition. The next proposition is our main result concerning strict unit representations. It is a reformulation of Candeal and Induráin (2010, Th. 3.6, p. 487) in which the $s$-separability condition is factorized into $d$-separability of the trace and denumerability of the set of noses. We emphasize that this factorization refines both the $s$-separability condition and the $P$-gap-edge-points condition of Beja and Gilboa (1992) (see Remark 12 above).

\section{Theorem 22}

Let $S=(P, I)$ be a semiorder on the set $X$ and let $\succsim$ be its trace. $S$ admits a unit numerical representation $u$ satisfying (1) iff the semiorder $S$ satisfies the Bounded $P$-chain condition, the complete preorder $\succsim i s ~ d-s e p a r a b l e ~ a n d ~ S$ has an at most denumerable set of noses. The latter three conditions are independent.

\section{ProOF}

The necessity of the Bounded P-chain condition (Property 1 ) is obvious. If there were an infinite $P$-chain in a interval $[a, b]$ (i.e., the set $\{x: a \precsim x \precsim b\}$ ), it would not be possible to assign a finite value to both $u(a)$ and $u(b)$. A numerical representation $u$ of the semiorder satisfies $u(a)>u(b)$ whenever $a \succ b$. A representation of the semiorder might not be a representation of the trace $\succsim$ in the sense that it might distinguish some equivalent elements, i.e., one might have that $u(a)>u(b)$ for some $a \sim b$. In such a case one may always transform $u$ in a representation $u^{\prime}$ of the semiorder that gives the same value to all elements of each equivalence class of $\succsim$. As a result $u^{\prime}$ is a numerical representation of $\succsim$, which cannot exist if $\succsim$ is not $d$-separable (Krantz et al., 1971, Th. 2, p. 40). It was shown in Remark 11 that a representable semiorder has an at most denumerable set of noses.

We now show the sufficiency of the conditions. Assuming Property 1, Proposition 23 in Bouyssou and Pirlot (2020a) implies that a representation of a semiorder can be built whenever there is a representation of the restrictions of the semiorder 
to each of its $I$-connected components. If the whole semiorder has an at most denumerable set of nodes, it is also the case of its restrictions to all $I$-connected components. If the trace $\succsim$ is $d$-separable so are its restrictions to all $I$-connected components. Therefore, applying Proposition 21, we know that there exists a numerical representation on each I-connected component. Using Remark 64 in Bouyssou and Pirlot (2020a), we know how to assemble them into a representation of the whole semiorder.

To prove the independence of the three conditions, consider the following three examples.

\section{Example 23}

Let $X=\mathbb{R}^{2}$. Consider the binary relation $S$ such that $S=P \cup I$ with $\left(x_{1}, x_{2}\right) P$ $\left(y_{1}, y_{2}\right)$ if $x_{1}>y_{1}+1$ or $\left[x_{1}=y_{1}+1\right.$ and $\left.x_{2}>y_{2}\right]$, while $I$ is the symmetric complement of $P$ (i.e., $x I y \Leftrightarrow \operatorname{Not}[x P y]$ and $\operatorname{Not}\left[\begin{array}{ll}y & P\end{array}\right]$ ).

It is not difficult to show that $S$ is a semiorder (that is not a complete preorder). It is clear that for all $x, y \in X$, there is an $I$-chain joining them, so that this semiorder is $I$-connected and therefore the Bounded $P$-chain condition holds. The set of noses of $S$ is easily seen to be empty. The trace of $S$ is the lexicographic preorder on $\mathbb{R}^{2}$. Hence, $d$-separability is violated (see Beardon et al., 2002, Bridges and Mehta, 1995).

\section{Example 24}

Let $S=(P, I)$ be the semiorder on $\mathbb{R}$ defined by $x P y \Leftrightarrow x \geq y+1$, while $I$ is the symmetric complement of $P$. For all $x, y \in \mathbb{R}$, there is a $P$-chain joining them, so that the Bounded $P$-chain condition holds. The trace $\succsim$ of $S$ is $\geq$ on $\mathbb{R}$, so that the trace is $d$-separable. All ordered pairs $(x, y) \in \mathbb{R}^{2}$ such that $x=y+1$ are noses, which violates the denumerable noses condition.

\section{Example 25}

Let $X=\mathbb{N} \cup\{\omega\}$. Consider the binary relation $S$ such that $\omega P x$, for all $x \in \mathbb{N}$ and $x P y$ iff $x>y+1$, for all $x, y \in \mathbb{N}$, while $I$ is the symmetric complement of $P$. Since $X$ is denumerable, $d$-separability and the condition on noses trivially hold. The Bounded P-chain condition is violated.

\subsection{Hollows-faithful representations}

In this section (which the reader may wish to skip without prejudice for the understanding of the rest of the paper) we investigate the existence of a special type of strict representation that we shall call hollows-faithful representations. The latter is a strict unit representation in which the values associated to the endpoints of 
each hollow differ by exactly one unit. In the construction of the set $\tilde{I}_{0}$ and the complete preorder $\succsim_{\varphi}$ detailed in Sections 4.3 to 4.6, we placed, in some particular cases, the ghost of an element in $I_{k}, k>1$ (resp. in $I_{-l}, l>1$ ) into the equivalence class (w.r.t. $\succsim_{\varphi}$ ) of the ghost of an element in $I_{k-1}$ (resp. in $I_{-l+1}$ ). In the construction of $I_{0,1}$ (see Section 4.3), the ghost $\varphi_{1}(x)$ of an element $x$ in $I_{1}$ is set equivalent to an element $a \in I_{0}$ if and only if $(x, a)$ is a hollow (see also footnote 6 , page 13). When applying (6) to a numerical representation of the complete preorder $\succsim_{\varphi}$ on $\tilde{I}_{0}$, we obtain a strict representation $u$, with $u(x)=u(a)+1$ for all hollows $(x, a), x \in I_{1}, a \in I_{0}$.

However, in all the other steps of the construction of $\left(\tilde{I}_{0}, \succsim_{\varphi}\right)$, there is no guarantee that ghosts put in the same equivalence class always correspond to hollows. This is emphasized in footnotes 8,9 and 10 .

We shall prove below that a semiorder admits a hollows-faithful representation as soon as the semiorder has a strict unit representation. The proof will imply to slightly modify the ghost insertion procedure (Sections 4.4 to 4.6). It also requires to bring some change in the partitions $\left(I_{m}, m \in M\right)$ to avoid that both endpoints of a hollow might belong to the same class $I_{m}$.

\section{Proposition 26 (Existence of hollows-faithful representations)}

Let $S=(P, I)$ be a semiorder on the set $X$ and let $\succsim$ be its trace. If the semiorder $S$ satisfies the Bounded $P$-chain condition, the complete preorder $\succsim$ is d-separable and $S$ has an at most denumerable set of noses, then $S$ admits a unit strict numerical representation u satisfying:

$$
u(x)-u(y)=1 \quad \text { iff } \quad(x, y) \text { is a hollow. }
$$

\section{ProOF}

For proving the result, it suffices to establish the existence of a special strict unit representation satisfying (7) for each $I$-connected component $\mathcal{D}$ of $(X, S)$. Indeed, assembling representations that fulfill (7) as described in Section 4.8 preserves this property. We thus consider any $I$-connected component $\mathcal{D}$ of the semiorder. There are two problems with the current construction of a strict unit representation that need to be solved in view of constructing a representation satisfying (7): (i) both hollow endpoints belonging to consecutive classes of a partition $\left(I_{m}, m \in M\right)$ should be assigned to the same equivalence class of the complete preorder $\succsim_{\varphi}$; (ii) hollow endpoints should not belong to the same class of the partition. We first show how to solve (i) by slightly modifying the ghost insertion procedure described in Sections 4.3 to 4.6. Then we show how to modify a partition $\left(I_{m}, m \in M\right)$ in order to solve (ii) by avoiding the presence of both endpoints of a hollow in the same class.

(i) Hollows-faithful insertion procedure. 
$I_{0,1}:$ no modification.

$I_{0, k}$ : only the insertion of the ghosts of elements $x \in I_{k}$ such that $(x, y)$ is a hollow for some $y \in I_{k-1}$. In such a case, $x$ is the largest element in $J(x)$ and $\varphi_{k-1}\left(A_{x}^{k-1}\right)$ has a least element $\varphi_{k-1}(y)$. Instead of inserting $\varphi_{k}(x)$ between $A_{x}$ and $B_{x}$ as described in Section 4.4, we set $\varphi_{k}(x) \sim_{0, k} \varphi_{k-1}(y)$. This means that we insert $\varphi_{k}(x)$ in between $A_{x} \backslash L(x)$ and $B_{x} \cup L(x)$, setting it indifferent to a least element in $A(x) \backslash L(x)$. With this modified insertion, Lemma 15 remains true. Its proof need only be adapted in Cases 3 and 4 . Regarding Case 3, nothing changes unless $x_{1}$ is the endpoint of a hollow $\left(x_{1}, y\right)$ with $y \in I_{k-1}$. In such a case, we have $\varphi_{k}\left(x_{1}\right) \sim_{0, k} \varphi_{k-1}(y)$. If $z_{1}=\varphi_{k}\left(x_{1}\right)$ and $z_{2} \in I_{0, k-1}$, we have that $z_{1} \succ_{0, k} z_{2}$ implies that $z_{2} \in B_{x_{1}} \cup L\left(x_{1}\right)$. By the induction hypothesis, there is $z \in E_{0, k-1}$ which separates $\varphi_{k-1}(y)$ and $z_{2}$, i.e., $\varphi_{k-1}(y) \succsim_{0, k-1} z \succsim_{0, k-1} z_{2}$. Since $z_{1} \sim_{0, k} \varphi_{k-1}(y)$ and $\succsim_{0, k}$ extends $\succsim_{0, k-1}$, we have that $z \in E_{0, k-1}$ also separates $z_{1}$ and $z_{2}$ in $\left(I_{0, k}, \succsim_{0, k}\right)$. A similar argument applies in Case 4.

$I_{-1, k}$ : only the insertion of the ghosts of elements $x \in I_{-1}$ such that $(y, x)$ is a hollow for some $y \in I_{0}$. In such a case, $x$ is the least element in $J(x)$ and $\varphi_{-1}\left(D_{x}^{-1}\right)$ has a largest element $y$. Instead of inserting $\varphi_{-1}(x)$ between $C_{x}$ and $D_{x}$ as described in Section 4.5, we set $\varphi_{-1}(x) \sim_{-1, k} y$. This means that we insert $\varphi_{-1}(x)$ in between $D_{x} \backslash M(x)$ and $C_{x} \cup M(x)$, setting it indifferent to a largest element in $D(x) \backslash M(x)$. With this modified insertion, Lemma 16 remains true. Its proof need only be adapted in Cases 3 and 4. Regarding Case 3, nothing changes unless $x_{1}$ is the endpoint of a hollow $\left(y, x_{1}\right)$ with $y \in I_{0}$. In such a case, we have $\varphi_{-1}\left(x_{1}\right) \sim_{-1, k} y$. If $z_{1}=\varphi_{-1}\left(x_{1}\right)$ and $z_{2} \in I_{0, k}$, we have that $z_{1} \succ_{-1, k} z_{2}$ implies that $z_{2} \in D_{x_{1}} \cup M\left(x_{1}\right)$. By the induction hypothesis, there is $z \in E_{0, k}$ which separates $y$ and $z_{2}$, i.e., $y \succsim_{0, k} z \succsim_{0, k} z_{2}$. Since $z_{1} \sim_{-1, k} y$ and $\succsim_{-1, k}$ extends $\succsim_{0, k}$, we have that $z \in E_{0, k}$ also separates $z_{1}$ and $z_{2}$ in $\left(I_{-1, k}, \succsim_{-1, k}\right)$. A similar argument applies in Case 4.

$I_{-l, k}$ : only the insertion of the ghosts of elements $x \in I_{-l}$ such that $(y, x)$ is a hollow for some $y \in I_{-l+1}$. In such a case, $x$ is the least element in $J(x)$ and $\varphi_{-1}\left(D_{x}^{-l+1}\right)$ has a largest element $\varphi_{-l+1}(y)$. Instead of inserting $\varphi_{-l}(x)$ between $C_{x}$ and $D_{x}$ as described in Section 4.6, we set $\varphi_{-l}(x) \sim_{-l, k} \varphi_{-l+1}(y)$. This means that we insert $\varphi_{-l}(x)$ in between $D_{x} \backslash M(x)$ and $C_{x} \cup M(x)$, setting it indifferent to a largest element in $D(x) \backslash M(x)$. With this modified insertion, Lemma 17 remains true. Its proof need only be adapted in Cases 3 and 4. Regarding Case 3, nothing changes unless $x_{1}$ is the endpoint of a hollow $\left(y, x_{1}\right)$ with $y \in I_{-l+1}$. In such a case, we have $\varphi_{-l}\left(x_{1}\right) \sim_{-l, k} \varphi_{-l+1} y$. If $z_{1}=\varphi_{-l}\left(x_{1}\right)$ and $z_{2} \in I_{-l+1, k}$, we have that $z_{1} \succ_{-l, k} z_{2}$ implies that $z_{2} \in D_{x_{1}} \cup M\left(x_{1}\right)$. By the induction hypothesis, there is $z \in E_{-l+1, k}$ which 
separates $\varphi_{-l+1}(y)$ and $z_{2}$, i.e., $\varphi_{-l+1}(y) \succsim_{-l+1, k} z \succsim_{-l+1, k} z_{2}$. Since $z_{1} \sim_{-l, k} y$ and $\succsim_{-l, k}$ extends $\succsim_{-l+1, k}$, we have that $z \in E_{-l+1, k}$ also separates $z_{1}$ and $z_{2}$ in $\left(I_{-l, k}, \succsim_{-l, k}\right)$. A similar argument applies in Case 4.

Applying the usual construction of a representation described in Section 4.7, we obtain a strict unit representation of the semiorder restricted to the $I$-connected component $\mathcal{D}$. Such a representation fulfills $(7)$ for all hollows $(x, y)$ for which $x$ and $y$ belong to different indifference classes $I_{m}$ and $I_{m+1}$.

(ii) Modifying the partition $\left(I_{m}, m \in M\right)$.

With the construction of the partition $\left(I_{m}, m \in M\right)$ described in Bouyssou and Pirlot (2020a, Section 3), it may happen that both endpoints of a hollow belong to the same indifference class $I_{m}$ (see Example 27 below). In order to obtain a partition $\left(I_{m}^{\prime}, m \in M^{\prime}\right)$ without hollows inside a class $I_{m}^{\prime}$, for all $m \in M^{\prime}$, we may restructure the initial partition as follows.

- Search for the smallest $k \geq 0$ such that $I_{k}$ contains both endpoints $x, y$ of a hollow $(x, y)$. If this is the case for $I_{k}$, let $I_{k}^{\prime}=I_{k} \backslash\{x\}$. Transfer $x$ to $I_{k+1}^{\prime}$ and, define $I_{k+1}^{\prime}$ as the maximal indifference class whose least element is $x$; however, if this class has a greatest element $z$, and $(z, x)$ is a hollow, then transfer $z$ to $I_{k+2}$. Continue in this way, adapting the construction principles explained in Bouyssou and Pirlot (2020a, Section 3) in order to avoid the existence of hollows with both endpoints in the same class. If no $I_{k}, k \geq 0$ containing both endpoints of a hollow is found, keep the classes $\left(I_{k}, k \geq 0\right)$ unchanged.

- Search for the smallest $l>0$ such that $I_{-l}$ contains both endpoints $x, y$ of a hollow $(x, y)$. If this is the case for $I_{-l}$, let $I_{-l}^{\prime}=I_{-l} \backslash\{y\}$. Transfer $y$ to $I_{-l-1}^{\prime}$ and, define $I_{-l-1}^{\prime}$ as the maximal indifference class whose largest element is $y$; however, if this class has a least element $w$, and $(y, w)$ is a hollow, then transfer $w$ to $I_{-l-2}$. Continue in this way, adapting the construction principles explained in Bouyssou and Pirlot (2020a, Section 3) in order to avoid the existence of hollows with both endpoints in the same class. If no $I_{-l}, l>0$ containing both endpoints of a hollow is found, keep the classes $\left(I_{-l}, l \geq 0\right)$ unchanged.

The obtained partition $\left(I_{m}^{\prime}, m \in M^{\prime}\right)$ has no class which contains both endpoints of a hollow. Applying the modified ghost insertion procedure described in the beginning of this proof to the partition $\left(I_{m}^{\prime}, m \in M^{\prime}\right)$ yields a strict unit representation that fulfills condition (7).

We illustrate the previous result and its proof in the following example. 


\section{Example 27}

Consider the semiorder $S=(P, I)$ defined on a set $X$ as follows. Let $X$ be the following union of real intervals: $X=[-0.5,1.9[\cup[2.1,2.9] \cup] 3.1,5.1]$ endowed with the restriction to $X$ of the usual strict unit semiorder on $\mathbb{R}$. Assume that the partition $I_{m}, m=-1,0, \ldots 5$ is the one represented in Figure 5 .

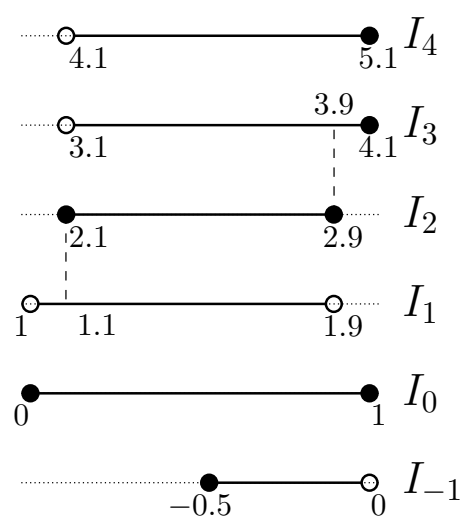

Figure 5: Partition in indifference classes for the semiorder $(X, S)$. An empty (resp. filled) circle indicates that the segment is open (resp. closed).

The pair $(2.9,2.1)$ in $I_{2}$ is a hollow. In the numerical representation that defines the semiorder, the difference between the values associated to the endpoints of this hollow is equal to 0.8. The question we want to solve is whether there is a strict unit representation of this semiorder in which the endpoints values of all hollows differ by exactly one unit. Below, we modify the partition $\left(I_{m}, m=-1, \ldots, 4\right)$ in order to avoid having hollow endpoints in the same class (see Figure 6). Then we illustrate the application of the modified ghost insertion procedure described in the proof of Proposition 26.

Let us start with $I_{0}$. This class contains the hollow $(1,0)$. We move 1 to $I_{1}^{\prime}$. $I_{1}^{\prime}=\left[1,1.9\left[\right.\right.$. In $I_{2},(2.9,2.1)$ is a hollow. We move 2.9 to $I_{3}^{\prime}$, leaving $I_{2}^{\prime}=[2.1,2.9[$. $I_{3}^{\prime}$ starts with 2.9 up to 3.9. We do not include 3.9 in $I_{3}^{\prime}$ in order to avoid including the hollow $(3.9,2.9)$ in a class. So $I_{3}^{\prime}=\{2.9\} \cup\left[3.1,3.9\left[. I_{4}^{\prime}=[3.9,4.9[\right.\right.$. Finally, $I_{5}^{\prime}=[4.9,5.1]$. There is one more class than in the original partition.

The modified ghost insertion procedure, applied to $\left(I_{m}^{\prime}, m=-1, \ldots, 5\right)$, yields $\tilde{I}_{0}=I_{-1,5}$ ordered by $\succsim_{\varphi}$, as represented in Table 1 . We start with the insertion of the ghosts of the elements of $I_{1}^{\prime}$ into $I_{0}^{\prime}$. All elements of $I_{1}^{\prime}$ are the upper endpoint of a hollow, the other endpoint being in $1_{0}^{\prime}$. Therefore, for all $x \in I_{1}^{\prime}=[1,1.9[$, its ghost $\varphi_{1}(x)$ is equivalent to the other endpoint $y$ of the hollow $(x, y)$, i.e., $\varphi_{1}(x) \sim_{0,1} y=x-1$. This is shown in the rows labeled $I_{0}^{\prime}$ and $I_{1}^{\prime}$ in Table 1. Important elements of $I_{0}^{\prime}$ have been singled out, namely, $0,0.1,0.5$ and 0.9 , because 


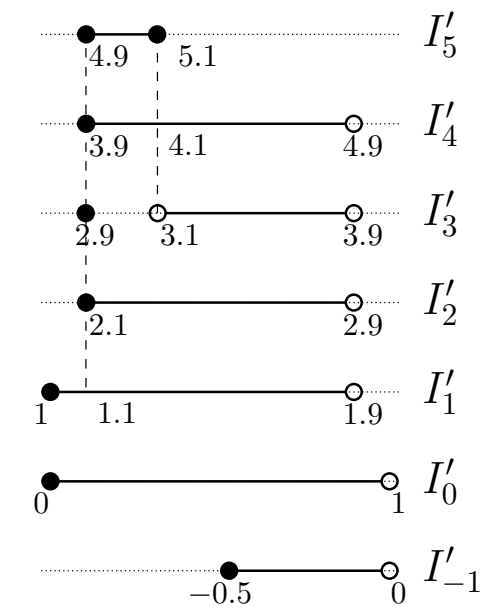

Figure 6: Modified partition $\left(I_{m}^{\prime}, m=-1, \ldots, 5\right)$ for the semiorder $(X, S)$

they correspond to transitions in $\tilde{I}_{0}(0.3$ does not correspond to a particular element in $I_{0}^{\prime}$; it is used to create a gap in which some ghosts from $I_{4}^{\prime}$ and $I_{5}^{\prime}$ need to be inserted). We see that the endpoints of each hollow are inserted in the same column of the table (single hollow endpoints in the columns headed by singular values; intervals coupled in the hollow relation in intervals between singular values). Each element in $I_{2}^{\prime}=\left[2.1,2.9\left[\right.\right.$ is associated to an element of $\left[1.1,1.9\left[\subset I_{1}^{\prime}\right.\right.$ by the hollow relation. Therefore, we have, for all $x \in I_{2}^{\prime}, \varphi_{2}(x) \sim_{0,2} \varphi_{1}(x-1) \sim_{0,2} x-2$. The row labelled $I_{2}^{\prime}$ in Table 1 shows that the elements in $I_{2}^{\prime}$ are assigned to the same columns as their associate elements through the hollow relation. The insertion of the ghosts of $I_{3}^{\prime}$ is similarly constrained by the hollow relation. The least element 2.9 is associated to 2.1 because they form a hollow. Therefore the ghosts of $2.9,2.1,1.1$ and 0.1 are indifferent w.r.t. $\sim_{0,3}$. The elements of ]3.1,3.9[ are coupled to those of ]2.1,2.9[. This is shown in Table 1 where the elements of ] $3.1,3.9$ [ are assigned to the columns between 0.3 and 0.9 just as the corresponding elements of $] 2.1,2.9\left[\right.$. It is the insertion of $I_{4}^{\prime}$ that creates the gap in the table. The least element 3.9 is coupled to 2.9 and placed in the column headed by 0.1 . The elements in $] 3.9,4.1$ ] are not upper endpoints of a hollow. Their ghosts have to be inserted between those of 2.9 and those of all the elements of the interval ]3.1,3.9[. Therefore, they are positioned in the table in the column between 0.1 and 0.3 and 4.1 is assigned to the column headed (arbitrarily) by 0.3 . The elements $I_{5}^{\prime}$ are coupled to these in $[3.9,4.1] \subset I_{4}^{\prime}$. Their position in the table results from this constraint. Finally, since the elements of $I_{-1}^{\prime}=[-0.5,0[$ are coupled to the elements in $\left[0.5,1\left[\subset I_{0}^{\prime}\right.\right.$ by the hollow relation, forcing the position indicated in the table. 
We are now in position to explain the headings row in the table. The row label $f$ refers to a representation of the complete preorder $\succsim_{-1,5}=\succsim_{\varphi}$ on $\tilde{I}_{0}$. If we assign the heading value to the ghosts of the elements in the columns having a heading and if we linearly interpolate between these values to assign values to the ghosts in the intervals between the headed columns, we obtain a representation of $\succsim_{\varphi}$. From this, we build a representation of the semiorder, which fulfills condition (7), by using definition (6).

\begin{tabular}{|l|l|l|l|l|l|l|l|l|l|l|l|}
\hline$f$ & 0 & & 0.1 & & 0.3 & & 0.5 & & 0.9 & & 1 \\
\hline$I_{0}^{\prime}$ & 0 & ] $0,0.1[$ & 0.1 & & & ] $0.1,0.5[$ & 0.5 & ] $0.5,0.9[$ & 0.9 & ] $0.9,1[$ & \\
$I_{1}^{\prime}$ & 1 & ] $1,1.1[$ & 1.1 & & & ] $1.1,1.5[$ & 1.5 & ] $1.5,1.9[$ & & & \\
$I_{2}^{\prime}$ & & & 2.1 & & & ] $2.1,2.5[$ & 2.5 & ] $2.5,2.9[$ & & & \\
$I_{3}^{\prime}$ & & & 2.9 & & & ] $3.1,3.5[$ & 3.5 & ] $3.5,3.9[$ & & & \\
$I_{4}^{\prime}$ & & & 3.9 & ] $3.9,4.1[$ & 4.1 & ] $4.1,4.5[$ & 4.5 & ] $4.5,4.9[$ & & & \\
$I_{5}^{\prime}$ & & & 4.9 & ] $4.9,5.1[$ & 5.1 & & & & & & \\
$I_{-1}^{\prime}$ & & & & & & & -.5 & ]$-.5,-.1[$ & -.1 & ]$-.1,0[$ & \\
\hline
\end{tabular}

Table 1: $\tilde{I}_{0}$ and a function $f$ that represents the complete preorder $\succsim_{\varphi}$ on $\tilde{I}_{0}$

Figure 7 represents in an obvious manner the semiorder as well as the hollowsfaithful strict unit representation obtained by applying (6) to the numerical representation $f$ of the complete preorder $\succsim_{\varphi}$ on $\tilde{I}_{0}$.

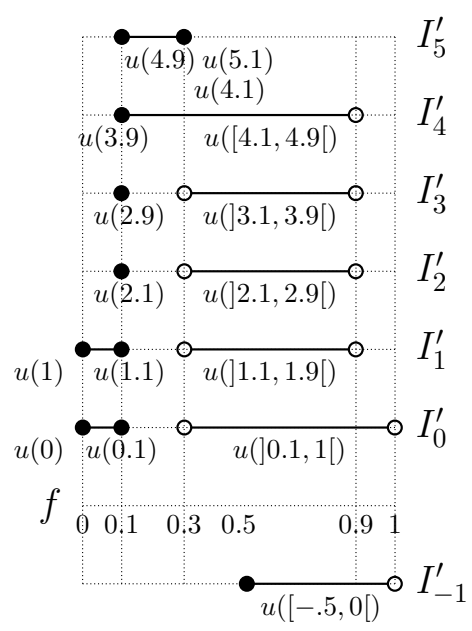

Figure 7: Hollows-faithful strict unit representation of the semiorder $(X, S)$

\section{Remark 28}

The result in Proposition 26, i.e., the existence of hollows-faithful representations as soon as strict unit representations exist, is not limited to uncountable 
semiorders. Of course it holds - and so does the proof - for denumerable semiorders under the Bounded P-chain condition, which guarantees the existence of a strict unit representation (Bouyssou and Pirlot, 2020a, Th. 64). We did not mention this result in Bouyssou and Pirlot (2020a) because our goal there was mainly to study the "uniqueness" of the representation. Therefore we did not focus on special representations but tried to show how to construct all possible representations.

\subsection{Summary of results}

In Section 4:

- We gave interpretable conditions for the existence of a strict unit representation of a semiorder in the general case. Our denumerable set of noses condition refines both the $s$-separability condition of Candeal and Induráin (2010) and the P-gap-edge-points of Beja and Gilboa (1992). The relationship between the latter two conditions is thus clarified.

- We showed that the technique for constructing a strict unit representation, that we developed in Bouyssou and Pirlot (2020a) in the denumerable case, can be adapted to deal with the general case. In addition, we showed that such a construction gives control on the representation. This assertion is illustrated by establishing the existence of special representations, namely the hollows-faithful representations.

The benefit of giving a particular role to noses and hollows will be further emphasized in the next section, which studies the existence of nonstrict unit representations. We shall see that, for nonstrict unit representations, the hollows play the role played by the noses in the case of strict unit representations.

\section{$5 \quad$ Nonstrict unit representations}

Conditions guaranteeing that a semiorder has a nonstrict unit representation, i.e., a representation satisfying (2), have been established by Beja and Gilboa (1992, Th. 4.5.b, p. 439). Candeal and Induráin (2010) did not study nonstrict unit representations but a condition "dual" to their $s$-separability condition can be formulated. The latter will play the role of $s$-separability for nonstrict unit representations. We shall write down such a condition and factorize it into $d$-separability of the trace and another condition, in the spirit of what we did for strict unit representations. The latter condition refines both the condition dual to $s$-separability and the condition of denumerability of the set of $I$-upper-edge-points used by Beja and Gilboa (1992) in their characterization. 
In the case $X$ is finite, all semiorders have both strict and nonstrict representations (see, e.g., Pirlot and Vincke (1997), Section 4.2). In case $X$ is countably infinite, every semiorder that admits a strict unit representation has also a nonstrict unit representation, and conversely (Beja and Gilboa (1992, Th. 3.8); this has already been observed by Roberts (1979, footnote, p. 36)). It is even true (Bouyssou and Pirlot, 2020a, Th. 64) that a semiorder on a denumerable set that admits a unit representation also has a unit representation $u$ that is simultaneously strict and nonstrict (i.e., $|u(x)-u(y)| \neq 1$, for all $x, y$ ). The Bounded $P$-chain condition is a necessary and sufficient condition for a semiorder on a denumerable set to have both strict and nonstrict unit representations. For a semiorder on an uncountable set it is no longer the case that it has a strict unit representation if and only if it has a nonstrict one. Consider for instance the nonstrict unit semiorder on the reals (Example 24). It has a trivial nonstrict unit representation. The set of its noses is not denumerable. Therefore, by Theorem 22, this semiorder has no strict unit representation. So, the two types of representation are not equivalent when $X$ is not denumerable.

Our goal in this section is to establish a new characterization of the semiorders that admit a nonstrict unit representation.

\subsection{Separability for nonstrict unit representations}

We introduce another form of separability that can be viewed as "dual" w.r.t. $s$ separability (Candeal and Induráin, 2010) and is useful to characterize semiorders that admit a nonstrict unit representation.

\section{Definition 29 ( $s^{\prime}$-separability)}

A semiorder $S=(P, I)$ is $s^{\prime}$-separable if there is a denumerable set $F, F \subseteq X$, such that, for all $a, b \in X$ with $a S b$, there are

$$
\begin{array}{rll}
c \in F & \text { such that } & a S c \succsim b \\
\text { and } d \in F & \text { such that } & a \succsim d S b .
\end{array}
$$

This notion of separability implies that the number of hollows (see Definition 5) of the semiorder is at most denumerable. The following lemma is the counterpart of Lemma 7 in the context of $s^{\prime}$-separability.

\section{Lemma 30}

If the semiorder $S=(P, I)$ on $X$ is $s^{\prime}$-separable by the denumerable set $F$, then a and $b$ belong to $F$ whenever $(a, b)$ is a hollow.

\section{PROOF}

Let $(a, b)$ be a hollow, thus satisfying $b I a$ hence $b S a$. By the $s^{\prime}$-separability property, there is $c \in F$ such that $b S c \succsim a$. By definition of a hollow, for all $c \succ a$, we 
have $c P b$. Therefore, $c=a$ and $a \in F$. Using $s^{\prime}$-separability, there is also $d \in F$ such that $b \succsim d S a$, which implies $b=d$ and $b \in F$.

Before proving a factorization result similar to Proposition 10, we introduce the notion of half-hollow, adapting the notion of half-noses introduced in Definition 8.

\section{Definition 31 (Half-hollows)}

The ordered pair $(a, b) \in X \times X$ is a lower half-hollow (l-h-hollow) of $S=(P, I)$ if $a I b, a \succ b$, and there is no $d \in X$ such that $b \succ d I a .(a, b)$ is a proper l-h-hollow if it is a l-h-hollow and there is $c \in X$ such that $b I c \succ a .(a, b) \in X \times X$ is an upper half-hollow (u-h-hollow) of $S=(P, I)$ if $a I b, a \succ b$, and there is no $c \in X$ such that $b I c \succ a$. $(a, b)$ is a proper $u$-h-hollow if it is an $u$-h-hollow and there is $d \in X$ such that $b \succ d I a$.

Let LHH (resp. UHH) denote the set of right endpoints b (resp. left enpoints a) of all proper l-h-hollows (resp. u-h-hollow) $(a, b)$.

The notion of upper half-hollow is closely related to that of I-upper-edge point (Beja and Gilboa, 1992, Definition P6 (b), p.438). The element $a \in X$ is an $I$-upper-edge-point if and only if there is $b \in X$ such that $(a, b)$ is an upper halfhollow. We have the following result. It is the counterpart of Lemma 9 and its proof is similar. We give it for the reader's convenience.

\section{Lemma 32}

If the semiorder $S=(P, I)$ is d-separable, then the sets $L H H$ and $U H H$ are denumerable.

\section{Proof}

We only prove the result for $L H H$. The other case is dealt with similarly.

Let $(a, b)$ be a proper l-h-hollow. We define the set $H(b)=\{x \in X: x I b, x \succ$ $b$ and $\forall d \prec b, N o t[x I d]\}$. In other words, for all $x \in H(b),(x, b)$ is a l-h-hollow. It is clear that $a \in H(b)$. Moreover, since $(a, b)$ is proper, $H(b)$ contains at least one other element $c$ such that $b I c \succ a$. $H(b)$ is an interval w.r.t. $\succ$. To show this let $x, x^{\prime} \in H(b)$. If $x^{\prime \prime}$ is such that $x \succ x^{\prime \prime} \succ x^{\prime}$, then $x^{\prime \prime} \in H(b)$. Indeed, $x^{\prime \prime} I b$ since $x I b$ and for all $d \prec b, \operatorname{Not}\left[x^{\prime \prime} I d\right]$ since $\operatorname{Not}\left[x^{\prime} I c\right]$.

Let $(a, b)$ and $\left(a^{\prime}, b^{\prime}\right)$ be two proper l-h-hollows, with $b \neq b^{\prime} \in L H H$. It is clear that the associated intervals $H(b)$ and $H\left(b^{\prime}\right)$ are disjoint. Each of these intervals contains at least two distinct points and therefore at least an element from the denumerable set $D$ that $d$-separates $S=(P, I)$. Consequently, $L H H$ is denumerable.

\section{Proposition 33}

A semiorder $S=(P, I)$ on $X$ is $s^{\prime}$-separable iff $\succsim$ is $d$-separable and the set of hollows is denumerable. 


\section{PROOF}

Assume that the semiorder is $s^{\prime}$-separable. By Lemma 30, the set of hollows is denumerable. The $s^{\prime}$-separability property implies that $\succsim$ is $d$-separable. Let $x, y \in X$ be such that $x \succ y$. There is $z \in X$ such that $x P z$ and $z S y$ and/or $w \in X$ such that $w P y$ and $x S w$. In the former case, $s^{\prime}$-separability entails that there is $c \in F$ such that $z S c \succsim y$ and, since $x P z$, we have $x \succ c \succsim y$. In the latter case, there is $d \in F$ such that $x \succsim d S w$ and, since $w P y$, we have $x \succsim d \succ y$.

Reciprocally, let $D$ be a denumerable set that $d$-separates $\succsim$. Let $x, y \in X$ be such that $y S x$. We first deal with the case $y \succ x$. By $d$-separability, there is $z \in D$ such that $x \precsim z \precsim y$, which implies $y S z$ and $z S x$. Therefore we have $y S z \succsim x$ and $y \succsim z S x$. The other case is when $x \succ y$. If $(x, y)$ is a hollow, the $s^{\prime}$-separability condition is trivially satisfied. Assuming that $(x, y)$ is not a hollow, we have that

1. either there is $x^{\prime} \succ x$ such that $y S x^{\prime}$

2. or there is $y^{\prime} \prec y$ such that $y^{\prime} S x$.

In Case 1, by the $d$-separability of $\succ$, there is $c \in D$ such that $x^{\prime} \succsim c \succsim x$. Therefore we have $y S c \succsim x$. Further, there are two cases. Either there is $y^{\prime} \prec y$ such that $y^{\prime} S x$ or for all $y^{\prime} \prec y$, we have $\operatorname{Not}\left[y^{\prime} S x\right]$. In the former case, $d$-separability implies that there is $d \in D$ such that $y^{\prime} \precsim d \precsim y$. Then, we have $y \succsim d S x$. Otherwise, $(x, y)$ is a proper l-h-hollow. In order to have $d \in F$ such that $y \succsim d S x$, we set $d=y$ and include the denumerable set $L H H$ of right endpoints of the proper l-h-hollows in $F$.

In Case 2, by the $d$-separability of $\succ$, there is $d \in D$ such that $y^{\prime} \precsim d \precsim y$. Therefore we have $x S c \precsim y$. Further, either there is $x^{\prime} \succ x$ such that $y S x^{\prime}$ or for all $x^{\prime} \succ x$, we have $\operatorname{Not}\left[y S x^{\prime}\right]$. In the former case, $d$-separability implies that there is $c \in D$ such that $x^{\prime} \succsim c \succsim x$. Then, we have $y S c \succsim x$. Otherwise, $(x, y)$ is a proper u-h-hollow. In order to have $c \in F$ such that $y S c \succsim x$, we set $c=x$ and include the denumerable set $U H H$ of left endpoints of the proper u-h-hollows in $F$.

Finally, by considering $F$ as the union of $D, L H H, U H H$ and the set of elements $a, b$ such that $(a, b)$ is a hollow, which is denumerable by hypothesis, we obtain a denumerable set $F$, which $s^{\prime}$-separates the semiorder $(P, I)$.

\section{Remark 34}

It is easy to show that having a denumerable set of hollows is a necessary condition for a semiorder to have a nonstrict unit representation. Indeed, assume that $u$ is a nonstrict unit representation of the semiorder $S=(P, I)$ and $(a, b)$ is a hollow of $S$. Since $b S a$, we have $u(b)>u(a)-1$. Let $\varepsilon_{a b}$ be the positive number $u(b)-u(a)+1$. By definition of a hollow, there is no element $c \in X$ such that $b \succ c S a$ and therefore, there is no $c$ such that $u(c) \in] u(a)-1, u(b)]$, an interval of length 
$\varepsilon_{a b}>0$. To each hollow $(a, b)$ is associated such an interval of positive length and all these intervals are disjoint. Since sets of disjoint intervals of positive length in $\mathbb{R}$ have a finite or denumerable number of elements, the number of hollows is denumerable.

\section{Remark 35}

For proving the existence of a nonstrict unit representation, we shall use $d$-separability and the condition that the number of hollows is denumerable. We could do it under the $s^{\prime}$-separability assumption, but, in the proof, we shall only use the denumerable set $D$ that is dense in the trace $\succsim$ and the denumerable set of hollows endpoints. We do not need to add the half-noses or the half-hollows as we had to do in the second part of the proof of Proposition 33. In other words, we do not use all the points in the set $F$ involved in the $s^{\prime}$-separability property (see Definition 29). In the same vein, we do not need to impose that the set of all I-upper-edge-points (Beja and Gilboa, 1992, p. 438) is denumerable. Only the cardinality of the set of $I$-upper-edge-points that correspond to hollows needs to be controlled. Our condition that the set of hollows has to be denumerable refines both the $s^{\prime}$-separability condition and the denumerability of the set of $I$-upperedge-points (Beja and Gilboa, 1992, Theorem 4.5 (b), p. 439). Its interpretation is straightforward and it clarifies the relationship between $s^{\prime}$-separability and the conditions imposed by Beja and Gilboa (1992).

\subsection{Characterization of the semiorders admitting a non- strict unit representation}

The insertion process leading to prove the existence of a strict unit representation of a semiorder can be slightly modified to accomodate nonstrict unit representations. In nonstrict representations the hollows play the role of the noses in strict representations and vice versa. We shall prove that there is a nonstrict representation of a semiorder if and only if the semiorder satisfies the Bounded $P$-chain condition, its trace is $d$-separable and the semiorder has a denumerable set of hollows.

As in Section 4, we start by proving that these conditions are sufficient. We first assume that the semiorder $S=(P, I)$ on $X$ has been decomposed into $I$-connected components. Let $\mathcal{D}$ be such a connected component. Assuming that the restriction to $\mathcal{D}$ of the semiorder has a denumerable set of hollows and that its trace is $d$ separable, we adapt the construction of $\left(\tilde{I}_{0}, \succsim_{\varphi}\right)$ described in Sections 4.4 to 4.6 in order to prove that the restriction to $\mathcal{D}$ of the semiorder admits a nonstrict unit representation (as in Section 4.7). Let $E=E^{\prime} \cup H$ be the denumerable set that is the union of all hollows endpoints $H$ and a denumerable subset $E^{\prime}$ of $X$ which 
$d$-separates the trace $\succsim$. Using the notation defined in Section 4.2 , we describe the changes in the construction of $I_{-l, k}$, for all $-l, k \in M$ with $l>0, k \geq 0$, below.

Construction of $I_{0,1}$ Ghost insertion goes as described in Section 4.3. The only difference relates to the positioning of the ghosts of the noses left endpoints (instead of the hollows left endpoints). Whenever $\left(x_{1}, b\right)$ is a nose, with $x_{1} \in I_{1}$ and $b \in I_{0}$, we place the ghost $\varphi_{1}\left(x_{1}\right)$ of $x_{1}$ in the equivalence class of $b$ in the relation $\succsim_{0,1}$. Note that, for a hollow $\left(x_{1}, a\right)$, the ghost $\varphi_{1}\left(x_{1}\right)$ is positioned strictly in between the classes of the bipartition $\left(A_{x_{1}}^{0}, B_{x_{1}}^{0}\right)$, i.e., in such a way that we have $a \succ_{0,1} \varphi_{1}\left(x_{1}\right) \succ_{0,1} b$, for all $b \in B_{x_{1}}^{0}$. There is no change in the proof that the relation $\succsim_{0,1}$ that is obtained is a complete preorder on $I_{0,1}$ extending the trace $\succsim$ on $I_{0}$ and reproducing on $\varphi_{1}\left(I_{1}\right)$ the trace order on $I_{1}$. Slight adaptation is needed to show that this complete preorder is $d$-separated by $E_{0,1}$. These occur in Cases 3 and 4 in the proof of Lemma 14. Let $z_{1} \succ_{0,1} z_{2}$ with $z_{1}, z_{2} \in I_{0,1}$.

Case 3 We consider the case $z_{1}=\varphi_{1}\left(x_{1}\right)$ and $z_{2} \in I_{0}$. We have that $z_{1} \succ_{0,1}$ $z_{2}$ implies $z_{2} \in B_{x_{1}}^{0}$. The only subcase that differs from Lemma 14 is when $x_{1}, z_{2}$ is a nose, i.e., when $z_{2}$ is the greatest element in $B_{x_{1}}^{0}$ and $x_{1}$ is the least element in $J\left(x_{1}\right)$. Actually, such a case does not occur since we have chosen to put $z_{1}=\varphi_{1}\left(x_{1}\right)$ in the equivalence class of $z_{2}$, i.e., to set $\varphi_{1}\left(x_{1}\right) \sim_{0,1} z_{2}$.

Case 4 This case deals with $z_{1} \in I_{0}$ and $z_{2}=\varphi_{1}\left(x_{2}\right)$. We have that $z_{1} \succ_{0,1}$ $z_{2}$ implies that $z_{1} \in A_{x_{2}}^{0}$. The only subcase that differs from Lemma 14 is when $z_{1}$ is the least element in $A_{x_{2}}^{0}$ and $x_{2}$ is the greatest element such that $z_{1} \succsim_{0,1} \varphi_{1}\left(x_{2}\right)$. This means that $\left(x_{2}, z_{1}\right)$ is a hollow. Since $x_{2}$ is a hollow endpoint, it belongs to $E_{0}, z_{2}=\varphi_{1}\left(x_{2}\right)$ belongs to $E_{0,1}$ and it separates $z_{1}$ from $z_{2}$.

This establishes Lemma 14 in the construction process of a nonstrict unit representation.

Construction of $I_{0, k}$ Ghosts are inserted as in Section 4.4. The bipartition $\left(A_{x}, B_{x}\right)$ of $I_{0, k-1}$ associated to element $x$ in $I_{k}$ is defined slightly differently. We choose to include in $B_{x}$ (instead of $A_{x}$ ) the set $L(x)$ of elements of $I_{0, k-2}$ which lie between $\varphi_{k-1}\left(A_{x}^{k-1}\right)$ and $\varphi_{k-1}\left(B_{x}^{k-1}\right)$. We have accordingly:

$A_{x}=\left\{y \in I_{0, k-1}: \exists a \in \varphi_{k-1}\left(A_{x}^{k-1}\right)\right.$ such that $\left.y \succsim_{0, k-1} a\right\}$,

$B_{x}=I_{0, k-1} \backslash A_{x}=L(x) \cup\left\{z \in I_{0, k-1}: \exists b \in \varphi_{k-1}\left(B_{x}^{k-1}\right)\right.$ such that $\left.b \succsim_{0, k-1} z\right\}$.

The definition of $\succsim_{0, k}$ is the same as in Section 4.4 except for Case 3, i.e., when $z_{1}=\varphi_{k}\left(x_{1}\right)$ and $z_{2} \in I_{0, k-1}$. Like in Section 4.4, we have $z_{1} \succ_{0, k} z_{2}$ 
iff $z_{2} \in A_{x_{1}}$ and $z_{2} \succsim_{0, k} z_{1}$ iff $z_{2} \in B_{x_{1}}$. The only difference is the following. When $z_{2}$ is a greatest element in $B_{x_{1}}$ (w.r.t. $\succsim_{0, k-1}$ ) and $x_{1}$ is the least element in $J\left(x_{1}\right)$ (w.r.t. $\succsim$ ), we set $z_{2} \sim_{0, k} z_{1}$.

The proof of Lemma 15 requires adaptation only in Cases 3 and 4 . Let $z_{1}, z_{2} \in I_{0, k}$ be such that $z_{1} \succ_{0, k} z_{2}$.

Case 3 If $z_{1}=\varphi_{k}\left(x_{1}\right)$ and $z_{2} \in I_{0, k-1}$, we have that $z_{1} \succ_{0, k} z_{2}$ implies that $z_{2} \in B_{x_{1}}$. The only subcase that differs from Lemma 15 is when $z_{2}$ is the greatest element in $B_{x_{1}}$ and $x_{1}$ is the least element in $J\left(x_{1}\right)$. Actually, in such a case, we chose to put $z_{1}=\varphi_{1}\left(x_{1}\right)$ in the equivalence class of $z_{2}$, i.e., to set $\varphi_{1}\left(x_{1}\right) \sim_{0,1} z_{2}$, which is not compatible with $z_{1} \succ_{0, k} z_{2}$.

Case 4 If $z_{1} \in I_{0, k-1}$ and $z_{2}=\varphi_{k}\left(x_{2}\right)$, we have that $z_{1} \succ_{0, k} z_{2}$ implies that $z_{1} \in A_{x_{2}}$. The only subcase that differs from Lemma 15 is when $z_{1}$ is a least element in $A_{x_{2}}$ and $x_{2}$ is the greatest element in $J\left(x_{2}\right)$. such that $z_{1} \succsim_{0,1} \varphi_{1}\left(x_{2}\right)$. This means that $\left(x_{2}, z_{1}\right)$ is a hollow. Since $x_{2}$ is a hollow endpoint, it belongs to $E_{k}, z_{2}=\varphi_{k}\left(x_{2}\right)$ belongs to $E_{0, k}$ and it separates $z_{1}$ from $z_{2}$.

Construction of $I_{-1, k}$ Ghosts are inserted in $I_{0, k}$ as in Section 4.5. The bipartition $\left(C_{x}, D_{x}\right)$ of $I_{0, k}$ associated to element $x$ in $I_{-1}$ is defined slightly differently. We choose to include in $C_{x}$ (instead of $D_{x}$ ) the set $M(x)$ of elements of $I_{0, k}$ which lie between $C_{x}^{0}$ and $D_{x}^{0}$, i.e., $M(x)=\left\{y \in I_{0, k}: c \succ_{0, k} y \succ_{0, k}\right.$ $\left.d, \forall c \in C_{x}^{0}, d \in D_{x}^{0}\right\}$. We have accordingly:

$$
\begin{aligned}
& C_{x}=M(x) \cup\left\{z \in I_{0, k}: \exists c \in C_{x}^{0} \text { such that } c \succsim_{0, k} z\right\}, \\
& D_{x}=I_{0, k-1} \backslash C_{x}=\left\{y \in I_{0, k}: \exists d \in D_{x}^{0} \text { such that } y \succsim_{0, k} d\right\} .
\end{aligned}
$$

The definition of $\succsim_{-1, k}$ is the same as in Section 4.4 except for Case 3, i.e., when $z_{1}=\varphi_{k}\left(x_{1}\right)$ and $z_{2} \in I_{0, k-1}$. Like in Section 4.4, we have $z_{1} \succ_{-1, k} z_{2}$ if $z_{2} \in D_{x_{1}}$, and $z_{2} \succsim_{-1, k} z_{1}$ if $z_{2} \in C_{x_{1}}$. The only difference is the following. Let $K\left(x_{1}\right)$ denote the set of elements in $I_{-1}$ determining the same bipartition $\left(C_{x_{1}}^{0}, D_{x_{1}}^{0}\right)$ as $x_{1}$. When $z_{2}$ is a least element in $C_{x_{1}}$ (w.r.t. $\succsim_{0, k}$ ) and $x_{1}$ is the greatest element in $K\left(x_{1}\right)$ (w.r.t. $\left.\succsim\right)$, we set $z_{2} \sim_{0, k} z_{1}$.

The proof of Lemma 16 requires adaptation only in Cases 3 and 4 . Let $z_{1}, z_{2} \in I_{0, k}$ be such that $z_{1} \succ_{0, k} z_{2}$.

Case 3 If $z_{1}=\varphi_{-1}\left(x_{1}\right)$ and $z_{2} \in I_{0, k}$, we have that $z_{1} \succ_{0, k} z_{2}$ implies that $z_{2} \in D_{x_{1}}$. The only subcase that differs from Lemma 16 is when $z_{2}$ is a greatest element in $D_{x_{1}}$ and $x_{1}$ is the least element in $K\left(x_{1}\right)$. By definition (9) of the bipartition, $z_{2} \in D_{x_{1}}$, hence $\left(z_{2}, x_{1}\right)$ is a hollow. Therefore $z_{2} \in E_{-1, k}$ and it trivially separates $z_{1}$ from $z_{2}$. 
Case 4 If $z_{1} \in I_{0, k}$ and $z_{2}=\varphi_{-1}\left(x_{2}\right)$, we have that $z_{1} \succ_{0, k} z_{2}$ implies that $z_{1} \in C_{x_{2}}$. The only subcase that differs from Lemma 16 is when $z_{1}$ is a least element in $C_{x_{2}}$ and $x_{2}$ is the greatest element in $K\left(x_{2}\right)$. By construction, in such a case, $z_{1} \sim_{-1, k} z_{2}=\varphi_{-1}\left(x_{2}\right)$, which contradicts $z_{1} \succ_{-1, k} z_{2}$.

Construction of $I_{-l, k}$ As in Section 4.6, we only deal with the construction of $I_{-l, k}$ starting from $I_{-l+1, k}$. Ghost insertion proceeds as in Section 4.6. The bipartition $\left(C_{x}, D_{x}\right)$ of $I_{-l+1, k}$ associated to element $x$ in $I_{-l}$ is defined slightly differently. We choose to include in $C_{x}$ (instead of $D_{x}$ ) the set $M(x)$ of elements of $I_{-l+1, k}$ which lie between $\varphi_{-l+1} C_{x}^{-l+1}$ and $\varphi_{-l+1}\left(D_{x}^{-l+1}\right)$, i.e., $M(x)=\left\{y \in I_{-l+1, k}: c \succ_{-l+1, k} y \succ_{-l+1, k} d, \forall c \in C_{x}^{-l+1}, d \in D_{x}^{-l+1}\right\}$. We have accordingly:

$$
\begin{aligned}
& C_{x}=M(x) \cup\left\{z \in I_{-l+1, k}: \exists c \in \varphi_{-l+1}\left(C_{x}^{-l+1}\right) \text { such that } c \succsim_{-l+1, k} z\right\}, \quad \text { (10) } \\
& D_{x}=I_{-l+1, k} \backslash C_{x}=\left\{y \in I_{-l+1, k}: \exists d \in \varphi_{-l+1}\left(D_{x}^{-l+1}\right) \text { such that } y \succsim_{-l+1, k} d\right\} .
\end{aligned}
$$

The definition of $\succsim_{-l+1, k}$ is the same as in Section 4.6 except for Case 3, i.e., when $z_{1}=\varphi_{-l}\left(x_{1}\right)$ and $z_{2} \in I_{-l+1, k}$. Like in Section 4.6, we have $z_{1} \succ_{-l, k} z_{2}$ iff $z_{2} \in D_{x_{1}}$ and $z_{2} \succ_{-l, k} z_{1}$ iff $z_{2} \in C_{x_{1}}$. The only difference is the following. Let $K\left(x_{1}\right)$ denote the set of elements in $I_{-l}$ determining the same bipartition $\left(C_{x_{1}}^{-l+1}, D_{x_{1}}^{-l+1}\right)$ as $x_{1}$. When $z_{2}$ is a least element in $C_{x_{1}}$ (w.r.t. $\left.\succsim_{-l+1, k}\right)$ and $x_{1}$ is the least element in $K\left(x_{1}\right)$ (w.r.t. $\left.\succsim\right)$, we set $z_{2} \sim_{-l, k} z_{1}$.

The proof of Lemma 16 requires adaptation only in Cases 3 and 4 . Let $z_{1}, z_{2} \in I_{-l, k}$ be such that $z_{1} \succ_{-l, k} z_{2}$.

Case 3 If $z_{1}=\varphi_{-l}\left(x_{1}\right)$ and $z_{2} \in I_{-l+1, k}$, we have that $z_{1} \succ_{0, k} z_{2}$ implies that $z_{2} \in D_{x_{1}}$. The only subcase that differs from Lemma 16 is when $z_{2}$ is a greatest element in $D_{x_{1}}$ and $x_{1}$ is the least element in $K\left(x_{1}\right)$. By definition (10) of the bipartition, $z_{2} \in D_{x_{1}}$, hence $\left(z_{2}, x_{1}\right)$ is a hollow. Therefore $z_{2} \in E_{-l, k}$ and it trivially separates $z_{1}$ from $z_{2}$.

Case 4 If $z_{1} \in I_{-l+1, k}$ and $z_{2}=\varphi_{-l}\left(x_{2}\right)$, we have that $z_{1} \succ_{0, k} z_{2}$ implies that $z_{1} \in C_{x_{2}}$. The only subcase that differs from Lemma 16 is when $z_{1}$ is a least element in $C_{x_{2}}$ and $x_{2}$ is the greatest element in $K\left(x_{2}\right)$. By construction, in such a case, $z_{1} \sim_{-l, k} z_{2}=\varphi_{-l}\left(x_{2}\right)$, which contradicts $z_{1} \succ_{-l, k} z_{2}$.

At the end of this construction process, involving at most a countably infinite number of steps, we obtain, abusing notation, the set $\tilde{I}_{0}=I_{0} \cup\left(\bigcup_{k \geq 0} \varphi_{k}\left(I_{k}\right)\right) \cup$ $\left(\bigcup_{l>0} \varphi_{-l}\left(I_{-l}\right)=I_{0} \cup\left(\bigcup_{m \in M} \varphi_{m}\left(I_{m}\right)\right)\right.$, ordered by $\succsim_{\varphi}$, which is an extension of the complete preorder $\succsim$ on $I_{0}$. It also extends the order $\succsim_{-l, k}$, for all $k,-l \in M$. The 
denumerable set $\tilde{E}_{0}=E_{0} \cup \bigcup_{m \in M} \varphi_{m}\left(E_{m}\right) d$-separates the complete preorder $\succsim_{\varphi}$. Indeed, for all $x \neq y \in \tilde{I}_{0}, x$ and $y$ belong to $I_{-l, k}$ for some $-l, k \in M$ and by Lemma 17, they are separated by some element in $E_{-l, k}$. The complete preorder $\succsim_{\varphi}$ thus admits a numerical representation on the real numbers.

Leaning on this construction, we prove the following proposition, which is the counterpart of Proposition 19.

\section{Proposition 36}

If $f: X \rightarrow] 0,1\left[\subset \mathbb{R}\right.$ is a numerical representation of the complete preorder $\succsim_{\varphi}$ on $\tilde{I}_{0}$, then the function $u$ defined by (6) is a nonstrict unit representation of the semiorder $S=(P, I)$ restricted to $\mathcal{D}$, i.e., for all $x, y \in \mathcal{D}$,

$$
\begin{array}{cll}
u(x) \geq u(y)+1 & \text { iff } & x P y \\
-1<u(x)-u(y)<1 & \text { iff } & x I y
\end{array}
$$

\section{ProOF}

The proof is very similar to that of Proposition 19. We give it for the reader's convenience.

Let $x, y$ be such that $x P y$. If $y$ belongs to $I_{k}(k \in M)$, we have that $x$ belongs to $I_{m}$ for $m \geq k+1$ (by Proposition 13, items 13.4, 13.5 and 13.6). If $x \in I_{k+1}$, we have $u(x)-u(y)=f\left(\varphi_{k+1}(x)\right)+k+1-f\left(\varphi_{k}(y)\right)-k \geq 1$, since $\varphi_{k+1}(x) \succsim_{\varphi} \varphi_{k}(y)$ by construction, and therefore $f\left(\varphi_{k+1}(x)\right) \geq f\left(\varphi_{k}(y)\right)$. Equality occurs when

If $x \in I_{m}$, for $m \geq k+2$, we have $u(x)-u(y)=f\left(\varphi_{m}(x)\right)+m-f\left(\varphi_{k}(y)\right)-k>1$ since $m-k \geq 2$ and $\left|f\left(\varphi_{m}(x)\right)-f\left(\varphi_{k}(y)\right)\right|<1$.

Consider now a pair $x, y \in \mathcal{D}$ such that $x I y$. We assume w.l.o.g. that $x \succ y$ and $y \in I_{k}(k \in M)$. By Proposition 13, items 13.2, 13.3 and 13.5, we know that $x \in I_{k}$ or $x \in I_{k+1}$. In the former case, $0<u(x)-u(y)=f\left(\varphi_{k}(x)\right)+k-f\left(\varphi_{k}(y)\right)-k<1$, since $0<f<1$. In the latter case, we have $0<u(x)-u(y)=f\left(\varphi_{k+1}(x)\right)+k+$ $1-f\left(\varphi_{k}(y)\right)-k<1$ because $f\left(\varphi_{k+1}(x)\right)-f\left(\varphi_{k}(y)\right)<0$. To establish this, we consider the following two possible cases:

- $k \geq 0$. By construction of the ghosts and the extension $\succsim_{\varphi}$ of $\succsim$ for $k \geq 0$, we have $\varphi_{k}(a) \succ_{\varphi} \varphi_{k+1}(x) \succ_{\varphi} \varphi_{k}(b)$ for all $a \in A=\left\{z \in I_{k}: x I z\right\}$ and all $b \in B=\left\{z \in I_{k}: x P z\right\}$. Since $y$ belongs to $A$ and $f$ represents $\succsim_{\varphi}$, we have that $f\left(\varphi_{k}(y)\right)>f\left(\varphi_{k+1}(x)\right)$.

- $k=-l<0$. By construction of the ghosts and the extension $\succsim_{\varphi}$ of $\succsim$ for $k=-l<0$, we have $\varphi_{-l+1}(c) \succ_{\varphi} \varphi_{-l}(y) \succ_{\varphi} \varphi_{-l+1}(d)$ for all $c \in C=\{z \in$ $\left.I_{-l+1}: z P y\right\}$ and all $d \in D=\left\{z \in I_{-l+1}: z I y\right\}$. Since $x$ belongs to $D$ and $f$ represents $\succsim_{\varphi}$, we have that $f\left(\varphi_{-l}(y)\right)=f\left(\varphi_{k}(y)\right)>f\left(\varphi_{-l+1}(x)\right)=$ $f\left(\varphi_{k+1}(x)\right)$. 
To save space, we do not write down explicitly the counterpart of Proposition 21 for I-connected components. It obviously holds and we leave its proof to the reader. The following result states necessary and sufficient conditions for the existence of a nonstrict unit representation of a semiorder. It is a counterpart of Theorem 22 .

\section{Theorem 37}

Let $S=(P, I)$ be a semiorder on the set $X$ and let $\succsim$ be its trace. The following conditions are equivalent.

1. S admits a nonstrict unit numerical representation u satisfying (2);

2. S satisfies the Bounded P-chain condition, the complete preorder $\succsim$ is $d$ separable and $S$ has a denumerable set of hollows;

3. S satisfies the Bounded P-chain condition and is $s^{\prime}$-separable.

The conditions listed in item 2 are independent.

\section{ProOF}

The equivalence of the second and third items results from Proposition 33. The conditions stated in item 2 are necessary for a semiorder to admit a nonstrict unit representation. The necessity of Property 1 and of the $d$-separability of the trace $\succsim$ is shown exactly as for strict representations (see the proof of Theorem 22). A semiorder that has a nonstrict unit representation cannot involve an uncountable set of hollows. This is shown in Remark 34 .

Regarding the sufficiency of these conditions, consider any $I$-connected component $\mathcal{D}$ of the semiorder. If the semiorder has a denumerable set of hollows and its trace is $d$-separable, these properties are inherited by the restrictions of the semiorder and of its trace to $\mathcal{D}$. Therefore, we may apply the construction of $\left(\tilde{I}_{0}, \succsim_{\varphi}\right)$ detailed in the beginning of the present section. Using Proposition 36, we obtain a nonstrict unit representation of the restriction of the semiorder to $\mathcal{D}$. Such a representation can be obtained for each $I$-connected component. Under the Bounded P-chain condition, we know (Bouyssou and Pirlot, 2020a, Remark 64) that it is possible to assemble the unit representations on all connected components into a unit representation of the whole semiorder.

To prove the independence of the three conditions listed in item 2, we slightly modify Examples 23, 24 and 25.

\section{Example 38}

Let $X=\mathbb{R}^{2}$. Consider the binary relation $S$ such that $S=P \cup I$ with $\left(x_{1}, x_{2}\right) P$ $\left(y_{1}, y_{2}\right)$ if $x_{1}>y_{1}+1$ or $\left[x_{1}=y_{1}+1\right.$ and $\left.x_{2} \geq y_{2}\right]$, while $I$ is the symmetric complement of $P$ (i.e., $x I y \Leftrightarrow \operatorname{Not}[x P y]$ and $\operatorname{Not}\left[\begin{array}{ll}y & P\end{array}\right]$ ). 
The only difference w.r.t. Example 23 is that the nonstrict inequality $x_{2} \geq y_{2}$ replaces the strict inequality $x_{2}>y_{2}$ in the definition of $P$. This results in an empty set of hollows, trivially implying the condition on hollows. One shows as in Example 23 that the Bounded $P$-chain condition holds and that $d$-separability is violated.

\section{Example 39}

Let $S=(P, I)$ be the semiorder on $\mathbb{R}$ defined by $x P y$ if $x>y+1$, while $I$ is the symmetric complement of $P$. This relation satisfies the Bounded $P$-chain condition. Its trace is $\geq$ on $\mathbb{R}$; it is therefore $d$-separable. Every pair $(x, y) \in \mathbb{R}^{2}$ with $x=y+1$ is a hollow, hence $S$ does not satisfy the denumerable hollows condition.

\section{Example 40}

Let $X=\mathbb{N} \cup\{\omega\}$. Consider the binary relation $S$ such that $\omega P x$, for all $x \in \mathbb{N}$ and $x P y$ iff $x \geq y+1$, for all $x, y \in \mathbb{N}$, while $I$ is the symmetric complement of $P$. Since $X$ is denumerable, $d$-separability and the condition on hollows trivially hold. The Bounded P-chain condition is violated.

\section{Remark 41}

We proved in preamble of Section 5 that a semiorder that has a nonstrict unit representation need not have a strict unit representation. We are now in a position to prove the converse. Consider for instance the semiorder in Example 39. The set of its hollows is not denumerable. Therefore, by Theorem 37, this semiorder has no nonstrict unit representation.

\section{Remark 42 (Noses-faithful representations)}

In Section 4.9 we showed that all semiorders that admit a strict unit representation also admit a special one in which the endpoints of all hollows are separated by exactly one unit in the representation (hollows-faithful representation). This result has been established by modifiying the ghost insertion procedure leading to the construction of $\left(\tilde{I}_{0}, \succsim_{\varphi}\right)$. This modification consists in placing the ghosts of the hollows in order that they are equivalent w.r.t. $\succsim_{\varphi}$. For any semiorder that admits a nonstrict representation, a similar trick can be used to construct a nonstrict representation in which the values assigned to the endpoints of each nose differ by exactly one unit. We call such a representation noses-faithful. Such a representation is obtained by modifying the construction of $\left(\tilde{I}_{0}, \succsim_{\varphi}\right)$ described in the beginning of Section 5 in the same spirit as what has been done in Section 4.9 for strict representations. For nonstrict representations, the insertion procedure should set the ghosts of noses endpoints as equivalent w.r.t. the $\succsim_{\varphi}$ relation. We leave it to the reader to convince herself that this modification is possible and can be done in a way that preserves the essential properties of $\succsim_{\varphi}$ on $\tilde{I}_{0}$. 


\subsection{Both strict and nonstrict unit representation}

A third sort of numerical representation of a semiorder can be investigated using the tools that we developed to tackle strict unit representations and nonstrict ones.

\section{Definition 43}

A strict-nonstrict unit representation of the semiorder $S=(P, I)$ on the set $X$ is a function $u$ from $X$ to $\mathbb{R}$ such that, for all $x, y \in X$,

$$
\begin{array}{cl}
u(x)>u(y)+1 & \text { if } \quad x P y \\
-1<u(x)-u(y)<1 & \text { if } \quad x I y
\end{array}
$$

With such a representation, the values of two elements of $X$ never differ by exactly one unit.

For semiorders on a denumerable set, it is well-known that they have a strict representation if and only if they have a nonstrict one (Beja and Gilboa, 1992). Moreover, if such a semiorder has a representation (strict or nonstrict), it has a strict-nonstrict one. This is no longer the case with semiorders on an uncountable set.

\section{Proposition 44}

A semiorder on an uncountable set admits a strict-nonstrict representation iff it satisfies the Bounded P-chain condition (Property 1), its trace is d-separable and the number of noses and hollows is at most denumerable.

ProOF

Necessity immediately results from Theorems 22 and 37 since a strict-nonstrict representation is both a strict unit representation and a nonstrict one.

These conditions are also sufficient. This can be proved by adapting the method used for constructing $\left(\tilde{I}_{0}, \succsim_{\varphi}\right)$ for strict unit representations and nonstrict ones. When inserting ghosts of noses or hollows endpoints, one has to make sure that the ghost of any nose (resp. hollow) endpoint and the corresponding other endpoint are never equivalent w.r.t. to the preorder $\succsim_{\varphi}$. It is easy to check that the construction described in Section 4 actually allows to do that in case noses and hollows are denumerable (see also footnote 6, p. 13).

\section{Discussion}

\subsection{Summary}

In this paper we have investigated the conditions under which a semiorder on a set of any cardinality admits a unit representation, either strict or nonstrict. 
We have exhibited a set of three independent conditions that are necessary and sufficient for the existence of a strict unit representation of a semiorder. This has been achieved by factorizing $s$-separability into $d$-separability and the condition that the set of noses is denumerable. We feel that these three conditions have a clear interpretation.

The bounded $P$-chain condition deals with the fact that the threshold is constant and positive. As noted in Bouyssou and Pirlot (2020a, Remark 50), it resembles an Archimedean condition. It applies as soon as the set $X$ is infinite, even countably infinite. It is not specific to strict unit representations. It is easy to check that it is also a necessary condition for nonstrict unit representations.

The $d$-separability condition ensures that the trace of the semiorder, which is a complete preorder, has a numerical representation. This is clearly necessary for strict unit representations but is not specific to them. As can be easily checked, $d$-separability is also necessary for nonstrict unit representations.

Our final condition states that the set of noses is denumerable. It is specific to strict unit representations. For obtaining necessary and sufficient conditions guaranteeing the existence of nonstrict unit representations, we keep unchanged the first two conditions used in the case of strict representations. The third condition is obtained by replacing our condition on "noses" by a condition on the dual notion of "hollows", in a very natural way.

Our results are linked to the discussion in Candeal and Induráin (2010, Sec. 4, p. 489) of Theorem 4.5 in Beja and Gilboa (1992, p. 439). This theorem asserts that a "Generalized Numerical Representation" (GNR) with $\mathscr{S}$ open exists iff $S$ is a semiorder (for which $\succsim$ is antisymmetric) satisfying $d$-separability and the bounded $P$-chain condition and such that the set of $P$-gap-edge-points is denumerable (see below Definition 8, for a definition of $P$-gap-edge-points).

As noted by Candeal and Induráin (2010), the proof of this result (see Beja and Gilboa, 1992, p. 446-448) refers to "positive threshold GNR in which $\mathscr{S}$ is open". This is tantamount to what we have called a strict unit representation. Hence, Candeal and Induráin (2010) wonder whether Beja and Gilboa (1992) were the first to characterize semiorders having a strict unit representation. They state (p. 489, last par. of 2nd col.) that the result in Beja and Gilboa (1992) should be amended by the addition of a condition stating, in our terms, that the set of all right endpoints of lower-half noses should be denumerable.

Our results allow us to be more specific. It is clear that if $x$ is a $P$-gap-edge-point, there is a $y$ such that $(y, x)$ is a nose or a proper lower-half nose (see Definitions 5 and 8). In other terms, $x$ is the right endpoint of a l-h-nose. A l-h-nose, is either a proper l-h-nose or a nose. Whenever $d$-separability is in force, we do not have to ensure the fact that the set of right endpoints of proper l-h-noses is denumerable (Lemma 9). We only have to require that the set of noses is denumerable, which is 
clearly implied by the requirement that the set of all right endpoints of l-h noses is denumerable: requiring that the set of $P$-gap-edge-points is denumerable therefore implies that the set of right endpoints of noses as well as the set of right endpoints of proper l-h-noses are denumerable. Proposition 10 and Theorem 22 show that this is sufficient to guarantee the existence of a strict unit representation. This condition can be weakened however since, as shown in Lemma 9, $d$-separability implies that the set of proper l-h-noses is denumerable. Hence, our result sharpens Beja and Gilboa's result discussed in Candeal and Induráin (2010, Sec. 4), while ensuring its correctness. To bring our result closer to the one of Beja and Gilboa, we could require that the set of all right endpoints of noses is denumerable instead of requiring that the set of all noses is denumerable. Clearly, these two conditions are equivalent: to the right endpoint of a nose corresponds a unique nose (see Bouyssou and Pirlot, 2020b, Remark 1).

A similar discussion can be written down for nonstrict representations. We have shown that Beja and Gilboa's condition that the set of $I$-upper-edge-points is denumerable can be weakened into our condition that the set of hollows is denumerable. Indeed, an $I$-upper-edge-point is the left endpoint of an upper half-hollow (Definition 31). The latter is either a proper upper half-hollow or a hollow. By Lemma 32, we know that the set of proper upper half hollows is denumerable as soon as the semiorder is $d$-separable. Therefore, we do not need to impose that the set of proper upper half-hollows is denumerable. For a $d$-separable semiorder, as soon as the set of hollows is denumerable, the set of upper half-hollows, hence the set of $I$-upper-edge-points, is also denumerable. The similarity with the discussion of the case of strict representations can be made complete. We formulated a condition in the spirit of Candeal and Induráin's $s$-separability condition, which is necessary for the existence of nonstrict representations. This $s^{\prime}$-separability condition is equivalent to $d$-separability of the trace and the condition that the set of hollows is denumerable. Its relationship with Beja and Gilboa's condition that the set of $I$-upper-edge-points is denumerable, can be analyzed as we did above for the case of strict representations.

For characterizing the semiorders that admit either strict or nonstrict unit representations, we used the same proof idea. This proof technique is elementary (we only rely on the existence of numerical representations for complete preorders that are $d$-separable) and constructive (at least in the case of finite semiorders, the ghost construction can be actually implemented to build numerical representations (see Bouyssou and Pirlot, 2020a, Remark 39). It is also unifying. Variants of the same proof technique allow to deal with uncountable and denumerable semiorders, and with the case of strict and nonstrict representations. It allows to prove the existence of various types of special representation: hollows-faithful (Section 4.9), noses-faithful (Remark 42) and strict-nonstrict representations (Section 5.3). We 
believe that this proof technique can be fruitful for solving other questions.

\subsection{Some directions for further work}

Let us first observe that we have left open the question of uniqueness of the representation (be it strict or nonstrict) for semiorders on uncountable sets. This is in contrast with what we did for semiorders on denumerable sets. In Bouyssou and Pirlot (2020a), we sought to keep the construction process of a unit representation as general as possible, in order to guarantee that any unit representation can be obtained by this process (see Bouyssou and Pirlot, 2020a, Prop. 46 and Remark 62). This allowed us to have a good control on the degrees of freedom in the set of unit representations of a semiorder on a denumerable set. In the uncountable case, we made an arbitrary choice of a manner of inserting ghosts that guarantees the $d$-separability of the complete preorder defined on the set of ghosts. Since we do not know how to describe all possible manners of inserting ghosts that preserve $d$-separability, we have lost control on the set of all possible strict (and nonstrict) unit representations in the uncountable case. Hence, the question of the uniqueness of a unit representation in the uncountable case remains open.

There is also a number of questions related to previous work by several authors that require further investigation.

Candeal et al. (2012, Th. 4.11) proved that, for a semiorder, s-separability is equivalent to any of a series of conditions guaranteeing separability for interval orders. These conditions were introduced in Oloriz et al. (1998) and further studied in Bosi et al. (2001) (see also the analysis of separability conditions for biorders in Doignon et al. (1984) and Nakamura (2002)). It would be useful to examine these conditions, equivalent to $s$-separablility for semiorders, and see whether some of them, by way of factorization, could lead to sharper characterization results.

A second issue of interest is related to the kind of real interval representations of biorders, interval orders and semiorders introduced and studied by Nakamura (2002). In these representations, each object is associated an interval, but some objects can be assigned an open one while others are assigned a closed one. The author characterizes the biorders, interval orders and semiorders that admit such "mixed" representations. For semiorders, the particular case of unit length mixed representations is not specifically investigated. Therefore, characterizing the semiorders that admit unit length mixed representations is still an open question. In his study of dense threshold structures, Narens (1994) has given a characterization of semiorders that are isomorphic to the classical semiorder on the reals or the rationals. More generally, one may want to characterize semiorders that are isomorphic to an interval (bounded or not) of the reals or the rationals, endowed with the restriction of the classical semiorder on the reals. The proof technique 
used in the present paper (and its companion Bouyssou and Pirlot, 2020a) can be adapted to establish characterizations for such semiorders. This will be the topic of a future paper.

We also expect that the same idea of proof could be beneficial for the characterization of the semiorders that admit a continuous unit representation (an issue that has received attention (Campíon et al., 2008, Gensemer, 1987a,b, 1988) but seems not to be completely solved).

\section{References}

F. Aleskerov, D. Bouyssou, and B. Monjardet. Utility maximization, choice and preference. Studies in Economic Theory, Volume 16. Springer-Verlag, Berlin, 2nd edition, 2007.

B. Balof, J.-P. Doignon, and S. Fiorini. The representation polyhedron of a semiorder. Order, 30(1):103-135, Mar 2013.

A. F. Beardon, J. C. Candeal, G. Herden, E. Induráin, and G. B. Mehta. The nonexistence of a utility function and the structure of non-representatble preference relations. Journal of Mathematical Economics, 37(1):17-38, 2002.

A. Beja and I. Gilboa. Numerical representation of imperfectly ordered preferences: a unified geometric exposition. Journal of Mathematical Psychology, 36:426-449, 1992.

B. Bosi, J. C. Candeal, E. Induráin, E. Oloriz, and M. Zudaire. Numerical representations of interval orders. Order, 18(2):171-190, 2001.

D. Bouyssou and M. Pirlot. Unit representation of semiorders. I: Countable sets. Working Paper, CNRS-LAMSADE, Université Paris-Dauphine, France, and University of Mons, Belgium, 2020a.

D. Bouyssou and M. Pirlot. A note on Candeal and Induráin's semiorder separability condition. In G. Bosi, M.J. Campión, J.C. Candeal, and E. Induráin, editors, Mathematical Topics on Representations of Ordered Structures and Utility Theory: Essays in Honor of Professor Ghanshyam B. Mehta, pages 129-141. Springer International Publishing, 2020b.

D. S. Bridges and G. B. Mehta. Representations of Preferences Orderings. Lecture Notes in Economics and Mathematical Systems 422. Springer-Verlag Berlin Heidelberg, 1st edition, 1995.

M. J. Campíon, J. C. Candeal, E. Induráin, and M. Zudaire. Continuous representability of semiorders. Journal of Mathematical Psychology, 52(1):48-54, 2008.

J. C. Candeal and E. Induráin. Semiorders and thresholds of utility discrimination: Solving the Scott-Suppes representability problem. Journal of Mathematical Psychology, 54(6):485-490, 2010.

J. C. Candeal, A. Estevan, J. Gutiérrez García, and E. Induráin. Semiorders with separability properties. Journal of Mathematical Psychology, 56:444-451, 2012. doi: 10.1016/j.jmp.2013.01.003. 
J.-P. Doignon. Sur les représentations minimales des semiordres et des ordres d'intervalles. Mathématiques et Sciences humaines, 101:49-59, 1988. URL http: //www. numdam. org/item/MSH_1988_-101_49_0.

J.-P. Doignon, A. Ducamp, and J.-C. Falmagne. On realizable biorders and the biorder dimension of a relation. Journal of Mathematical Psychology, 28(1):73-109, 1984.

S. H. Gensemer. On relationships between numerical representations of interval orders and semiorders. Journal of Economic Theory, 43:157-169, 1987a.

S. H. Gensemer. Continuous semiorder representations. Journal of Mathematical Economics, 16(3):275-289, 1987b.

S. H. Gensemer. On numerical representations of semiorders. Mathematical Social Sciences, 15(3):277-286, 1988.

Alfio Giarlotta and Stephen Watson. Universal semiorders. Journal of Mathematical Psychology, 73:80-93, 2016.

D.H. Krantz, R.D. Luce, P. Suppes, and A. Tversky. Foundations of Measurement, volume 1: Additive and Polynomial Representations. Academic Press, New York, 1971.

R. D. Luce. Semiorders and a theory of utility discrimination. Econometrica, 24(2): 178-191, 1956.

K. L. Manders. On JND representations of semiorders. Journal of Mathematical Psychology, 24(3):224-248, 1981.

Y. Nakamura. Real interval representations. Journal of Mathematical Psychology, 46 (2):140-177, 2002.

L. Narens. The measurement theory of dense threshold structures. Journal of Mathematical Psychology, 38(3):301-321, 1994.

E. Oloriz, J. C. Candeal, and E. Induráin. Representability of interval orders. Journal of Economic Theory, 78(1):219-227, 1998.

M. Pirlot. Minimal representation of a semiorder. Theory and Decision, 28:109-141, 1990.

M. Pirlot. Synthetic description of a semiorder. Discrete Applied Mathematics, 31: 299-308, 1991.

M. Pirlot and Ph. Vincke. Semiorders: properties, representations, applications. Kluwer Academic, 1997.

F. S. Roberts and Ch. H. Franke. On the theory of uniqueness in measurement. Journal of Mathematical Psychology, 14:211-218, 1976.

F.S. Roberts. Measurement theory, with applications to Decision Making, Utility and the Social Sciences. Addison-Wesley, Boston, 1979.

D. Scott and P. Suppes. Foundational aspects of theories of measurement. Journal of Symbolic Logic, 23(2):113-128, 061958. 


\section{Unit representation of semiorders II: The general case}

Denis Bouyssou, Marc Pirlot

\section{To cite this version:}

Denis Bouyssou, Marc Pirlot. Unit representation of semiorders II: The general case. 2020. hal02918017

\section{HAL Id: hal-02918017 https://hal.archives-ouvertes.fr/hal-02918017}

Preprint submitted on 20 Aug 2020

HAL is a multi-disciplinary open access archive for the deposit and dissemination of scientific research documents, whether they are published or not. The documents may come from teaching and research institutions in France or abroad, or from public or private research centers.
L'archive ouverte pluridisciplinaire HAL, est destinée au dépôt et à la diffusion de documents scientifiques de niveau recherche, publiés ou non, émanant des établissements d'enseignement et de recherche français ou étrangers, des laboratoires publics ou privés. 


\title{
Unit representation of semiorders II: The general case
}

\author{
Denis Bouyssou ${ }^{1} \quad$ Marc Pirlot $^{23}$
}

July 14, 2020

${ }^{1}$ LAMSADE, UMR 7243, CNRS, Université Paris-Dauphine, PSL Research University, 75016 Paris, France, e-mail: denis.bouyssou@lamsade.dauphine.fr

${ }^{2}$ Université de Mons, rue de Houdain 9, 7000 Mons, Belgium, e-mail: marc.pirlot@umons.ac.be

${ }^{3}$ Corresponding author 


\begin{abstract}
Necessary and sufficient conditions under which semiorders on uncountable sets can be represented by a real-valued function and a constant threshold are known. We show that the proof strategy that we used for constructing representations in the case of denumerable semiorders can be adapted to the uncountable case. We use it to give an alternative proof of the existence of strict unit representations. A direct adaptation of the same strategy allows us to prove a characterization of the semiorders that admit a nonstrict representation.
\end{abstract}

Keywords: semiorder, numerical representation, constant threshold, uncountable sets 


\section{Contents}

1 Introduction 1

2 Notation and previous results 3

2.1 Notation and definitions . . . . . . . . . . . . . . . 3

2.2 Candeal and Induráin's (2010) result . . . . . . . . . . . . . . . 5

$\begin{array}{lll}3 & \text { Another formulation of } s \text {-separability } & \mathbf{6}\end{array}$

3.1 Noses and hollows . . . . . . . . . . . . . . . . . . 6

3.2 A reformulation of $s$-separability . . . . . . . . . . . 8

4 Semiorders admitting a strict unit representation 9

4.1 Results on the partition into maximal indifference classes . . . . . . 10

4.2 Notation . . . . . . . . . . . . . . . . . . . . 11

4.3 Construction of $I_{0,1} \ldots \ldots \ldots \ldots \ldots$

4.4 Construction of $I_{0, k} \ldots \ldots \ldots \ldots \ldots$

4.5 Construction of $I_{-1, k} \ldots \ldots \ldots \ldots \ldots \ldots \ldots$

4.6 Construction of $I_{-l, k} \quad \ldots \ldots \ldots \ldots \ldots$

4.7 Construction of a representation on an $I$-connected component . . . 23

4.8 Assembling representations on $I$-connected components . . . . . . . 25

4.9 Hollows-faithful representations . . . . . . . . . . . . . . . . 26

4.10 Summary of results . . . . . . . . . . . . . . . . . . . . 33

5 Nonstrict unit representations $\quad 33$

5.1 Separability for nonstrict unit representations . . . . . . . . . . . 34

5.2 Characterization of the semiorders admitting a nonstrict unit representation . . . . . . . . . . . . . . . 37

5.3 Both strict and nonstrict unit representation . . . . . . . . . . . 44

$\begin{array}{llr}6 & \text { Discussion } & 44\end{array}$

6.1 Summary ............................. 44

6.2 Some directions for further work . . . . . . . . . . . . . . 47 


\section{Introduction}

Semiorders were introduced as a way of representing stimuli intensities as they are perceived by a subject. A stimulus is not perceived as more intense than another, unless it is more intense by at least some minimal value called just noticeable difference (JND). The relation on a set of stimuli, comparing their intensities, has the peculiarity that indifference (i.e., stimuli indistinctness) may be intransitive. Luce (1956) defined and studied relations allowing to capture such comparisons, that he called semiorders.

All such relations, provided they are defined on finite sets of objects (e.g., stimuli), admit a numerical representation using real numbers and a positive constant threshold. This constant threshold is closely related to the just noticeable difference. More recently, authors have considered semiorders on denumerable (i.e., finite or countably infinite) and on uncountable sets of objects, respectively. Not all semiorders on infinite sets can be represented by a value function and a constant threshold. Manders (1981) and Beja and Gilboa (1992) have given an additional necessary and sufficient condition guaranteeing that semiorders on denumerable sets can be represented in this way. Bouyssou and Pirlot (2020a) give an alternative proof of this result. The latter proof is, in a sense, constructive. It relies on a double decomposition of the set of objects. This set is first decomposed into connected components of the indifference relation. Second, each connected component is partitioned into maximal indifference classes. One of these classes is selected as a "baseline" in which a representative (which we call "ghost") of each other object is inserted in an appropriate order. Finally, using a numerical representation of the order on the baseline, we construct a numerical representation of the semiorder with unit threshold. In the present paper, we follow the same ideas of proof to deal with uncountable sets of objects ${ }^{1}$.

There are actually (at least) two inequivalent ways of defining constant threshold real valued numerical representations of semiorders. Scott and Suppes (1958) consider numerical representations $(u, k)$ consisting of a real-valued function $u$ defined on the set of objects and a constant threshold $k>0$. The fact that stimulus $x$ is definitely perceived as more intense than stimulus $y$ is represented by the following strict inequality: $u(x)>u(y)+k$. The threshold $k$ can thus be interpreted as the largest unnoticeable difference. Alternatively, it can be decided to represent the same fact by the nonstrict inequality: $u(x) \geq u(y)+k$. Here $k$ can be interpreted as the least noticeable difference. We shall call the former type of representation strict; the latter shall be called nonstrict. While the existence of one type of representation implies that of the other type for semiorders on finite

\footnotetext{
${ }^{1}$ We emphasize that the present paper is self-contained and can be read independently of the one dealing with denumerable semiorders.
} 
sets (Pirlot and Vincke, 1997, p. 72) and countably infinite sets (Beja and Gilboa, 1992, Th. 3.8, p. 436), this is no longer the case for semiorders on uncountable sets.

In the general case, two different sets of necessary and sufficient conditions for the existence of a strict representation (also called a Scott-Suppes representation) have been established by Beja and Gilboa (1992) and by Candeal and Induráin (2010) (see also Giarlotta and Watson, 2016, who derive Candeal and Induráin's result from of a more general analysis). Both characterizations have in common a condition that guarantees the existence of a strict representation in the denumerable case (we call it the Bounded P-chain condition below). On the top of it, Candeal and Induráin impose a new separability condition that they name $s$-separability. In this work, we factorize the $s$-separability condition into the usual Debreu-separability of the trace (i.e., of the complete preorder induced by the semiorder on the set of objects) and another condition that is easily interpretable. This analysis clarifies the relationships between Candeal and Induráin's and Beja and Gilboa's characterizations. Then, using the same strategy of proof as in Bouyssou and Pirlot (2020a), we give an alternative proof of Candeal and Induráin (2010)'s result. We shall discuss later the interest of this method.

Semiorders on uncountable sets that admit a nonstrict representation have been characterized by Beja and Gilboa (1992) (while Candeal and Induráin (2010) did not tackle this question). We establish another characterization, relying on the same proof strategy as before, using a condition similar to $s$-separability. The new type of separability can also be factorized into Debreu-separability of the trace and another easily interpretable condition. The latter also clarifies the relationships with Beja and Gilboa's characterization. Note that the conditions that have to be added to Debreu-separability for obtaining either strict or nonstrict representations use the notions of noses and hollows that were fruitful in the study of finite semiorders (Balof et al., 2013, Doignon, 1988, Pirlot, 1990, 1991).

The paper is organized as follows. In the next section, we introduce the notation and recall the characterization by Candeal and Induráin (2010). In Section 3, we factorize one of the conditions used by Candeal and Induráin (2010) in their characterization theorem into simpler ones. In Section 4, we give an alternative proof of the existence of a strict unit representation for semiorders under three simple conditions. These are necessary and sufficient. We do the same in Section 5 for semiorders admitting nonstrict unit representations. We conclude with a discussion. 


\section{Notation and previous results}

\subsection{Notation and definitions}

We use the same notation as in Bouyssou and Pirlot (2020a). Let $S$ be a binary relation on a set $X$. The relation $S$ is a semiorder if it is complete ${ }^{2}(x S y$ or $y S x$, for all $x, y \in X)$, Ferrers $(x S y$ and $z S w \Rightarrow x S w$ or $z S y$, for all $x, y, z, w \in X$ ) and semi-transitive $(x S y$ and $y S z \Rightarrow x S w$ or $w S z$, for all $x, y, z, w \in X)$. In the sequel, we shall often write the semiorder $S$ as a pair $(P, I)$ of relations, where $P$ (resp. $I$ ) denotes the asymmetric (resp. symmetric) part of $S . P$ is a partial order on $X$, i.e., an asymmetric and transitive relation, which is also Ferrers and semitransitive. $I$ is called the indifference relation; it is reflexive and symmetric but not necessarily transitive. A complete preorder on $X$ is a complete, reflexive, and transitive relation. It is a particular case of a semiorder. A linear order (or total order) on $X$ is a complete, antisymmetric and transitive relation.

The trace $\succsim_{S}$ of a semiorder $S$ on $X$ is the relation defined as follows: for all $x, y \in X, x \succsim_{S} y$ if for all $z \in X, y S z$ implies $x S z$ and $z S x$ implies $z S y$. The subscript $S$ will be omitted whenever there is no ambiguity regarding the associated semiorder. We use $\succ, \precsim, \prec, \sim$ as is usual. It is well-known that the trace of a semiorder is a complete preorder. Two elements $x, y \in X$ such that $x \succsim y$ and $y \succsim x$, i.e., $x \sim y$, are said to be equivalent. If $x \sim y$, then, for all $z \in X$, we have $z S x$ iff $z S y$ and $x S z$ iff $y S z$.

Definition 1 (Strict and nonstrict unit representations)

A strict unit representation of the semiorder $S=(P, I)$ on the set $X$ is a function $u$ from $X$ to $\mathbb{R}$ such that, for all $x, y \in X$,

$$
\begin{array}{cl}
u(x)>u(y)+1 & \text { if } \quad x P y \\
-1 \leq u(x)-u(y) \leq 1 & \text { if } \quad x I y
\end{array}
$$

A nonstrict unit representation of the semiorder $S=(P, I)$ on the set $X$ is a function $u$ from $X$ to $\mathbb{R}$ such that, for all $x, y \in X$,

$$
\begin{array}{cl}
u(x) \geq u(y)+1 & \text { if } \quad x P y \\
-1<u(x)-u(y)<1 & \text { if } \quad x I y
\end{array}
$$

Strict unit representations are the special case of the Scott-Suppes representations (Candeal and Induráin, 2010), in which $k=1$. When dealing with the existence of a Scott-Suppes representation, it is not restrictive to focus on strict unit representations because the latter exists iff the former exists. Nonstrict unit representations

\footnotetext{
${ }^{2}$ Imposing that $S$ is reflexive, instead of complete, would suffice since relations that are both reflexive and Ferrers are complete.
} 
have been less studied (Beja and Gilboa, 1992, is an exception), probably because, a semiorder defined on a denumerable set admits a strict representation iff it admits a nonstrict representation. This is no longer the case in the uncountable case as will be shown in Section 5. In contrast, for other ordered structures, the conditions for the existence of nonstrict representations are well-known. It is the case for biorders (Aleskerov et al., 2007, Beja and Gilboa, 1992, Doignon et al., 1984) and for interval orders (see Aleskerov et al., 2007, Ch. 6).

We shall only consider strict and nonstrict representations that assign the same value to equivalent elements of $X$ w.r.t. the trace $\succsim$ (i.e., regular representations, Roberts and Franke, 1976). Consequently, we may assume henceforth that the equivalence class of each element of $X$ w.r.t. the trace of the semiorder is reduced to a singleton. In other words, for all $x, y \in X, x \succsim y$ and $y \succsim x$ imply $x=y$. This assumption can be made w.l.o.g. (Candeal and Induráin, 2010, Lemma 3.2). Therefore, the trace $\succsim$ is a linear order on $X$. Its asymmetric part is denoted by $\succ$ and its symmetric part by $\sim$.

In Bouyssou and Pirlot (2020a, Section 2.2) (see also Manders, 1981), we have shown that every semiorder $S=(P, I)$, be it on a denumerable or uncountable set, can be decomposed into connected components of the indifference relation $I$. An $I$-connected component of $(X, S)$ is a maximal subset $Y$ of $X$ such that, for each pair $x, y \in Y$, there is an $I$-chain joining them, i.e., there are $x_{1}, x_{2}, \ldots, x_{n} \in$ $Y$ such that $x I x_{1} I x_{2} I \ldots I x_{n} I y$. Furthermore, the relation $P$ induces a linear order (called "macro-ordering" by Giarlotta and Watson (2016)) on the set of $I$-connected components of $(X, S)$.

A necessary condition for the existence of a strict or a nonstrict unit representation of a semiorder is the Bounded P-chain condition (see Property 1 below). Before stating it, we need some definitions.

A $P$-chain is a family of elements $\left(x_{j}, j \in J\right)$ indexed by a set $J \subseteq \mathbb{Z}$ of consecutive integers, such that $x_{j} P x_{j+1}$, for all $j \in J$ with $j+1 \in J$. The $P$-chain is bounded if there are elements $a$ and $b$ such that $b P x_{j} P a$, for all $j \in J$.

\section{Property 1 (Bounded $\boldsymbol{P}$-chain condition)}

Every bounded P-chain is finite.

The semiorder $S$ on the set $X$ satisfies the Bounded $P$-chain condition if every $P$ chain $\left(x_{j}, j \in J\right)$ ( $J$ a set of consecutive integers), that is contained in an interval $[a, b]$ of $(X, \succsim)$ has only finitely many elements $(|J|<\infty)$ (Bouyssou and Pirlot, 2020a, Cor. 53).

Bouyssou and Pirlot (2020a, Proposition 54) establishes that the Bounded Pchain condition is equivalent to the necessary and sufficient condition of Manders (1981) for the existence of a unit representation of a semiorder on a denumerable set. The Bounded P-chain condition is also equivalent to Beja and Gilboa 
(1992)'s condition (Bouyssou and Pirlot, 2020a, Sections 5.2 and 5.3). Candeal and Induráin (2010) call this property regularity ${ }^{3}$. If $X$ is $I$-connected, it is clear that $(X, S)$ always satisfies the Bounded $P$-chain condition (Bouyssou and Pirlot, 2020a, Proposition 54).

The strategy followed in Bouyssou and Pirlot (2020a) to show the existence of a unit representation for a semiorder defined on a denumerable set is to show the existence of such a representation on each $I$-connected component of the semiorder and then, assuming the Bounded P-chain condition, to show that it is possible to assemble these representations into a representation of the whole semiorder. We shall adopt the same strategy in case the semiorder is defined on an uncountable set. Under an additional condition, we show that a strict unit representation exists on each $I$-connected component and we assemble these representations assuming the Bounded $P$-chain condition ${ }^{4}$. This can be done also for nonstrict unit representations by substituting the additional condition for strict representations by an additional condition that is suitable for nonstrict representations.

In the next subsection, we recall Candeal and Induráin's (2010) result and the additional condition that they have to impose on semiorders in order to prove the existence of a strict unit representation.

\subsection{Candeal and Induráin's (2010) result}

Candeal and Induráin (2010) assume the following additional condition that they call $s$-separability.

\section{Definition 2 ( $s$-separability)}

A semiorder $S=(P, I)$ on $X$ is s-separable if there is a denumerable set $E, E \subseteq X$, such that, for all $a, b \in X$ with $a P b$, there are

$$
\begin{aligned}
& c \in E \quad \text { such that } a P c \succsim b \\
& \text { and } d \in E \text { such that } a \succsim d P b
\end{aligned}
$$

The main result in (Candeal and Induráin, 2010, Theorem 3.6) can be rephrased as follows.

Theorem 3 (Candeal and Induráin (2010))

$A$ semiorder $S$ on a set $X$ admits a strict unit representation iff it satisfies the Bounded P-chain condition and is s-separable.

\footnotetext{
${ }^{3}$ This regularity property should not be confused with the regularity property of representations introduced by Roberts and Franke (1976)

${ }^{4}$ As observed in Bouyssou and Pirlot (2020a, Remark 64) assembling representations on $I$ connected components of a semiorder is possible for uncountable semiorders, under the same conditions as for denumerable semiorders.
} 
Notice that the trace of an $s$-separable semiorder is $d$-separable or Debreu-separable (Candeal and Induráin, 2010, Lemma 3.4), which is a condition guaranteeing the existence of a numerical representation of the trace (i.e., the existence of a function $v: X \rightarrow \mathbb{R}$ such that $x \succsim y$ iff $v(x) \geq v(y)$ ). It can be assumed w.l.o.g. that a representation of the semiorder also represents its trace. Indeed, a numerical representation $u$ of the semiorder must give distinct values to elements that are not equivalent w.r.t. the trace. Therefore $u$ must represent the trace since we assumed that no elements are equivalent w.r.t. the trace. We recall the definition of Debreu-separability below.

\section{Definition 4 ( $d$-separability)}

A semiorder $S=(P, I)$ is d-separable if its trace $\succsim i s d$-separable. The trace is $d$-separable if it admits a denumerable order-dense set, i.e., there is a denumerable set $D \subseteq X$, such that, for all $a, b \in X$ with $a \succ b$, there is $d \in D$, such that $a \succsim d \succsim b$.

The $d$-separability of the trace is a necessary condition for the existence of both a strict and a nonstrict (see Section 5) representation of a semiorder.

\section{Another formulation of $s$-separability}

In this section we revisit the $s$-separability condition and factorize it into $d$ separability and another condition. The latter is expressed in terms of noses, a notion introduced in Pirlot $(1990,1991)$, together with that of hollows. These have proved useful in the study of the unit representations of finite semiorders (Balof et al., 2013, Doignon, 1988, Pirlot, 1990, 1991).

The results in this section have appeared in Section 3.2 of Bouyssou and Pirlot (2020b). For the ease of reading, we recall them here.

\subsection{Noses and hollows}

Let $S=(P, I)$ be a semiorder on $X$. A nose of $S$ is a pair $(a, b)$ such that $a P b$ and $a$ is the least element (w.r.t. $\succ$ ) which is preferred to $b$ and $b$ is the greatest element (w.r.t. $\succ$ ) which $a$ is preferred to. A hollow is a pair $(a, b)$, with $a I b$ and $a \succsim b$ such that $a$ is the greatest element (w.r.t. $\succ$ ) which is indifferent to $b$ and $b$ is the least element (w.r.t. $\succ$ ) which is indifferent to $a$. More formally, we have:

\section{Definition 5 (Noses and Hollows)}

The ordered pair $(a, b) \in X \times X$ is a nose of $S=(P, I)$ if $a P b$ and there is no $c \in X$ such that $a P c \succ b$ and there is no $d \in X$ such that $a \succ d P b$.

The ordered pair $(a, b) \in X \times X$ is a hollow of $S=(P, I)$ if $a I b, a \succ b$, and there is no $c \in X$ such that $b I c \succ a$ and there is no $d \in X$ such that $b \succ d I a$. 


\section{Remark 6}

A hollow can be equivalently defined in terms of the relation $S$. The pair $(a, b)$, with $a \succ b$, is a hollow iff $b S a$, for all $d \prec b, \operatorname{Not}[d S a]$, and for all $c \succ a, \operatorname{Not}[b S c]$. Indeed, since $a \succ b$, there is $c$ with $c \succ a$ and $b I c$ iff there is $c$ with $\succ a$ and $b S c$; likewise, there is $d$ with $b \succ d$ and $d I a$ iff there is $d$ with $b \succ d$ and $d S a$.

Noses play a special role w.r.t. $s$-separability as shown by the following lemma.

\section{Lemma 7}

If the semiorder $S=(P, I)$ on $X$ is s-separable by the denumerable set $E$, then a and $b$ belong to $E$ whenever $(a, b)$ is a nose.

\section{PROOF}

Let $(a, b)$ be a nose, so that $a P b$. By the $s$-separability property, there is $c \in E$ such that $a P c \succsim b$. By definition of a nose, we have $c=b$ and therefore, $b \in E$. Using $s$-separability, there is also $d \in E$ such that $a \succsim d P b$, which implies $a=d$ and $a \in E$ since $(a, b)$ is a nose.

Before presenting conditions equivalent to $s$-separability, we need to establish some auxiliary results related to variants of noses.

\section{Definition 8 (Half-noses)}

The pair $(a, b) \in X \times X$ is a lower half-nose (l-h-nose) of $S=(P, I)$ if aPb and there is no $c \in X$ such that $a P c \succ b$. The pair $(a, b)$ can be a nose. If it is not, we say it is a proper l-h-nose if it is a l-h-nose and there is $d \in X$ such that $a \succ d P b$. The pair $(a, b) \in X \times X$ is an upper half-nose (u-h-nose) of $S=(P, I)$ if aPb and there is no $d \in X$ such that $a \succ d P b$. The pair $(a, b)$ can be a nose. If it is not, we say it is a proper $u$-h-nose if it is an $u$-h-nose and there is $c \in X$ such that $a P c \succ b$. We denote by LHN (resp. UHN) the set of right endpoints b (resp. left endpoints a) of all proper l-h-noses (resp. u-h-noses) $(a, b)$.

The notion of lower half-nose is closely related to that of $P$-gap-edge point (Beja and Gilboa, 1992, Definition P6 (a), p. 438). The element $b \in X$ is a $P$-gap-edgepoint if and only if there is $a \in X$ such that $(a, b)$ is a lower half-nose. We have the following result.

\section{Lemma 9}

If the semiorder $S=(P, I)$ is d-separable, then the sets $L H N$ and $U H N$ are denumerable.

\section{PROOF}

We give the proof for $L H N$. The case of $U H N$ is similar.

Let $(a, b)$ be a proper l-h-nose. We define the set $N(b)=\{x \in X: x P b$ and $\forall c \succ$ $b, N o t[x P c]\}$. In other words, for all $x \in N(b),(x, b)$ is a l-h-nose. It is clear 
that $a \in N(b)$. Moreover, since $(a, b)$ is proper, $N(b)$ contains at least one other element $d$ such that $a \succ d P b$. We now prove that $N(b)$ is an interval w.r.t. $\succ$. Let $x, x^{\prime} \in N(b)$. If $x^{\prime \prime}$ is such that $x \succ x^{\prime \prime} \succ x^{\prime}$, then $x^{\prime \prime} \in N(b)$. Indeed, $x^{\prime \prime} P b$ since $x^{\prime} P b$ and for all $c \succ b, \operatorname{Not}\left[x^{\prime \prime} P c\right]$ since Not $[x P c]$. Let $(a, b)$ and $\left(a^{\prime}, b^{\prime}\right)$ be two proper l-h-noses, with $b \neq b^{\prime} \in L H N$. It is clear that the associated intervals $N(b)$ and $N\left(b^{\prime}\right)$ are disjoint. Each of these intervals contains at least two distinct points and therefore at least an element from the denumerable set $D$ that $d$-separates $S=(P, I)$. Consequently, the set $L H N$ is denumerable.

\subsection{A reformulation of $s$-separability}

We are in position to prove an equivalent formulation for $s$-separability.

\section{Proposition 10}

A semiorder $S=(P, I)$ on $X$ is s-separable iff $\succsim$ is $d$-separable and the set of noses is denumerable.

\section{PROOF}

Assume that the semiorder is $s$-separable. By Lemma 7 , the set of noses is denumerable. The $s$-separability property implies that $\succsim$ is $d$-separable (Candeal and Induráin, 2010, Lemma 3.4). We include the proof of this for completeness. Let $x, y \in X$ be such that $x \succ y$. There is $z \in X$ such that $x P z$ and $z S y$ and/or $w \in X$ such that $w P y$ and $x S w$. In the former case, $s$-separability entails that there is $d \in E$ such that $x \succsim d P z$ and, since $z S y$, we have $x \succsim d \succ y$. In the latter case, there is $c \in E$ such that $w P c \succsim y$ and, since $x S w$, we have $x \succ c \succsim y$.

Reciprocally, let $D$ be a denumerable set that $d$-separates $\succ$. Let $x, y \in X$ be such that $x P y$. If $(x, y)$ is not a nose,

1. either there is $x^{\prime} \prec x$ such that $x^{\prime} P y$

2. or there is $y^{\prime} \succ y$ such that $x P y^{\prime}$.

In Case 1 , by the $d$-separability of $\succ$, there is $d \in D$ such that $x^{\prime} \precsim d \precsim x$. Therefore we have $x \succsim d P y$. Further, there are two cases. Either there is $y^{\prime} \succ y$ such that $x P y^{\prime}$ or for all $y^{\prime} \succ y$, we have Not $\left[x P y^{\prime}\right]$. In the former case, $d$ separability implies that there is $c \in D$ such that $y^{\prime} \succsim c \succsim y$. Then, we have $x P c \succsim y$. Otherwise, $(x, y)$ is a proper l-h-nose. In order to have $c \in E$ such that $x P c \succsim y$, we set $c=y$ and include the denumerable set $L H N$ of right endpoints of the proper l-h-noses in $E$.

In Case 2, by the $d$-separability of $\succ$, there is $c \in D$ such that $y^{\prime} \succsim c \succsim y$. Therefore we have $x P c \succsim y$. Further, either there is $x^{\prime} \prec x$ such that $x^{\prime} P y$ or for all $x^{\prime} \prec x$, we have $\operatorname{Not}\left[x^{\prime} P y\right]$. In the former case, $d$-separability implies that there is $d \in D$ such that $x^{\prime} \precsim d \precsim x$. Otherwise, $(x, y)$ is a proper $\mathrm{u}$-h-nose. In order 
to have $d \in E$ such that $x \succsim d P y$, we set $d=x$ and include the denumerable set $U H N$ of left endpoints of the proper u-h-noses in $E$.

Finally, by considering $E$ as the union of $D, L H N, U H N$ and the set of elements $a, b$ such that $(a, b)$ is a nose, which is denumerable by hypothesis, we obtain a denumerable set $E$, which $s$-separates the semiorder $(P, I)$.

\section{Remark 11}

It is easy to show that having a denumerable set of noses is a necessary condition for a semiorder to have a strict unit representation. Indeed, assume that $u$ is a strict unit representation of the semiorder $S=(P, I)$ and $(a, b)$ is a nose of $S$. Since $a P b$, we have $u(a)>u(b)+1$. Let $\varepsilon_{a b}$ be the positive number $u(a)-u(b)-1$. By definition of a nose, there is no element $c \neq b$ such that $a P c \succ b$ and therefore, there is no $c$ such that $u(c) \in] u(b), u(a)-1]$, an interval of length $\varepsilon_{a b}>0$. To each nose $(a, b)$ is associated such an interval of positive length and all these intervals are disjoint. Since there is only a denumerable number of disjoint intervals of positive length in $\mathbb{R}$, the number of noses is denumerable.

\section{Remark 12}

For proving the existence of a strict unit representation, we shall use $d$-separability and the condition that the number of noses is denumerable, instead of $s$-separability. In the proof, we shall only use the denumerable set $D$ that is dense in the trace $\succsim$ and the denumerable set of noses endpoints. We do not need to add the half-noses or the half-hollows as we had to do in the second part of the proof of Proposition 10. In other words, we do not use all the points in the set $E$ involved in the $s$-separability property (see Definition 2). In the same vein, we do not need to impose that the set of all $P$-gap-edge-points (Beja and Gilboa, 1992, p. 438) is denumerable. Only the cardinality of the set of $P$-gap-edge-points that correspond to noses needs to be controlled. Our condition that the set of noses has to be denumerable refines both the $s$-separability condition and the denumerability of the set of P-gap-edge-points (Beja and Gilboa, 1992, Theorem 4.5 (a), p. 439). Its interpretation is straightforward and it bridges the gap between the conditions imposed by Candeal and Induráin (2010) and those imposed by Beja and Gilboa (1992). A similar analysis will be helpful in the study of nonstrict representations (see Section 5.1).

\section{Semiorders admitting a strict unit representa- tion}

In this section, we adapt the method proposed in Bouyssou and Pirlot (2020a) for building a unit representation to the case of uncountable semiorders. In the denumerable case, we select an initial maximal indifference class $I_{0}$ of an $I$-connected 
component of the semiorder and we insert a "ghost" of each other element of this connected component at an appropriate position in $I_{0}$. The set of all ghosts is denoted $\tilde{I}_{0}$ and $\succsim_{\varphi}$ is a preorder on $\tilde{I}_{0}$ extending the trace on $I_{0}$. This insertion procedure has to be refined in the uncountable case. The main constraint is to insert the ghosts of all elements while ensuring the $d$-separability of the complete preorder $\succsim_{\varphi}$ on the set of ghosts $\tilde{I}_{0}$. Moreover, in contrast with the denumerable case, it is no longer possible to insert ghosts sequentially since $X$ is uncountable. Notice that, unlike in the denumerable case, we shall not attempt to keep the possibility of generating all possible unit representations. We concentrate on proving there is at least one.

\subsection{Results on the partition into maximal indifference classes}

We assume that the semiorder $(X, S)$ has been decomposed into its $I$-connected components. In Bouyssou and Pirlot (2020a, Section 3), we showed that any Iconnected component of a semiorder can be partitioned into maximal indifference classes. This was proved in the general case, without assuming that the semiorder is defined on a denumerable set. Such a partition gives a particular role to a baseline maximal indifference class $I_{0}$. This partition is not unique in general, but the results that follow are valid for any such partition.

Let $\mathcal{D}$ be any $I$-connected component of the semiorder $S=(P, I)$ on the set $X$. We abuse notation also using $S=(P, I)$ for denoting the restriction of the semiorder to the set $\mathcal{D} \subseteq X$. Let $\left(I_{m}, m \in M\right)$, with $M \subseteq \mathbb{Z}, M \ni 0$, be a partition of $\mathcal{D}$ into maximal indifference classes, as described in Bouyssou and Pirlot (2020a, Section 3). We shall also use the more explicit notation $\ldots, I_{-l}, \ldots, I_{-1}, I_{0}$, $I_{1}, \ldots, I_{k}, \ldots$ for this partition, with indices $k, l \geq 0$ such that $k \in M$ and $-l \in M$. Note that the set of indices $M$ may be bounded or unbounded. We recall below Bouyssou and Pirlot (2020a, Proposition 23) that collects useful properties of such a partition.

\section{Proposition 13}

The sets $\left(I_{m}, m \in M\right)$ have the following properties:

1. They are disjoint nonempty convex subsets of $\mathcal{D}$.

2. Their elements are pairwise indifferent, i.e., for all $x, y \in I_{m}$, we have $x I y$.

3. They form an ordered partition w.r.t. $\succ$, i.e. for all $x \in I_{m-1}$ and $z \in I_{m}$, we have $z \succ x$.

4. For all $m \geq 0$ for which $I_{m}$ and $I_{m+1}$ exist, for all $w \in I_{m+1}$, there is $z \in I_{m}$ such that we have $w P z$. 
5. For all $m<0$ for which $I_{m}$ and $I_{m+1}$ exist, for all $v \in I_{m}$, there is $z \in I_{m+1}$ such that we have zPv.

6. For all $m \in M$ for which $I_{m}$ and $I_{m+2}$ exist, for all $w \in I_{m+2}$, for all $v \in I_{m}$, we have $w P v$.

7. $\mathcal{D}=\cup_{m \in M} I_{m}$.

In the process of constructing a unit representation, we make the hypothesis that the trace $\succsim$ of the semiorder $S$ on $X$ is $d$-separable and that the semiorder has a denumerable set of noses. These properties of the semiorder on $X$ are inherited by its restriction to each $I$-connected component. The trace of the restriction of the semiorder to an $I$-connected component is the restriction of the trace of the semiorder to this component (because all elements in an $I$-connected component compare identically to the elements of the other components).

Let $E=E^{\prime} \cup E^{\prime \prime}$ denote the union of $E^{\prime}$, an order-dense denumerable subset w.r.t. $\succsim$, and the set $E^{\prime \prime}$ of all noses endpoints. The set $E$ is thus at most denumerable.

\subsection{Notation}

In order to describe the construction process of a strict unit representation, we need to introduce some notation and definitions.

Ordered bipartitions play an important role in the sequel. Therefore, we recall some precise definitions (following Bridges and Mehta, 1995, p. 17). Let $\succsim$ be a linear order on a set $Y$ and $\succ$ its asymmetric part. We call $(A, B)$ an ordered bipartition of $Y$ if $A \cap B=\varnothing, A \cup B=Y$ and $x \succ y$ for all $x \in A, y \in B$. One of the classes may be empty. An ordered bipartition $(A, B)$, with $A, B \neq \varnothing$,

- is a jump if $A$ has a least element and $B$ has a greatest element;

- is a cut if either $A$ has least element or $B$ has a greatest element (but not both);

- is a gap if neither $A$ has a least element nor $B$ has a greatest element.

Note that, in the absence of ambiguity, we shall write "bipartition" for "ordered bipartition" 5 .

Each element $x \in I_{m}$ determines a bipartition $\left(A_{x}^{m-1}, B_{x}^{m-1}\right)$ of $I_{m-1}$ (provided $I_{m-1}$ exists) and a bipartition $\left(C_{x}^{m+1}, D_{x}^{m+1}\right)$ of $I_{m+1}$ (provided $I_{m+1}$ exists). We have (see also Figure 1:

\footnotetext{
${ }^{5}$ We shall not adopt the term "decomposition", which is used by Bridges and Mehta (1995) as synonymous to "ordered bipartition" because we use "decomposition" for other purposes.
} 
- $A_{x}^{m-1}=\left\{y \in I_{m-1}: x I y\right\}$;

- $B_{x}^{m-1}=\left\{y \in I_{m-1}: x P y\right\}$;

- $C_{x}^{m+1}=\left\{y \in I_{m+1}: y P x\right\}$;

- $D_{x}^{m+1}=\left\{y \in I_{m+1}: y I x\right\}$

For $m>0, B_{x}^{m-1}$ is non-empty; for $m \leq 0, C_{x}^{m+1}$ is non-empty. This follows from Proposition 13.4 and 13.5.

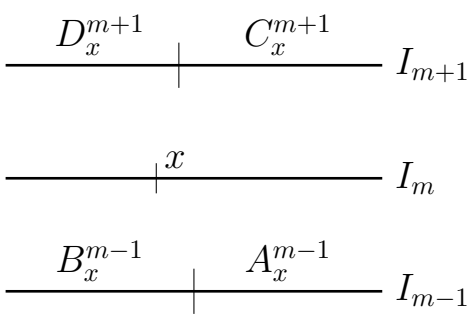

Figure 1: Partitions determined by an element $x \in I_{m}$

The construction of a representation proceeds by selecting a baseline maximal indifference class $I_{0}$ and inserting dummies of the elements of $I_{m}, m \in M \backslash\{0\}$ into $I_{0}$ at an "appropriate location", i.e., by positioning the dummies among the elements of $I_{0}$. In this way, we shall extend the trace $\succsim$ on $I_{0}$ into a complete preorder on $I_{0}$ and the dummies of all other elements in $\mathcal{D}$. We shall also refer to the dummies as ghosts (like in Bouyssou and Pirlot, 2020a). We recall that $E$ denotes a denumerable set that $d$-separates the trace $\succsim$ and contains all noses endpoints. Here is some notation that will be used in the sequel:

$\varphi_{m}\left(I_{m}\right)$, for $m \in M \subseteq \mathbb{Z}$, is the set of ghosts of the elements in $I_{m}$;

$\varphi_{m}(x)$, for $m \in M, x \in I_{m}$, is the ghost of the element $x$ in $I_{m}$;

$\varphi_{0}\left(I_{0}\right)=I_{0} ; \varphi_{0}(x)=x$, for all $x \in I_{0} ;$

$I_{0,1}=I_{0} \cup \varphi_{1}\left(I_{1}\right) ;$

$I_{0, k}=I_{0} \cup \varphi_{1}\left(I_{1}\right) \cup \ldots \cup \varphi_{k}\left(I_{k}\right)$, for $k \in M, k>0$;

$I_{-l, k}=I_{0} \cup \varphi_{1}\left(I_{1}\right) \cup \ldots \cup \varphi_{k}\left(I_{k}\right) \cup \varphi_{-1}\left(I_{-1}\right) \ldots \cup \varphi_{-l}\left(I_{-l}\right)$, for $k, l \in M, k, l>0$;

$E_{m}=E \cap I_{m}$

$E_{0,1}=E_{0} \cup \varphi_{1}\left(E_{1}\right) ;$ 
$E_{0, k}=E_{0} \cup \varphi_{1}\left(E_{1}\right) \cup \ldots \cup \varphi_{k}\left(E_{k}\right)$, for $k \in M, k>0$;

$E_{-l, k}=E_{0} \cup \varphi_{1}\left(E_{1}\right) \cup \ldots \cup \varphi_{k}\left(E_{k}\right) \cup \varphi_{-1}\left(E_{-1}\right) \ldots \cup \varphi_{-l}\left(E_{-l}\right)$, for $k, l \in M, k, l>0$;

$\succsim_{0,1}$ is a complete preorder on $I_{0,1}$, with $\succ_{0,1}$, its asymmetric part, and $\sim_{0,1}$, its symmetric part;

$\succsim_{0, k}$ is a complete preorder on $I_{0, k}$, with $\succ_{0, k}$, its asymmetric part, and $\sim_{0, k}$, its symmetric part, for $k \in M, k>0$;

$\succsim_{-l, k}$ is a complete preorder on $I_{-l, k}$, with $\succ_{-l, k}$, its asymmetric part, and $\sim_{-l, k}$, its symmetric part, for $k, l \in M, k, l>0$.

\subsection{Construction of $I_{0,1}$}

The set $I_{0,1}$ is the union of $I_{0}$ and the set of the ghosts of the elements of $I_{1}$. The ghost $\varphi_{1}(x)$ for $x \in I_{1}$ is distinct from all elements of $I_{0}$. It is defined as being positioned between the two classes of the bipartition $\left(A_{x}^{0}, B_{x}^{0}\right)$, i.e., above all elements in $B_{x}^{0}$ and below all elements in $A_{x}^{0}$. More precisely, we define the relation $\succsim_{0,1}$ on $I_{0,1}$ as an extension of the order $\succsim$ on $I_{0}$ which satisfies $a \succsim_{0,1} \varphi_{1}(x) \succ_{0,1} b$, for all $a \in A_{x}^{0}$ and $b \in B_{x}^{0}$.

In case several elements of $I_{1}$ determine the same bipartition, their ghosts all have to be inserted in between the classes of the bipartition. Let $J(x)$ denote the set of all elements in $I_{1}$ which determine the same bipartition $\left(A_{x}^{0}, B_{x}^{0}\right)$ as $x$. For all $x_{1}, x_{2} \in J(x)$, with $x_{1} \succ x_{2}$, we have $a \succsim_{0,1} \varphi_{1}\left(x_{1}\right) \succ_{0,1} \varphi_{1}\left(x_{2}\right) \succ_{0,1} b$, for all $a \in A_{x}^{0}$ and $b \in B_{x}^{0}$. At this stage, the only case in which we place a ghost $\varphi_{1}\left(x_{1}\right)$ and an element $b$ of $I_{0}$ in the same equivalence class of the relation $\succsim_{0,1}$ is when $\left(x_{1}, a\right)$ is a hollow. In such a case, we set $a \sim_{0,1} \varphi_{1}\left(x_{1}\right)$. Note that we are not forced to make these elements equivalent in all $\operatorname{cases}^{6}$, but we shall systematically choose this option in the rest of this section. We emphasize that $a$ and $\varphi_{1}\left(x_{1}\right)$ are distinct elements in $I_{0,1}$, yet they are equivalent w.r.t. $\sim_{0,1}$.

The relation $\succsim_{0,1}$ just defined has the following properties.

\section{Lemma 14}

$\succsim_{0,1}$ is a preorder on $I_{0,1}$ which extends $\succsim$ on $I_{0}$, satisfies $\varphi_{1}(x) \succ_{0,1} \varphi_{1}(y)$ for all $x, y \in I_{1}$ with $x \succ y$, and is $d$-separable using $E_{0,1}$.

\footnotetext{
${ }^{6}$ In case $\left(x_{1}, a\right)$ is a hollow (with $x_{1} \in I_{1}$ and $a \in I_{0}$ ), we may decide to set $a \succ_{0,1} \varphi_{1}\left(x_{1}\right)$ instead of $\varphi_{1}\left(x_{1}\right) \sim_{0,1} a$. In such a case, $a$ and $x_{1}$ have to be added to $E_{0,1}$. The latter option can be taken as long as it preserves the denumerable character of the separating set, i.e., for at most a denumerable set of hollows $\left(x_{1}, a\right)$.
} 


\section{PROOF}

The fact that relation $\succsim_{0,1}$ is complete and transitive is an easy consequence of the corresponding properties of $\succsim$ and of the construction of $I_{0,1}$. By construction, we have that $\succsim_{0,1}$ is an extension of $\succsim_{0} I_{0}$, i.e., for all $a, b \in I_{0}, a \succsim_{0,1} b$ iff $a \succsim b$ and that the bijection $\varphi_{1}$ mapping $I_{1}$ onto $\varphi_{1}\left(I_{1}\right)$ respects the order induced by $\succsim$ on $I_{1}$, i.e., for all $x, y \in I_{1}, x \succ y$ iff $\varphi_{1}(x) \succ_{0,1} \varphi_{1}(y)$. The fact that relation $\succsim_{0,1}$ is complete and transitive is an easy consequence of the corresponding properties of $\succsim$ and of the construction of $I_{0,1}$.

The relation $\succsim_{0,1}$ is $d$-separable. Let $z_{1} \succ_{0,1} z_{2}$ with $z_{1}, z_{2} \in I_{0,1}$. Four cases can be distinguished.

Case 1 If $z_{1}, z_{2} \in I_{0}$ then we have $z_{1} \succ_{0,1} z_{2}$ iff $z_{1} \succ z_{2}$. These $z_{1}$ and $z_{2}$ can be separated by an element from $E_{0}$.

Case 2 If $z_{1}=\varphi_{1}\left(x_{1}\right)$ and $z_{2}=\varphi_{1}\left(x_{2}\right)$ for some $x_{1}, x_{2} \in I_{1}$, we know that $z_{1} \succ_{0,1} z_{2}$ implies $x_{1} \succ x_{2}$. Since $\succsim$ is $d$-separable, there is an element in $E_{1}$ separating $x_{1}$ from $x_{2}$. Therefore, $z_{1}$ and $z_{2}$ are separated by an element in $\varphi_{1}\left(E_{1}\right)$.

Case 3 If $z_{1}=\varphi_{1}\left(x_{1}\right)$ and $z_{2} \in I_{0}$, we have that $z_{1} \succ_{0,1} z_{2}$ implies $z_{2} \in B_{x_{1}}^{0}$. If $z_{2}$ is not the greatest element in $B_{x_{1}}^{0}$ (w.r.t. $\succ$ ), then there is $z \in B_{x_{1}}^{0}$ such that $z_{1} \succ_{0,1} z \succ z_{2}$. By hypothesis, $z_{2}$ can be separated from $z$, and therefore from $z_{1}$ by an element from $E_{0}$. If $z_{2}$ is the greatest element in $B_{x_{1}}^{0}$ and there is $x \in J\left(x_{1}\right)$ with $x_{1} \succ x$, then $\varphi_{k}\left(x_{1}\right) \succ_{0,1} \varphi_{k}(x) \succ_{0,1} z_{2}$ and we can use the separability of $\succsim$. Therefore, $z_{1}$ and $z_{2}$ are separated by an element in $\varphi_{1}\left(E_{1}\right)$. Finally, we consider the case in which $z_{2}$ is the greatest element in $B_{x_{1}}^{0}$ and $x_{1}$ is the least element in $J\left(x_{1}\right)$. In such a case, $\left(x_{1}, z_{2}\right)$ is a nose. Since $z_{2}$ is a nose endpoint, it belongs to $E_{0}$ and it separates $z_{1}$ from $z_{2}$.

Case 4 If $z_{1} \in I_{0}$ and $z_{2}=\varphi_{1}\left(x_{2}\right)$, we have that $z_{1} \succ_{0,1} z_{2}$ implies that $z_{1} \in A_{x_{2}}^{0}$. If $z_{1}$ is not the least element in $A_{x_{2}}^{0}$ (w.r.t. $\succsim$ ), then there is $z \in A_{x_{2}}^{0}$ such that $z_{1} \succ z \succ_{0,1} z_{2}$. By hypothesis, $z_{1}$ can be separated from $z$, and therefore from $z_{2}$, by an element from $E_{0}$. If $z_{1}$ is the least element in $A_{x_{2}}^{0}$ and there is $x \in J\left(x_{2}\right)$ such that $x \succ x_{2}$, then $z_{1} \succsim_{0,1} \varphi_{1}(x) \succ_{0,1} \varphi_{1}\left(x_{2}\right)$. Using the separability of $\succsim$, we can separate $z_{1}$ and $z_{2}$ by an element in $\varphi_{1}\left(E_{1}\right)$. Finally, the case in which $z_{1}$ is the least element in $A_{x_{2}}^{0}$ and $x_{2}$ is the greatest element such that $z_{1} \succsim_{0,1} \varphi_{1}\left(x_{2}\right)$ does not occur since this would mean that $\left(x_{2}, z_{1}\right)$ is a hollow; in such a case, by construction, $\varphi_{1}\left(x_{2}\right) \sim_{0,1} z_{1}$ (note that, in the next steps, this situation will not necessarily occur for hollows). 


\subsection{Construction of $I_{0, k}$}

We assume that $I_{0, k-1}$ has been constructed as well as the complete preorder $\succsim_{0, k-1}$ on it. The set $I_{0, k}$ is the union of $I_{0, k-1}$ and the set of the ghosts of the elements of $I_{k}$. The ghost $\varphi_{k}(x)$ for $x \in I_{k}$ is an element which is distinct from all elements of $I_{0, k-1}$. It is defined as being positioned in between the classes of the bipartition $\left(A_{x}, B_{x}\right)$ of the set $I_{0, k-1}$, endowed with the complete preorder $\succsim_{0, k-1}$, with $A_{x} \supseteq \varphi_{k-1}\left(A_{x}^{k-1}\right)$ and $B_{x} \supseteq \varphi_{k-1}\left(B_{x}^{k-1}\right)$.

There is some arbitrariness in the definition of the bipartition $\left(A_{x}, B_{x}\right)$, since there may be elements of $I_{0, k-2}$ lying in between $\varphi_{k-1}\left(A_{x}^{k-1}\right)$ and $\varphi_{k-1}\left(B_{x}^{k-1}\right)$. We can choose to define the bipartition in different ways, for instance, we may assign to $B_{x}$ all the elements of $I_{0, k-2}$ lying in between $\varphi_{k-1}\left(A_{x}^{k-1}\right)$ and $\varphi_{k-1}\left(B_{x}^{k-1}\right)$. Instead, we may assign them all to $A_{x}$.

In the rest of this section, we select one of the possible options and show that it leads to the definition of a $d$-separable complete preorder on $I_{0, k}$.

For $x \in I_{k}$, let now $J(x)$ denote the set of elements of $I_{k}$ determining the same bipartition $\left(A_{x}^{k-1}, B_{x}^{k-1}\right)$ in $I_{k-1}$ as $x$. Let $L(x)$ be the set of elements ${ }^{7}$ of $I_{0, k-1}$ which lie between $\varphi_{k-1}\left(A_{x}^{k-1}\right)$ and $\varphi_{k-1}\left(B_{x}^{k-1}\right)$, i.e., $y \in L(x) \Leftrightarrow a \succ_{0, k-1} y \succ_{0, k-1}$ $b$, for all $a \in \varphi_{k-1}\left(A_{x}^{k-1}\right)$ and $b \in \varphi_{k-1}\left(B_{x}^{k-1}\right)$. We define the bipartition $\left(A_{x}, B_{x}\right)$ as follows:

$$
\begin{aligned}
& A_{x}=L(x) \cup\left\{z \in I_{0, k-1}: \exists a \in \varphi_{k-1}\left(A_{x}^{k-1}\right) \text { such that } z \succsim_{0, k-1} a\right\}, \\
& B_{x}=I_{0, k-1} \backslash A_{x}=\left\{y \in I_{0, k-1}: \exists b \in \varphi_{k-1}\left(B_{x}^{k-1}\right) \text { such that } b \succsim_{0, k-1} y\right\} .
\end{aligned}
$$

We insert ghosts as follows. We set $a \succsim_{0, k} \varphi_{k}\left(x^{\prime}\right) \succsim_{0, k} \varphi_{k}\left(x^{\prime \prime}\right) \succ_{0, k} b$, for all $a \in A_{x}, b \in B_{x}$ and $x^{\prime}, x^{\prime \prime} \in J(x)$ with $x^{\prime} \succsim x^{\prime \prime}$. We also impose $\varphi_{k}\left(x^{\prime}\right) \succ_{0, k} \varphi_{k}\left(x^{\prime \prime}\right)$ whenever $x^{\prime} \succ x^{\prime \prime}$ (see Figure 2). In the particular case in which $A_{x}$ has a least element $\underline{a}$ (w.r.t. $\left.\succsim_{0, k-1}\right)$ and $J(x)$ has a greatest element $\bar{x}$ (w.r.t. $\succsim$ ), we set $^{8}$ $\varphi_{k}(\bar{x}) \sim_{0, k}$ a. The relation $\succsim_{0, k}$ on $I_{0, k}$ is completely defined as follows. For all $z_{1}, z_{2} \in I_{0, k}$, we have the following cases:

Case 1 if $z_{1}, z_{2} \in I_{0, k-1}$, we have $z_{1} \succsim_{0, k} z_{2}$ iff $z_{1} \succsim_{0, k-1} z_{2}$, hence $\succsim_{0, k}$ extends $\succsim_{0, k-1}$;

Case 2 if $z_{1}=\varphi_{k}\left(x_{1}\right)$ and $z_{2}=\varphi_{k}\left(x_{2}\right)$ for some $x_{1}, x_{2} \in I_{k}$, we set $z_{1} \succ_{0, k} z_{2}$ iff $x_{1} \succ x_{2}$; note that this definition is compatible with the construction above also in case $x_{1}$ and $x_{2}$ determine the same bipartition in $I_{k-1}$;

\footnotetext{
${ }^{7}$ Actually, $L(x)$ is empty in case $k=1$ and $L(x) \subseteq I_{0, k-2}$ if $k \geq 2$.

${ }^{8}$ Note that $\underline{a}$ is not necessarily the ghost of an element $y \in I_{k-1}$ such that $(\bar{x}, y)$ is a hollow. It can happen that a least element in $A_{x}$ is $\underline{a} \in L(x)$, which is the ghost of an element of $I_{l}$, for $0 \leq l<k-1$ (for more on this case, see Section 4.9).
} 
Case 3 if $z_{1}=\varphi_{k}\left(x_{1}\right)$ and $z_{2} \in I_{0, k-1}$, we have $z_{1} \succ_{0, k} \quad z_{2}$ iff $z_{2} \in B_{x_{1}}$ and $z_{2} \succsim_{0, k} z_{1}$ iff $z_{2} \in A_{x_{1}}$. Furthermore, $z_{2} \sim_{0, k} z_{1}$ iff $z_{2}$ is a least element in $A_{x_{1}}$ (w.r.t. $\left.\succsim_{0, k-1}\right)$ and $x_{1}$ is the greatest element in $J\left(x_{1}\right)$ (w.r.t. $\succsim$ ).

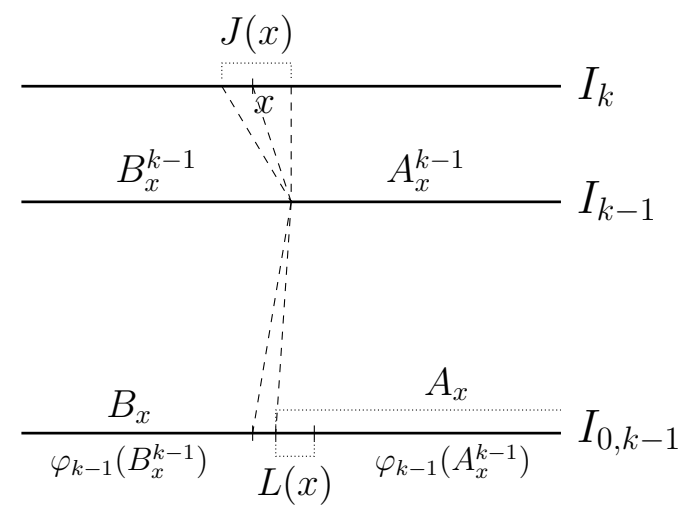

Figure 2: Ghost insertion for the elements of $I_{k}$

With this definition, we have the following result, which generalizes Lemma 14.

\section{Lemma 15}

$\succsim_{0, k}$ is a complete preorder on $I_{0, k}$ which extends $\succsim_{0, k-1}$, satisfies $\varphi_{k}(x) \succsim_{0, k} \varphi_{k}(y)$ for all $x, y \in I_{k}$ with $x \succsim y$, and is d-separable by $E_{0, k}$.

\section{ProOF}

It is easy to check that the relation $\succsim_{0, k}$ defined above is complete, transitive, extends $\succsim_{0, k-1}$. It reproduces on $\varphi_{k}\left(I_{k}\right)$ the order induced by the trace $\succsim$ on $I_{k}$.

We prove that relation $\succsim_{0, k}$ is $d$-separable. We know by Lemma 14 that $\succsim_{0,1}$ is $d$-separated by $E_{0,1}$. We prove the result by induction, assuming that $\succsim_{0, k-1}$ is $d$-separated by $E_{0, k-1}$. Let $z_{1}, z_{2} \in I_{0, k}$ be such that $z_{1} \succ_{0, k} z_{2}$.

Case 1 If $z_{1}, z_{2} \in I_{0, k-1}$ and $z_{1} \succ_{0, k} z_{2}$, we have $z_{1} \succ_{0, k-1} z_{2}$. By the induction hypothesis, we know that $z_{1}$ and $z_{2}$ can be separated by an element from $E_{0, k-1}$.

Case 2 If $z_{1}=\varphi_{k}\left(x_{1}\right)$ and $z_{2}=\varphi_{k}\left(x_{2}\right)$ for some $x_{1}, x_{2} \in I_{k}$, we know that $z_{1} \succ_{0, k} z_{2}$ implies $x_{1} \succ x_{2}$. Since $\succsim$ is $d$-separable, there is an element in $E_{k}$ separating $x_{1}$ from $x_{2}$. Therefore, $z_{1}$ and $z_{2}$ are separated by an element in $\varphi_{k}\left(E_{k}\right)$.

Case 3 If $z_{1}=\varphi_{k}\left(x_{1}\right)$ and $z_{2} \in I_{0, k-1}$, we have that $z_{1} \succ_{0, k} z_{2}$ implies that $z_{2} \in B_{x_{1}}$. If $z_{2}$ is not a greatest element in $B_{x_{1}}$ (w.r.t. $\succsim_{0, k-1}$ ), then there 
is $z \in B_{x_{1}}$ such that $z_{1} \succ_{0, k} z \succ_{0, k-1} z_{2}$. By the induction hypothesis, $z_{2}$ can be separated from $z$, and therefore from $z_{1}$, by an element from $E_{0, k-1}$. If $z_{2}$ is a greatest element in $B_{x_{1}}$ and there is $x \in J\left(x_{1}\right)$ such that $x_{1} \succ x$, then $\varphi_{k}\left(x_{1}\right) \succ_{0, k} \varphi_{k}(x) \succ_{0, k} z_{2}$ and we can use the separability of $\succsim$. Therefore, $z_{1}$ and $z_{2}$ are separated by an element in $\varphi_{k}\left(E_{k}\right)$. Finally, we have to consider the case in which $z_{2}$ is a greatest element in $B_{x_{1}}$ and $x_{1}$ is the least element in $J\left(x_{1}\right)$. In this case, there is $y \in I_{k-1}$ such that $\left(x_{1}, y\right)$ is a nose and $\varphi_{k-1}(y) \sim_{k-1} z_{2}$. Since $y$ is a nose endpoint, it belongs to $E_{k-1}$ and $\varphi_{k-1}(y) \in E_{0, k}$ separates $z_{1}$ from $z_{2}$.

Case 4 If $z_{1} \in I_{0, k-1}$ and $z_{2}=\varphi_{k}\left(x_{2}\right)$, we have that $z_{1} \succ_{0, k} z_{2}$ implies that $z_{1} \in A_{x_{2}}$. If $z_{1}$ is not the least element in $A_{x_{2}}$ (w.r.t. $\succsim_{0, k-1}$ ), then there is $z \in A_{x_{2}}$ such that $z_{1} \succ_{0, k} z \succ_{0, k-1} z_{2}$. By the induction hypothesis, $z_{1}$ can be separated from $z$, and therefore from $z_{1}$, by an element from $E_{0, k-1}$. If $z_{1}$ is a least element in $A_{x_{2}}$ and there is $x \in J\left(x_{2}\right)$ with $x \succ x_{2}$, then $z_{1} \succ_{0, k} \varphi_{k}(x) \succ_{0, k} \varphi_{k}\left(x_{2}\right)$, and we can use the separability of $\succsim$. Therefore, $z_{1}$ and $z_{2}$ are separated by an element in $\varphi_{k}\left(E_{k}\right)$. Finally, we have to consider the case in which $z_{1}$ is a least element in $A_{x_{2}}$ and $x_{2}$ is the greatest element in $J\left(x_{2}\right)$. This is incompatible with $z_{1} \succ_{0, k} z_{2}$. Indeed, we decided to place $z_{2}=\varphi_{k}\left(x_{2}\right)$ in the equivalence class of $z_{1}$. We thus have $z_{2} \sim_{k} z_{1}$, which contradicts $z_{1} \succ_{0, k} z_{2}$.

\subsection{Construction of $I_{-1, k}$}

We assume that $I_{0, k}$ has been constructed as well as the complete preorder $\succsim_{0, k}$ on it. The set $I_{-1, k}$ is the union of $I_{0, k}$ and the set of the ghosts of the elements in $I_{-1}$. The ghost $\varphi_{-1}(x)$ for $x \in I_{-1}$ is an element which is distinct from all elements in $I_{0, k}$. It is defined as being positioned in between the classes of a bipartition $\left(C_{x}, D_{x}\right)$ of the set $I_{0, k}$, endowed with the complete preorder $\succsim_{0, k}$, with $C_{x} \supseteq C_{x}^{0}$ and $D_{x} \supseteq D_{x}^{0}$. There is some arbitrariness in the definition of the bipartition $\left(C_{x}, D_{x}\right)$, since there may be elements of $I_{0, k}$ lying in between $C_{x}^{0}$ and $D_{x}^{0}$.

We select one of the possible options and show that it leads to the definition of a $d$-separable complete preorder on $I_{-1, k}$. For $x \in I_{-1}$, let now $K(x)$ denote the set of elements of $I_{-1}$ determining the same bipartition $\left(C_{x}^{0}, D_{x}^{0}\right)$ in $I_{0}$ as $x$. Let $M(x)$ be the set of elements in $I_{0, k}$ which lie between $C_{x}^{0}$ and $D_{x}^{0}$, i.e., $y \in M(x) \Leftrightarrow c \succ_{0, k} y \succ_{0, k} d$, for all $c \in C_{x}^{0}$ and $d \in D_{x}^{0}$. We define the bipartition $\left(C_{x}, D_{x}\right)$ as follows:

$$
\begin{aligned}
& C_{x}=\left\{z \in I_{0, k}: \exists c \in C_{x}^{0} \text { such that } z \succsim_{0, k} c\right\}, \\
& D_{x}=I_{0, k} \backslash C_{x}=M(x) \cup\left\{y \in I_{0, k}: \exists d \in D_{x}^{0} \text { such that } d \succsim_{0, k} y\right\} .
\end{aligned}
$$


We insert ghosts as follows. We set $c \succsim_{-1, k} \varphi_{-1}\left(x^{\prime}\right) \succsim_{-1, k} \varphi_{-1}\left(x^{\prime \prime}\right) \succsim_{-1, k} d$, for all $c \in C_{x}, d \in D_{x}$ and $x^{\prime}, x^{\prime \prime} \in K(x)$ with $x^{\prime} \succsim x^{\prime \prime}$. We also impose $\varphi_{-1}\left(x^{\prime}\right) \succ_{-1, k}$ $\varphi_{-1}\left(x^{\prime \prime}\right)$ whenever $x^{\prime} \succ x^{\prime \prime}$ (see Figure 3 ). In the particular case in which $D_{x}$ has a greatest element $\bar{d}$ (w.r.t. $\succsim_{0, k}$ ) and $K(x)$ has a least element $\underline{x}$ (w.r.t. $\succsim$ ), we $\operatorname{set}^{9} \varphi_{-1}(\underline{x}) \sim_{-1, k} \bar{d}$. The relation $\succsim_{-1, k}$ on $I_{-1, k}$ is completely defined as follows. For all $z_{1}, z_{2} \in I_{-1, k}$, we have the following cases:

Case 1 if $z_{1}, z_{2} \in I_{0, k}$, we have $z_{1} \succsim_{-1, k} z_{2}$ iff $z_{1} \succsim_{0, k} z_{2}$, hence $\succsim_{-1, k}$ extends $\succsim_{0, k}$;

Case 2 if $z_{1}=\varphi_{-1}\left(x_{1}\right)$ and $z_{2}=\varphi_{-1}\left(x_{2}\right)$ for some $x_{1}, x_{2} \in I_{-1}$, we set $z_{1} \succ_{-1, k} z_{2}$ iff $x_{1} \succ x_{2}$; note that this definition is compatible with the construction above also in case $x_{1}$ and $x_{2}$ determine the same bipartition in $I_{0}$;

Case 3 if $z_{1}=\varphi_{-1}\left(x_{1}\right)$ and $z_{2} \in I_{0, k}$, we have $z_{1} \succsim_{-1, k} z_{2}$ iff $z_{2} \in D_{x_{1}}$, and $z_{2} \succ_{-1, k} z_{1}$ iff $z_{2} \in C_{x_{1}}$. Furthermore, $z_{2} \sim_{-1, k} z_{1}$ iff $z_{2}$ is a greatest element in $D_{x_{1}}$ (w.r.t. $\left.\succsim_{0, k}\right)$ and $x_{1}$ is the least element in $K\left(x_{1}\right)$ (w.r.t. $\left.\succsim\right)$.

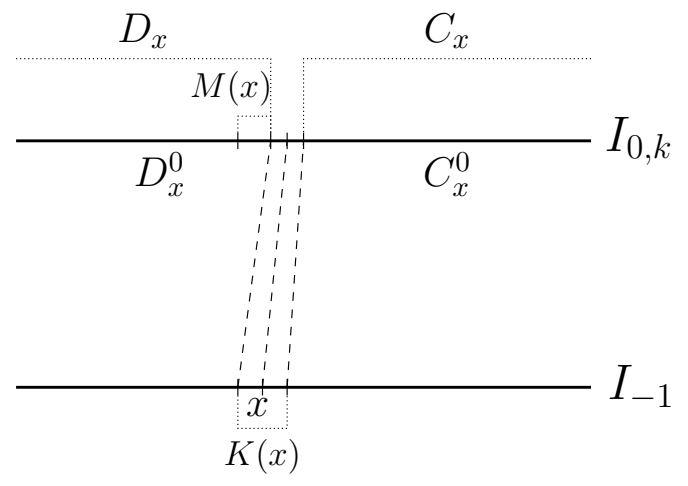

Figure 3: Ghost insertion for $I_{-1}$ into $I_{0, k}$

With this definition, the following lemma, which extends Lemma 15, holds.

\section{Lemma 16}

$\succsim_{-1, k}$ is a complete preorder on $I_{-1, k}$ which extends $\succsim_{0, k}$, satisfies $\varphi_{k}(x) \succsim_{-1, k}$ $\varphi_{k}(y)$ for all $x, y \in I_{-1}$ with $x \succsim y$, and is d-separable by $E_{-1, k}$.

\section{PROOF}

It is easy to check that the relation $\succsim_{-1, k}$ defined above is complete, transitive and extends $\succsim_{0, k}$. It reproduces on $\varphi_{k}\left(I_{-1}\right)$ the order induced by the trace $\succsim$ on $I_{-1}$. We prove that relation $\succsim_{-1, k}$ is $d$-separable. We know by Lemma 15 that $\succsim_{0, k}$ is $d$-separated by $E_{0, k}$. Let $z_{1}, z_{2} \in I_{-1, k}$ be such that $z_{1} \succ_{-1, k} z_{2}$.

\footnotetext{
${ }^{9}$ Note that $(\bar{d}, \underline{x})$ is a hollow only if $\bar{d} \in I_{0}$. Actually, $\bar{d}$ could belong to $M(x)$ and be the ghost of an element in $I_{l}$, for $1 \leq l \leq k$. In such a case $(\bar{d}, \underline{x})$ is no hollow.
} 
Case 1 If $z_{1}, z_{2} \in I_{0, k}$ and $z_{1} \succ_{-1, k} z_{2}$, we have $z_{1} \succ_{0, k} z_{2}$. By Lemma 15, we know that $z_{1}$ and $z_{2}$ can be separated by an element from $E_{0, k}$.

Case 2 If $z_{1}=\varphi_{-1}\left(x_{1}\right)$ and $z_{2}=\varphi_{-1}\left(x_{2}\right)$ for some $x_{1}, x_{2} \in I_{-1}$, we know that $z_{1} \succ_{-1, k} z_{2}$ implies $x_{1} \succ x_{2}$. Since $\succsim$ is $d$-separable, there is an element in $E_{-1}$ separating $x_{1}$ from $x_{2}$. Therefore, $z_{1}$ and $z_{2}$ are separated by an element in $\varphi_{-1}\left(E_{-1}\right)$.

Case 3 If $z_{1}=\varphi_{-1}\left(x_{1}\right)$ and $z_{2} \in I_{0, k}$, we have that $z_{1} \succ_{-1, k} z_{2}$ implies that $z_{2} \in D_{x_{1}}$. If $z_{2}$ is not a greatest element in $D_{x_{1}}$ (w.r.t. $\succsim_{0, k}$ ), then there is $z \in D_{x_{1}}$ such that $z_{1} \succ_{-1, k} z \succ_{0, k} z_{2}$. By Lemma $15, z_{2}$ can be separated from $z$, and therefore from $z_{1}$, by an element from $E_{0, k}$. If $z_{2}$ is a greatest element in $D_{x_{1}}$ and there is $x \in M\left(x_{1}\right)$ such that $x_{1} \succ x$, then $\varphi_{-1}\left(x_{1}\right) \succ_{-1, k}$ $\varphi_{-1}(x) \succ_{-1, k} z_{2}$, and we can use the separability of $\succsim$. Therefore, $z_{1}$ and $z_{2}$ are separated by an element in $\varphi_{-1}\left(E_{-1}\right)$. Finally, we have to consider the case in which $z_{2}$ is a greatest element in $D_{x_{1}}$ and $x_{1}$ is the least element in $K\left(x_{1}\right)$. This is incompatible with $z_{1} \succ_{-1, k} z_{2}$ because, in this case, we have decided that $z_{1}=\varphi_{-1}\left(x_{1}\right) \sim_{-1, k} z_{2}$.

Case 4 If $z_{1} \in I_{0, k}$ and $z_{2}=\varphi_{-1}\left(x_{2}\right)$, we have that $z_{1} \succ_{0, k} z_{2}$ implies $z_{1} \in C_{x_{2}}$. If $z_{1}$ is not a least element in $C_{x_{2}}$ (w.r.t. $\succsim_{0, k}$ ), then there is $z \in C_{x_{2}}$ such that $z_{1} \succ_{-1, k} z \succ_{0, k} z_{2}$. By Lemma $15, z_{1}$ can be separated from $z$, and therefore from $z_{2}$, by an element from $E_{0, k}$. If $z_{1}$ is a least element in $C_{x_{2}}$ and there is $x \in I_{-1}$ with $z_{2} \succ_{0, k} \varphi_{-1}(x) \succ_{0, k} \varphi_{-1}\left(x_{2}\right)$, then we can use the separability of $\succsim$. Therefore, $z_{1}$ and $z_{2}$ are separated by an element in $\varphi_{-1}\left(E_{-1}\right)$. Finally, we have to consider the case in which $z_{1}$ is a least element in $C_{x_{2}}$ and $x_{2}$ is the greatest element in $K\left(x_{2}\right)$. In this case, there is $y \in I_{0}$ such that $\left(y, x_{2}\right)$ is a nose and $y \sim_{0, k} z_{1}$. Since $y$ is a nose endpoint, it belongs to $E_{0}$ and it separates $z_{1}$ from $z_{2}$.

\subsection{Construction of $I_{-l, k}$}

This is the general step of the construction. Actually, depending on the order in which we perform the ghosts insertions, we may need to build $I_{-l, k}$ starting either from $I_{-l+1, k}$ or from $I_{-l, k-1}$. We describe the construction of $I_{-l, k}$ starting from $I_{-l+1, k}$, leaving the construction starting from $I_{-l, k-1}$ to the reader.

We assume that $I_{-l+1, k}$ has been constructed as well as the complete preorder $\succsim_{-l+1, k}$ on it. The set $I_{-l, k}$ is the union of $I_{-l+1, k}$ and the set of the ghosts of the elements in $I_{-l}$. The ghost $\varphi_{-l}(x)$ for $x \in I_{-l}$ is an element which is distinct from all elements of $I_{-l+1, k}$. It is defined as being positioned in between the classes of a bipartition $\left(C_{x}, D_{x}\right)$ of the set $I_{-l+1, k}$, endowed with the complete preorder $\succsim_{-l+1, k}$, 
with $C_{x} \supseteq \varphi_{-l+1}\left(C_{x}^{-l+1}\right)$ and $D_{x} \supseteq \varphi_{-l+1}\left(D_{x}^{-l+1}\right)$. There is some arbitrariness in the definition of the bipartition $\left(C_{x}, D_{x}\right)$, since there may be elements of $I_{-l+1, k}$ lying in between $\varphi_{-l+1}\left(C_{x}^{-l+1}\right)$ and $\varphi_{-l+1}\left(D_{x}^{-l+1}\right)$.

We select one of the possible options and show that it leads to the definition of a $d$-separable complete preorder on $I_{-l, k}$. For $x \in I_{-l}$, let $K(x)$ denote the set of elements of $I_{-l}$ determining the same bipartition $\left(C_{x}^{-l+1}, D_{x}^{-l+1}\right)$ in $I_{-l+1}$ as $x$. Let $M(x)$ be the set of elements of $I_{-l+1, k}$ which lie between $\varphi_{-l+1}\left(C_{x}^{-l+1}\right)$ and $\varphi_{-l+1}\left(D_{x}^{-l+1}\right)$, i.e., $y \in M(x) \Leftrightarrow c \succ_{0, k} y \succ_{0, k} d$, for all $c \in \varphi_{-l+1}\left(C_{x}^{-l+1}\right)$ and $d \in \varphi_{-l+1}\left(D_{x}^{-l+1}\right)$. We define the bipartition $\left(C_{x}, D_{x}\right)$ as follows:

$C_{x}=\left\{z \in I_{-l+1, k}: \exists c \in \varphi_{-l+1}\left(C_{x}^{-l+1}\right)\right.$ such that $\left.z \succsim_{-l+1, k} c\right\}$,

$D_{x}=I_{-l+1, k} \backslash C_{x}=M(x) \cup\left\{y \in I_{-l+1, k}: \exists d \in \varphi_{-l+1}\left(D_{x}^{-l+1}\right)\right.$ such that $\left.d \succsim_{-l+1, k} y\right\}$.

We insert ghosts as follows. We set $c \succsim_{-l, k} \varphi_{-l}\left(x^{\prime}\right) \succsim_{-l, k} \varphi_{-1}\left(x^{\prime \prime}\right) \succsim_{-l, k} d$, for all $c \in C_{x}, d \in D_{x}$ and $x^{\prime}, x^{\prime \prime} \in K(x)$ with $x^{\prime} \succsim x^{\prime \prime}$. We also impose $\varphi_{-l}\left(x^{\prime}\right) \succ_{-l, k}$ $\varphi_{-l}\left(x^{\prime \prime}\right)$ whenever $x^{\prime} \succ x^{\prime \prime}$ (see Figure 4 ). In the particular case in which $D_{x}$ has a greatest element $\bar{d}$ (w.r.t. $\succsim_{-l+1, k}$ ) and $K(x)$ has a least element $\underline{x}$ (w.r.t. $\succsim$ ), we $\operatorname{set}^{10} \varphi_{-l}(\underline{x}) \sim_{-l, k} \bar{d}$. The relation $\succsim_{-l, k}$ on $I_{-l, k}$ is completely defined as follows. For all $z_{1}, z_{2} \in I_{-l, k}$, we distinguish the following cases:

Case 1 if $z_{1}, z_{2} \in I_{-l+1, k}$, we have $z_{1} \succsim_{-l, k} z_{2}$ iff $z_{1} \succsim_{-l+1, k} z_{2}$, hence $\succsim_{-1, k}$ extends $\succsim-l+1, k$;

Case 2 if $z_{1}=\varphi_{-l}\left(x_{1}\right)$ and $z_{2}=\varphi_{-l}\left(x_{2}\right)$ for some $x_{1}, x_{2} \in I_{-l}$, we set $z_{1} \succ_{-l, k} z_{2}$ iff $x_{1} \succ x_{2}$; note that this definition is compatible with the construction above also in case $x_{1}$ and $x_{2}$ determine the same bipartition in $I_{-l+1}$;

Case 3 if $z_{1}=\varphi_{-l}\left(x_{1}\right)$ and $z_{2} \in I_{-l+1, k}$, we have $z_{1} \succsim_{-l, k} z_{2}$ iff $z_{2} \in D_{x_{1}}$, and $z_{2} \succ_{-l, k} z_{1}$ iff $z_{2} \in C_{x_{1}}$. Furthermore, $z_{2} \sim_{-l, k} z_{1}$ iff $z_{2}$ is a greatest element in $D_{x_{1}}$ (w.r.t. $\succsim_{-l+1, k}$ ) and $x_{1}$ is the least element in $K\left(x_{1}\right)$ (w.r.t. $\succsim$ ).

With this definition, the following lemma, which extends Lemma 16, holds.

\section{Lemma 17}

$\succsim_{-l, k}$ is a complete preorder on $I_{-l, k}$ which extends $\succsim_{-l+1, k}$, satisfies $\varphi_{k}(x) \succsim_{-l, k}$ $\varphi_{k}(y)$ for all $x, y \in I_{-l}$ with $x \succsim y$, and is d-separable by $E_{-l, k}$.

\section{ProOF}

It is easy to check that the relation $\succsim_{-l, k}$ defined above is complete, transitive and extends $\succsim_{-l+1, k}$. It reproduces on $\varphi_{k}\left(I_{-l}\right)$ the order induced by the trace $\succsim$ on $I_{-l}$.

\footnotetext{
${ }^{10}$ The pair $(\bar{d}, \underline{x})$ is a hollow if and only if $\bar{d}$ is the ghost of an element of $I_{-l+1}$. This is not always the case since $\bar{d}$ can belong to $M(x)$ (see also footnote 9 ).
} 


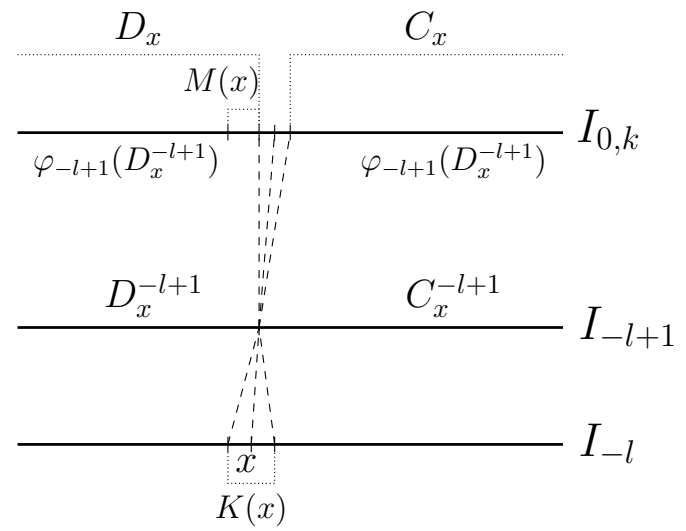

Figure 4: Ghost insertion for $I_{-l}$ into $I_{-l+1, k}$

We prove that relation $\succsim_{-l, k}$ is $d$-separable. We know by Lemma 16 that $\succsim_{-1, k}$ is $d$-separated by $E_{-1, k}$. We prove the result by induction, assuming that $\succsim_{-l+1, k}$ is $d$-separable by $E_{-l+1, k}$. Let $z_{1}, z_{2} \in I_{-l, k}$ be such that $z_{1} \succ_{-l, k} z_{2}$.

Case 1 If $z_{1}, z_{2} \in I_{-l+1, k}$ and $z_{1} \succ_{-l, k} z_{2}$, we have $z_{1} \succ_{-l+1, k} z_{2}$. By the induction hypothesis, $z_{1}$ and $z_{2}$ can be separated by an element from $E_{-l+1, k}$.

Case 2 If $z_{1}=\varphi_{-l}\left(x_{1}\right)$ and $z_{2}=\varphi_{-l}\left(x_{2}\right)$ for some $x_{1}, x_{2} \in I_{-l}$, we know that $z_{1} \succ_{-l, k} z_{2}$ implies $x_{1} \succ x_{2}$. Since $\succsim$ is $d$-separable, there is an element in $E_{-l}$ separating $x_{1}$ from $x_{2}$. Therefore, $z_{1}$ and $z_{2}$ are separated by an element in $\varphi_{-l}\left(E_{-l}\right)$.

Case 3 If $z_{1}=\varphi_{-l}\left(x_{1}\right)$ and $z_{2} \in I_{-l+1, k}$, we have that $z_{1} \succ_{-l, k} z_{2}$ implies that $z_{2} \in D_{x_{1}}$. If $z_{2}$ is not a greatest element in $D_{x_{1}}$ (w.r.t. $\succsim_{-l+1, k}$ ), then there is $z \in D_{x_{1}}$ such that $z_{1} \succ_{-l, k} z \succ_{-l+1, k} z_{2}$. By Lemma $15, z_{2}$ can be separated from $z$, and therefore from $z_{1}$, by an element from $E_{-l+1, k}$. If $z_{2}$ is a greatest element in $D_{x_{1}}$ and there is $x \in M\left(x_{1}\right)$ such that $x_{1} \succ x$, then $\varphi_{-l}\left(x_{1}\right) \succ_{-l, k} \varphi_{-1}(x) \succ_{-l, k} z_{2}$, and we can use the separability of $\succsim$. Therefore, $z_{1}$ and $z_{2}$ are separated by an element in $\varphi_{-l}\left(E_{-l}\right)$. Finally, we have to consider the case in which $z_{2}$ is a greatest element in $D_{x_{1}}$ and $x_{1}$ is the least element in $K\left(x_{1}\right)$. This is incompatible with $z_{1} \succ_{-l, k} z_{2}$ because, in this case, we decided that $z_{1}=\varphi_{-l}\left(x_{1}\right) \sim_{-l, k} z_{2}$.

Case 4 If $z_{1} \in I_{-l+1, k}$ and $z_{2}=\varphi_{-l}\left(x_{2}\right)$ for some $x_{2} \in I_{-l}$, we have that $z_{1} \succ_{-l, k} z_{2}$ implies $z_{1} \in C_{x_{2}}$. If $z_{1}$ is not a least element in $C_{x_{2}}$ (w.r.t. $\succsim-l+1, k$ ), then there is $z \in C_{x_{2}}$ such that $z_{1} \succ_{-l+1, k} z \succ_{-l, k} z_{2}$. By the induction hypothesis, $z_{1}$ can be separated from $z$, and therefore from $z_{2}$, by an element from $E_{-l+1, k}$. If $z_{1}$ is a least element in $C_{x_{2}}$ and there is $x \in K\left(x_{2}\right)$ with $x \succ x_{2}$, 
then $z_{2} \succ_{-l, k} \varphi_{-l}(x) \succ_{-l, k} \varphi_{-l}\left(x_{2}\right)$, and we can use the separability of $\succsim$. Therefore, $z_{1}$ and $z_{2}$ are separated by an element in $\varphi_{-l}\left(E_{-l}\right)$. Finally, we have to consider the case in which $z_{1}$ is a least element in $C_{x_{2}}$ and $x_{2}$ is the greatest element in $K\left(x_{2}\right)$. In this case, there is $y \in I_{0}$ such that $\left(y, x_{2}\right)$ is a nose and $\varphi_{-l+1}(y) \sim_{-l+1, k} z_{1}$. Since $y$ is a nose endpoint, it belongs to $E_{-l+1}$ and $\varphi_{-l+1}(y) \in E_{-l+1, k}$ separates $z_{1}$ from $z_{2}$.

At the end of this construction process, involving at most a countably infinite number of steps, we obtain the set

$$
\tilde{I}_{0}=I_{0} \cup\left(\bigcup_{k \geq 0} \varphi_{k}\left(I_{k}\right)\right) \cup\left(\bigcup_{l>0} \varphi_{-l}\left(I_{-l}\right)\right)=I_{0} \cup\left(\bigcup_{m \in M} \varphi_{m}\left(I_{m}\right)\right),
$$

ordered by $\succsim_{\varphi}$, which is an extension of the linear order $\succsim$ on $I_{0}$. It also extends the complete preorder $\succsim_{-l, k}$, for all $k,-l \in M$. The restriction of $\succsim_{\varphi}$ to $\varphi_{m}\left(I_{m}\right)$, for $m \in M, m \neq 0$, is an isomorphic image of the linear order $\succsim$ on $I_{k}$. The denumerable set $\tilde{E}_{0}=E_{0} \cup\left(\bigcup_{m \in M} \varphi_{m}\left(E_{m}\right)\right) d$-separates the complete preorder $\succsim_{\varphi}$. Indeed, for all $x \neq y \in \tilde{I}_{0}, x$ and $y$ belong to $I_{-l, k}$ for some $-l, k \in M$ and by Lemma 17 , they are separated by some element in $E_{-l, k}$. Note that, while $\succsim$ is a linear order on $X$ (provided no pair of distinct elements are equivalent in the semiorder, an hypothesis that was made w.l.o.g. in Section 2.1), $\succsim_{\varphi}$ is a complete preorder, in general. The equivalence class of an element may not be reduced to a singleton since there are cases in which we set a ghost equivalent to another element or another ghost in the course of the construction of $\left(\tilde{I}_{0}, \succsim_{\varphi}\right)$ (see also Remark 34 in Bouyssou and Pirlot (2020a) for other possible cases of equivalence of ghosts corresponding to elements in consecutive sets $I_{m}$ and $I_{m+1}$ ).

\section{Remark 18}

Starting from $I_{0,1}$ we may construct the ghosts of all the elements of a connected semiorder. In case the decomposition in subsets $\left(\ldots, I_{-l}, \ldots, I_{0}, \ldots, I_{k}, \ldots\right)$ involves finitely many subsets, the construction may proceed by first exhausting all positive indices $k$, i.e., by constructing $I_{0, \bar{k}}$ (using section 4.4) and then building $I_{-l, \bar{k}}$, for $l=1, \ldots, \bar{l}$ (using sections 4.5 and 4.6). In case there are a countably infinite number of subsets in the decomposition, one may proceed by addressing alternatively the positive and the negative labels. For instance: $I_{0,1}, I_{-1,1}, I_{-1,2}, I_{-2,2}, \ldots$. The previous sections allow to do that provided we consider as established the option left to the reader in section 4.6, i.e., building $I_{-l, k}$ from $I_{-l, k-1}$. 


\subsection{Construction of a representation on an $I$-connected component}

Since the complete preorder $\succsim_{\varphi}$ on $\tilde{I}_{0}$ is $d$-separable, there exists a numerical representation $f: \tilde{I}_{0} \rightarrow \mathbb{R}$ of $\succsim_{\varphi}$ such that $f(x) \geq f(y)$, for all $x, y \in \tilde{I}_{0}$ such that $x \succsim_{\varphi} y$.

In order to build a unit representation of the semiorder $S=(P, I)$, we select a numerical representation $f$ of $\succsim_{\varphi}$ into the $] 0,1[$ real interval and we define the function $u$ on $\mathcal{D}$ as follows:

$$
u(x)=f\left(\varphi_{m}(x)\right)+m \text { for all } x \in I_{m},
$$

for all $m \in M$, and interpreting $\varphi_{0}$ as the identity function.

\section{Proposition 19}

If $f: X \rightarrow] 0,1\left[\subset \mathbb{R}\right.$ is a numerical representation of $\succsim_{\varphi}$ on $\tilde{I}_{0}$, then the function $u$ defined by (6) is a unit representation of the semiorder $S=(P, I)$ restricted to $\mathcal{D}$, i.e., for all $x, y \in \mathcal{D}$,

$$
\begin{array}{cll}
u(x)>u(y)+1 & \text { iff } & x P y \\
-1 \leq u(x)-u(y) \leq 1 & \text { iff } & x I y
\end{array}
$$

\section{PROOF}

Let $x, y$ be such that $x P y$. If $y$ belongs to $I_{k}(k \in M)$, we have that $x$ belongs to $I_{m}$ for $m \geq k+1$ (by Proposition 13, items 13.4, 13.5 and 13.6). If $x \in I_{k+1}$, we have $u(x)-u(y)=f\left(\varphi_{k+1}(x)\right)+k+1-f\left(\varphi_{k}(y)\right)-k>1$, since $\varphi_{k+1}(x) \succ_{\varphi} \varphi_{k}(y)$ by construction, and therefore $f\left(\varphi_{k+1}(x)\right)>f\left(\varphi_{k}(y)\right)$.

If $x \in I_{m}$, for $m \geq k+2$, we have $u(x)-u(y)=f\left(\varphi_{m}(x)\right)+m-f\left(\varphi_{k}(y)\right)-k \geq 1$ since $m-k \geq 2$ and $\left|f\left(\varphi_{m}(x)\right)-f\left(\varphi_{k}(y)\right)\right| \leq 1$. Actually, $u(x)-u(y)>1$. Assume for contradiction that $u(x)-u(y)=1$. This implies that $m=k+2$ and $f\left(\varphi_{m}(x)\right)-f\left(\varphi_{k}(y)\right)=-1$. The latter means that $\varphi_{m}(x)$ is the least element in $\left(\tilde{I}_{0}, \succsim_{\varphi}\right)$ and $\varphi_{k}(y)$ is the largest. Consider first the case in which $m>0$. Since $\varphi_{m}(x)$ is the least element in $\tilde{I}_{0}$, we have that $\varphi_{m}(x) \precsim \varphi \varphi_{m-1}(z)$, for all $z \in I_{m-1}$. Therefore, $x I z$, for all $z \in I_{m-1}$. This implies that $x \in I_{m-1}$, by construction of $I_{m-1}$, a contradiction. Turning to the case in which $m \leq 0$, i.e., $k \leq-2$, we deduce similarly that $y I w$, for all $w \in I_{k+1}$. This implies that $y \in I_{k+1}$, a contradiction.

Consider now a pair $x, y \in \mathcal{D}$ such that $x I y$. We assume w.l.o.g. that $x \succ y$ and $y \in I_{k}(k \in M)$. By Proposition 13, items 13.2, 13.3 and 13.5, we know that $x \in I_{k}$ or $x \in I_{k+1}$. In the former case, $0<u(x)-u(y)=f\left(\varphi_{k}(x)\right)+k-f\left(\varphi_{k}(y)\right)-k \leq 1$, since $0 \leq f \leq 1$. In the latter case, we have $0<u(x)-u(y)=f\left(\varphi_{k+1}(x)\right)+k+$ $1-f\left(\varphi_{k}(y)\right)-k<1$ because $f\left(\varphi_{k+1}(x)\right)-f\left(\varphi_{k}(y)\right) \leq 0$. To establish this, we consider the following two possible cases: 
- $k \geq 0$. By construction of the ghosts and the extension $\succsim_{\varphi}$ of $\succsim$ for $k \geq 0$, we have $\varphi_{k}(a) \succsim_{\varphi} \varphi_{k+1}(x) \succ_{\varphi} \varphi_{k}(b)$ for all $a \in A=\left\{z \in I_{k}: x I z\right\}$ and all $b \in B=\left\{z \in I_{k}: x P z\right\}$. Since $y$ belongs to $A$ and $f$ represents $\succsim_{\varphi}$, we have that $f\left(\varphi_{k}(y)\right)>f\left(\varphi_{k+1}(x)\right)$.

- $k=-l<0$. By construction of the ghosts and the extension $\succsim_{\varphi}$ of $\succsim$ for $k=-l<0$, we have $\varphi_{-l+1}(c) \succ_{\varphi} \varphi_{-l}(y) \succsim_{\varphi} \varphi_{-l+1}(d)$ for all $c \in C=\{z \in$ $\left.I_{-l+1}: z P y\right\}$ and all $d \in D=\left\{z \in I_{-l+1}: z I y\right\}$. Since $x$ belongs to $D$ and $f$ represents $\succsim_{\varphi}$, we have that $f\left(\varphi_{-l}(y)\right)=f\left(\varphi_{k}(y)\right) \geq f\left(\varphi_{-l+1}(x)\right)=$ $f\left(\varphi_{k+1}(x)\right)$.

\section{Remark 20}

Choosing a representation $f$ ranging in the ]0,1[ interval is restrictive, as observed in Bouyssou and Pirlot (2020a), in the subsection of Section 4.2 devoted to the construction of a representation. In contrast with the latter paper, we do not intend here to guarantee that all possible numerical representations of the semiorder can be obtained by our construction. Such an opportunity was already lost in the procedure we followed for inserting the ghosts, which is described in Sections 4.4, 4.5 and 4.6. We indeed selected a particular way of inserting the new ghosts in the previously constructed extension of $I_{0}$. Therefore, in the sequel we just aim at showing the existence of a representation.

The next result gives necessary and sufficient conditions for an $I$-connected semiorder to have a strict unit representation. It is thus a generalization of Manders (1981, Prop. 8, p. 237) and Bouyssou and Pirlot (2020a, Prop. 36), which deal with denumerable semiorders. Note that the Bounded $P$-chain condition is implied by I-connectedness (see Bouyssou and Pirlot, 2020a, Prop. 54). Hence it need not be imposed here.

\section{Proposition 21}

Let $S=(P, I)$ be an I-connected semiorder on the set $X$ and let $\succsim$ be its trace. $S$ admits a unit strict representation $u$ satisfying (1) iff the complete preorder $\succsim$ is $d$-separable and $S$ has an at most denumerable set of noses.

\section{ProOF}

Both conditions are necessary. If $u$ is a numerical representation of $S$ and $x \succ y$ then $u(x)>u(y)$. We may impose that $u$ assigns the same value to all the elements in the same equivalence class of $\sim$. In such a case $u$ is a numerical representation of the complete preorder $\succsim$. It is well-known that the existence of a denumerable set $d$-separating $\succsim$ is a necessary and sufficient condition for the existence of a numerical representation of this complete preorder (Krantz et al., 
1971, Th. 2, p. 40). We showed in Remark 11 that no representation satisfying (1) exists when the set of noses is uncountable.

The construction of $\tilde{I}_{0}$ endowed with the complete preorder $\succsim_{\varphi}$ and Proposition 19 establish the existence of a numerical representation of $S$ satisfying (1).

\subsection{Assembling representations on $I$-connected components}

We now consider the general case in which the semiorder $S=(P, I)$ on $X$ is not necessarily $I$-connected. The strategy followed in the denumerable case can also be used in the general case (Bouyssou and Pirlot, 2020a, Remark 64). Assuming that a strict unit representation exists for the restrictions of the semiorder to each of its $I$-connected components, we assemble them into a strict unit representation of the whole semiorder. In order to do so, we need assuming that the semiorder satisfies the Bounded $P$-chain condition. The next proposition is our main result concerning strict unit representations. It is a reformulation of Candeal and Induráin (2010, Th. 3.6, p. 487) in which the $s$-separability condition is factorized into $d$-separability of the trace and denumerability of the set of noses. We emphasize that this factorization refines both the $s$-separability condition and the $P$-gap-edge-points condition of Beja and Gilboa (1992) (see Remark 12 above).

\section{Theorem 22}

Let $S=(P, I)$ be a semiorder on the set $X$ and let $\succsim$ be its trace. $S$ admits a unit numerical representation $u$ satisfying (1) iff the semiorder $S$ satisfies the Bounded $P$-chain condition, the complete preorder $\succsim i s ~ d-s e p a r a b l e ~ a n d ~ S$ has an at most denumerable set of noses. The latter three conditions are independent.

\section{ProOF}

The necessity of the Bounded P-chain condition (Property 1 ) is obvious. If there were an infinite $P$-chain in a interval $[a, b]$ (i.e., the set $\{x: a \precsim x \precsim b\}$ ), it would not be possible to assign a finite value to both $u(a)$ and $u(b)$. A numerical representation $u$ of the semiorder satisfies $u(a)>u(b)$ whenever $a \succ b$. A representation of the semiorder might not be a representation of the trace $\succsim$ in the sense that it might distinguish some equivalent elements, i.e., one might have that $u(a)>u(b)$ for some $a \sim b$. In such a case one may always transform $u$ in a representation $u^{\prime}$ of the semiorder that gives the same value to all elements of each equivalence class of $\succsim$. As a result $u^{\prime}$ is a numerical representation of $\succsim$, which cannot exist if $\succsim$ is not $d$-separable (Krantz et al., 1971, Th. 2, p. 40). It was shown in Remark 11 that a representable semiorder has an at most denumerable set of noses.

We now show the sufficiency of the conditions. Assuming Property 1, Proposition 23 in Bouyssou and Pirlot (2020a) implies that a representation of a semiorder can be built whenever there is a representation of the restrictions of the semiorder 
to each of its $I$-connected components. If the whole semiorder has an at most denumerable set of nodes, it is also the case of its restrictions to all $I$-connected components. If the trace $\succsim$ is $d$-separable so are its restrictions to all $I$-connected components. Therefore, applying Proposition 21, we know that there exists a numerical representation on each I-connected component. Using Remark 64 in Bouyssou and Pirlot (2020a), we know how to assemble them into a representation of the whole semiorder.

To prove the independence of the three conditions, consider the following three examples.

\section{Example 23}

Let $X=\mathbb{R}^{2}$. Consider the binary relation $S$ such that $S=P \cup I$ with $\left(x_{1}, x_{2}\right) P$ $\left(y_{1}, y_{2}\right)$ if $x_{1}>y_{1}+1$ or $\left[x_{1}=y_{1}+1\right.$ and $\left.x_{2}>y_{2}\right]$, while $I$ is the symmetric complement of $P$ (i.e., $x I y \Leftrightarrow \operatorname{Not}[x P y]$ and $\operatorname{Not}\left[\begin{array}{ll}y & P\end{array}\right]$ ).

It is not difficult to show that $S$ is a semiorder (that is not a complete preorder). It is clear that for all $x, y \in X$, there is an $I$-chain joining them, so that this semiorder is $I$-connected and therefore the Bounded $P$-chain condition holds. The set of noses of $S$ is easily seen to be empty. The trace of $S$ is the lexicographic preorder on $\mathbb{R}^{2}$. Hence, $d$-separability is violated (see Beardon et al., 2002, Bridges and Mehta, 1995).

\section{Example 24}

Let $S=(P, I)$ be the semiorder on $\mathbb{R}$ defined by $x P y \Leftrightarrow x \geq y+1$, while $I$ is the symmetric complement of $P$. For all $x, y \in \mathbb{R}$, there is a $P$-chain joining them, so that the Bounded $P$-chain condition holds. The trace $\succsim$ of $S$ is $\geq$ on $\mathbb{R}$, so that the trace is $d$-separable. All ordered pairs $(x, y) \in \mathbb{R}^{2}$ such that $x=y+1$ are noses, which violates the denumerable noses condition.

\section{Example 25}

Let $X=\mathbb{N} \cup\{\omega\}$. Consider the binary relation $S$ such that $\omega P x$, for all $x \in \mathbb{N}$ and $x P y$ iff $x>y+1$, for all $x, y \in \mathbb{N}$, while $I$ is the symmetric complement of $P$. Since $X$ is denumerable, $d$-separability and the condition on noses trivially hold. The Bounded P-chain condition is violated.

\subsection{Hollows-faithful representations}

In this section (which the reader may wish to skip without prejudice for the understanding of the rest of the paper) we investigate the existence of a special type of strict representation that we shall call hollows-faithful representations. The latter is a strict unit representation in which the values associated to the endpoints of 
each hollow differ by exactly one unit. In the construction of the set $\tilde{I}_{0}$ and the complete preorder $\succsim_{\varphi}$ detailed in Sections 4.3 to 4.6, we placed, in some particular cases, the ghost of an element in $I_{k}, k>1$ (resp. in $I_{-l}, l>1$ ) into the equivalence class (w.r.t. $\succsim_{\varphi}$ ) of the ghost of an element in $I_{k-1}$ (resp. in $I_{-l+1}$ ). In the construction of $I_{0,1}$ (see Section 4.3), the ghost $\varphi_{1}(x)$ of an element $x$ in $I_{1}$ is set equivalent to an element $a \in I_{0}$ if and only if $(x, a)$ is a hollow (see also footnote 6 , page 13). When applying (6) to a numerical representation of the complete preorder $\succsim_{\varphi}$ on $\tilde{I}_{0}$, we obtain a strict representation $u$, with $u(x)=u(a)+1$ for all hollows $(x, a), x \in I_{1}, a \in I_{0}$.

However, in all the other steps of the construction of $\left(\tilde{I}_{0}, \succsim_{\varphi}\right)$, there is no guarantee that ghosts put in the same equivalence class always correspond to hollows. This is emphasized in footnotes 8,9 and 10 .

We shall prove below that a semiorder admits a hollows-faithful representation as soon as the semiorder has a strict unit representation. The proof will imply to slightly modify the ghost insertion procedure (Sections 4.4 to 4.6). It also requires to bring some change in the partitions $\left(I_{m}, m \in M\right)$ to avoid that both endpoints of a hollow might belong to the same class $I_{m}$.

\section{Proposition 26 (Existence of hollows-faithful representations)}

Let $S=(P, I)$ be a semiorder on the set $X$ and let $\succsim$ be its trace. If the semiorder $S$ satisfies the Bounded $P$-chain condition, the complete preorder $\succsim$ is d-separable and $S$ has an at most denumerable set of noses, then $S$ admits a unit strict numerical representation u satisfying:

$$
u(x)-u(y)=1 \quad \text { iff } \quad(x, y) \text { is a hollow. }
$$

\section{ProOF}

For proving the result, it suffices to establish the existence of a special strict unit representation satisfying (7) for each $I$-connected component $\mathcal{D}$ of $(X, S)$. Indeed, assembling representations that fulfill (7) as described in Section 4.8 preserves this property. We thus consider any $I$-connected component $\mathcal{D}$ of the semiorder. There are two problems with the current construction of a strict unit representation that need to be solved in view of constructing a representation satisfying (7): (i) both hollow endpoints belonging to consecutive classes of a partition $\left(I_{m}, m \in M\right)$ should be assigned to the same equivalence class of the complete preorder $\succsim_{\varphi}$; (ii) hollow endpoints should not belong to the same class of the partition. We first show how to solve (i) by slightly modifying the ghost insertion procedure described in Sections 4.3 to 4.6. Then we show how to modify a partition $\left(I_{m}, m \in M\right)$ in order to solve (ii) by avoiding the presence of both endpoints of a hollow in the same class.

(i) Hollows-faithful insertion procedure. 
$I_{0,1}:$ no modification.

$I_{0, k}$ : only the insertion of the ghosts of elements $x \in I_{k}$ such that $(x, y)$ is a hollow for some $y \in I_{k-1}$. In such a case, $x$ is the largest element in $J(x)$ and $\varphi_{k-1}\left(A_{x}^{k-1}\right)$ has a least element $\varphi_{k-1}(y)$. Instead of inserting $\varphi_{k}(x)$ between $A_{x}$ and $B_{x}$ as described in Section 4.4, we set $\varphi_{k}(x) \sim_{0, k} \varphi_{k-1}(y)$. This means that we insert $\varphi_{k}(x)$ in between $A_{x} \backslash L(x)$ and $B_{x} \cup L(x)$, setting it indifferent to a least element in $A(x) \backslash L(x)$. With this modified insertion, Lemma 15 remains true. Its proof need only be adapted in Cases 3 and 4 . Regarding Case 3, nothing changes unless $x_{1}$ is the endpoint of a hollow $\left(x_{1}, y\right)$ with $y \in I_{k-1}$. In such a case, we have $\varphi_{k}\left(x_{1}\right) \sim_{0, k} \varphi_{k-1}(y)$. If $z_{1}=\varphi_{k}\left(x_{1}\right)$ and $z_{2} \in I_{0, k-1}$, we have that $z_{1} \succ_{0, k} z_{2}$ implies that $z_{2} \in B_{x_{1}} \cup L\left(x_{1}\right)$. By the induction hypothesis, there is $z \in E_{0, k-1}$ which separates $\varphi_{k-1}(y)$ and $z_{2}$, i.e., $\varphi_{k-1}(y) \succsim_{0, k-1} z \succsim_{0, k-1} z_{2}$. Since $z_{1} \sim_{0, k} \varphi_{k-1}(y)$ and $\succsim_{0, k}$ extends $\succsim_{0, k-1}$, we have that $z \in E_{0, k-1}$ also separates $z_{1}$ and $z_{2}$ in $\left(I_{0, k}, \succsim_{0, k}\right)$. A similar argument applies in Case 4.

$I_{-1, k}$ : only the insertion of the ghosts of elements $x \in I_{-1}$ such that $(y, x)$ is a hollow for some $y \in I_{0}$. In such a case, $x$ is the least element in $J(x)$ and $\varphi_{-1}\left(D_{x}^{-1}\right)$ has a largest element $y$. Instead of inserting $\varphi_{-1}(x)$ between $C_{x}$ and $D_{x}$ as described in Section 4.5, we set $\varphi_{-1}(x) \sim_{-1, k} y$. This means that we insert $\varphi_{-1}(x)$ in between $D_{x} \backslash M(x)$ and $C_{x} \cup M(x)$, setting it indifferent to a largest element in $D(x) \backslash M(x)$. With this modified insertion, Lemma 16 remains true. Its proof need only be adapted in Cases 3 and 4. Regarding Case 3, nothing changes unless $x_{1}$ is the endpoint of a hollow $\left(y, x_{1}\right)$ with $y \in I_{0}$. In such a case, we have $\varphi_{-1}\left(x_{1}\right) \sim_{-1, k} y$. If $z_{1}=\varphi_{-1}\left(x_{1}\right)$ and $z_{2} \in I_{0, k}$, we have that $z_{1} \succ_{-1, k} z_{2}$ implies that $z_{2} \in D_{x_{1}} \cup M\left(x_{1}\right)$. By the induction hypothesis, there is $z \in E_{0, k}$ which separates $y$ and $z_{2}$, i.e., $y \succsim_{0, k} z \succsim_{0, k} z_{2}$. Since $z_{1} \sim_{-1, k} y$ and $\succsim_{-1, k}$ extends $\succsim_{0, k}$, we have that $z \in E_{0, k}$ also separates $z_{1}$ and $z_{2}$ in $\left(I_{-1, k}, \succsim_{-1, k}\right)$. A similar argument applies in Case 4.

$I_{-l, k}$ : only the insertion of the ghosts of elements $x \in I_{-l}$ such that $(y, x)$ is a hollow for some $y \in I_{-l+1}$. In such a case, $x$ is the least element in $J(x)$ and $\varphi_{-1}\left(D_{x}^{-l+1}\right)$ has a largest element $\varphi_{-l+1}(y)$. Instead of inserting $\varphi_{-l}(x)$ between $C_{x}$ and $D_{x}$ as described in Section 4.6, we set $\varphi_{-l}(x) \sim_{-l, k} \varphi_{-l+1}(y)$. This means that we insert $\varphi_{-l}(x)$ in between $D_{x} \backslash M(x)$ and $C_{x} \cup M(x)$, setting it indifferent to a largest element in $D(x) \backslash M(x)$. With this modified insertion, Lemma 17 remains true. Its proof need only be adapted in Cases 3 and 4. Regarding Case 3, nothing changes unless $x_{1}$ is the endpoint of a hollow $\left(y, x_{1}\right)$ with $y \in I_{-l+1}$. In such a case, we have $\varphi_{-l}\left(x_{1}\right) \sim_{-l, k} \varphi_{-l+1} y$. If $z_{1}=\varphi_{-l}\left(x_{1}\right)$ and $z_{2} \in I_{-l+1, k}$, we have that $z_{1} \succ_{-l, k} z_{2}$ implies that $z_{2} \in D_{x_{1}} \cup M\left(x_{1}\right)$. By the induction hypothesis, there is $z \in E_{-l+1, k}$ which 
separates $\varphi_{-l+1}(y)$ and $z_{2}$, i.e., $\varphi_{-l+1}(y) \succsim_{-l+1, k} z \succsim_{-l+1, k} z_{2}$. Since $z_{1} \sim_{-l, k} y$ and $\succsim_{-l, k}$ extends $\succsim_{-l+1, k}$, we have that $z \in E_{-l+1, k}$ also separates $z_{1}$ and $z_{2}$ in $\left(I_{-l, k}, \succsim_{-l, k}\right)$. A similar argument applies in Case 4.

Applying the usual construction of a representation described in Section 4.7, we obtain a strict unit representation of the semiorder restricted to the $I$-connected component $\mathcal{D}$. Such a representation fulfills $(7)$ for all hollows $(x, y)$ for which $x$ and $y$ belong to different indifference classes $I_{m}$ and $I_{m+1}$.

(ii) Modifying the partition $\left(I_{m}, m \in M\right)$.

With the construction of the partition $\left(I_{m}, m \in M\right)$ described in Bouyssou and Pirlot (2020a, Section 3), it may happen that both endpoints of a hollow belong to the same indifference class $I_{m}$ (see Example 27 below). In order to obtain a partition $\left(I_{m}^{\prime}, m \in M^{\prime}\right)$ without hollows inside a class $I_{m}^{\prime}$, for all $m \in M^{\prime}$, we may restructure the initial partition as follows.

- Search for the smallest $k \geq 0$ such that $I_{k}$ contains both endpoints $x, y$ of a hollow $(x, y)$. If this is the case for $I_{k}$, let $I_{k}^{\prime}=I_{k} \backslash\{x\}$. Transfer $x$ to $I_{k+1}^{\prime}$ and, define $I_{k+1}^{\prime}$ as the maximal indifference class whose least element is $x$; however, if this class has a greatest element $z$, and $(z, x)$ is a hollow, then transfer $z$ to $I_{k+2}$. Continue in this way, adapting the construction principles explained in Bouyssou and Pirlot (2020a, Section 3) in order to avoid the existence of hollows with both endpoints in the same class. If no $I_{k}, k \geq 0$ containing both endpoints of a hollow is found, keep the classes $\left(I_{k}, k \geq 0\right)$ unchanged.

- Search for the smallest $l>0$ such that $I_{-l}$ contains both endpoints $x, y$ of a hollow $(x, y)$. If this is the case for $I_{-l}$, let $I_{-l}^{\prime}=I_{-l} \backslash\{y\}$. Transfer $y$ to $I_{-l-1}^{\prime}$ and, define $I_{-l-1}^{\prime}$ as the maximal indifference class whose largest element is $y$; however, if this class has a least element $w$, and $(y, w)$ is a hollow, then transfer $w$ to $I_{-l-2}$. Continue in this way, adapting the construction principles explained in Bouyssou and Pirlot (2020a, Section 3) in order to avoid the existence of hollows with both endpoints in the same class. If no $I_{-l}, l>0$ containing both endpoints of a hollow is found, keep the classes $\left(I_{-l}, l \geq 0\right)$ unchanged.

The obtained partition $\left(I_{m}^{\prime}, m \in M^{\prime}\right)$ has no class which contains both endpoints of a hollow. Applying the modified ghost insertion procedure described in the beginning of this proof to the partition $\left(I_{m}^{\prime}, m \in M^{\prime}\right)$ yields a strict unit representation that fulfills condition (7).

We illustrate the previous result and its proof in the following example. 


\section{Example 27}

Consider the semiorder $S=(P, I)$ defined on a set $X$ as follows. Let $X$ be the following union of real intervals: $X=[-0.5,1.9[\cup[2.1,2.9] \cup] 3.1,5.1]$ endowed with the restriction to $X$ of the usual strict unit semiorder on $\mathbb{R}$. Assume that the partition $I_{m}, m=-1,0, \ldots 5$ is the one represented in Figure 5 .

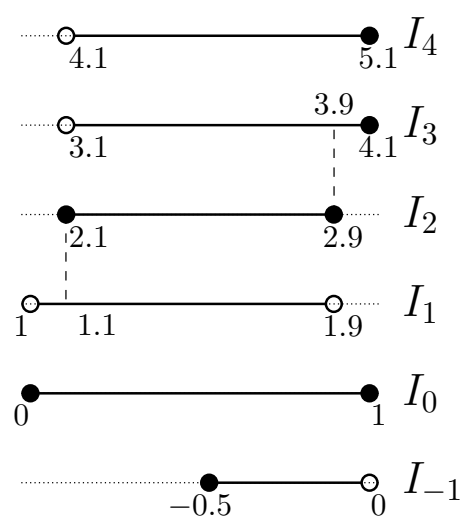

Figure 5: Partition in indifference classes for the semiorder $(X, S)$. An empty (resp. filled) circle indicates that the segment is open (resp. closed).

The pair $(2.9,2.1)$ in $I_{2}$ is a hollow. In the numerical representation that defines the semiorder, the difference between the values associated to the endpoints of this hollow is equal to 0.8. The question we want to solve is whether there is a strict unit representation of this semiorder in which the endpoints values of all hollows differ by exactly one unit. Below, we modify the partition $\left(I_{m}, m=-1, \ldots, 4\right)$ in order to avoid having hollow endpoints in the same class (see Figure 6). Then we illustrate the application of the modified ghost insertion procedure described in the proof of Proposition 26.

Let us start with $I_{0}$. This class contains the hollow $(1,0)$. We move 1 to $I_{1}^{\prime}$. $I_{1}^{\prime}=\left[1,1.9\left[\right.\right.$. In $I_{2},(2.9,2.1)$ is a hollow. We move 2.9 to $I_{3}^{\prime}$, leaving $I_{2}^{\prime}=[2.1,2.9[$. $I_{3}^{\prime}$ starts with 2.9 up to 3.9. We do not include 3.9 in $I_{3}^{\prime}$ in order to avoid including the hollow $(3.9,2.9)$ in a class. So $I_{3}^{\prime}=\{2.9\} \cup\left[3.1,3.9\left[. I_{4}^{\prime}=[3.9,4.9[\right.\right.$. Finally, $I_{5}^{\prime}=[4.9,5.1]$. There is one more class than in the original partition.

The modified ghost insertion procedure, applied to $\left(I_{m}^{\prime}, m=-1, \ldots, 5\right)$, yields $\tilde{I}_{0}=I_{-1,5}$ ordered by $\succsim_{\varphi}$, as represented in Table 1 . We start with the insertion of the ghosts of the elements of $I_{1}^{\prime}$ into $I_{0}^{\prime}$. All elements of $I_{1}^{\prime}$ are the upper endpoint of a hollow, the other endpoint being in $1_{0}^{\prime}$. Therefore, for all $x \in I_{1}^{\prime}=[1,1.9[$, its ghost $\varphi_{1}(x)$ is equivalent to the other endpoint $y$ of the hollow $(x, y)$, i.e., $\varphi_{1}(x) \sim_{0,1} y=x-1$. This is shown in the rows labeled $I_{0}^{\prime}$ and $I_{1}^{\prime}$ in Table 1. Important elements of $I_{0}^{\prime}$ have been singled out, namely, $0,0.1,0.5$ and 0.9 , because 


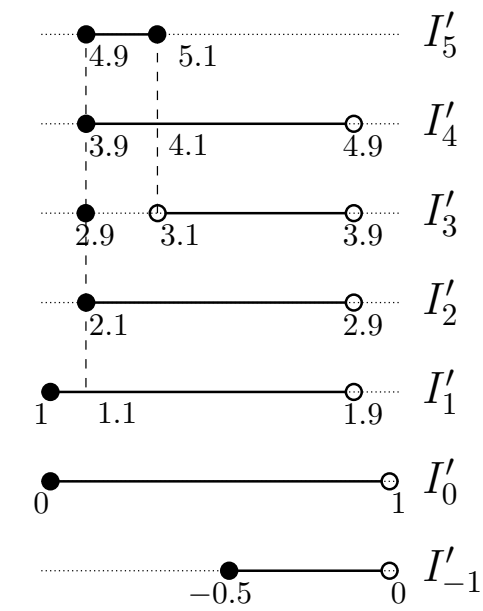

Figure 6: Modified partition $\left(I_{m}^{\prime}, m=-1, \ldots, 5\right)$ for the semiorder $(X, S)$

they correspond to transitions in $\tilde{I}_{0}(0.3$ does not correspond to a particular element in $I_{0}^{\prime}$; it is used to create a gap in which some ghosts from $I_{4}^{\prime}$ and $I_{5}^{\prime}$ need to be inserted). We see that the endpoints of each hollow are inserted in the same column of the table (single hollow endpoints in the columns headed by singular values; intervals coupled in the hollow relation in intervals between singular values). Each element in $I_{2}^{\prime}=\left[2.1,2.9\left[\right.\right.$ is associated to an element of $\left[1.1,1.9\left[\subset I_{1}^{\prime}\right.\right.$ by the hollow relation. Therefore, we have, for all $x \in I_{2}^{\prime}, \varphi_{2}(x) \sim_{0,2} \varphi_{1}(x-1) \sim_{0,2} x-2$. The row labelled $I_{2}^{\prime}$ in Table 1 shows that the elements in $I_{2}^{\prime}$ are assigned to the same columns as their associate elements through the hollow relation. The insertion of the ghosts of $I_{3}^{\prime}$ is similarly constrained by the hollow relation. The least element 2.9 is associated to 2.1 because they form a hollow. Therefore the ghosts of $2.9,2.1,1.1$ and 0.1 are indifferent w.r.t. $\sim_{0,3}$. The elements of ]3.1,3.9[ are coupled to those of ]2.1,2.9[. This is shown in Table 1 where the elements of ] $3.1,3.9$ [ are assigned to the columns between 0.3 and 0.9 just as the corresponding elements of $] 2.1,2.9\left[\right.$. It is the insertion of $I_{4}^{\prime}$ that creates the gap in the table. The least element 3.9 is coupled to 2.9 and placed in the column headed by 0.1 . The elements in $] 3.9,4.1$ ] are not upper endpoints of a hollow. Their ghosts have to be inserted between those of 2.9 and those of all the elements of the interval ]3.1,3.9[. Therefore, they are positioned in the table in the column between 0.1 and 0.3 and 4.1 is assigned to the column headed (arbitrarily) by 0.3 . The elements $I_{5}^{\prime}$ are coupled to these in $[3.9,4.1] \subset I_{4}^{\prime}$. Their position in the table results from this constraint. Finally, since the elements of $I_{-1}^{\prime}=[-0.5,0[$ are coupled to the elements in $\left[0.5,1\left[\subset I_{0}^{\prime}\right.\right.$ by the hollow relation, forcing the position indicated in the table. 
We are now in position to explain the headings row in the table. The row label $f$ refers to a representation of the complete preorder $\succsim_{-1,5}=\succsim_{\varphi}$ on $\tilde{I}_{0}$. If we assign the heading value to the ghosts of the elements in the columns having a heading and if we linearly interpolate between these values to assign values to the ghosts in the intervals between the headed columns, we obtain a representation of $\succsim_{\varphi}$. From this, we build a representation of the semiorder, which fulfills condition (7), by using definition (6).

\begin{tabular}{|l|l|l|l|l|l|l|l|l|l|l|l|}
\hline$f$ & 0 & & 0.1 & & 0.3 & & 0.5 & & 0.9 & & 1 \\
\hline$I_{0}^{\prime}$ & 0 & ] $0,0.1[$ & 0.1 & & & ] $0.1,0.5[$ & 0.5 & ] $0.5,0.9[$ & 0.9 & ] $0.9,1[$ & \\
$I_{1}^{\prime}$ & 1 & ] $1,1.1[$ & 1.1 & & & ] $1.1,1.5[$ & 1.5 & ] $1.5,1.9[$ & & & \\
$I_{2}^{\prime}$ & & & 2.1 & & & ] $2.1,2.5[$ & 2.5 & ] $2.5,2.9[$ & & & \\
$I_{3}^{\prime}$ & & & 2.9 & & & ] $3.1,3.5[$ & 3.5 & ] $3.5,3.9[$ & & & \\
$I_{4}^{\prime}$ & & & 3.9 & ] $3.9,4.1[$ & 4.1 & ] $4.1,4.5[$ & 4.5 & ] $4.5,4.9[$ & & & \\
$I_{5}^{\prime}$ & & & 4.9 & ] $4.9,5.1[$ & 5.1 & & & & & & \\
$I_{-1}^{\prime}$ & & & & & & & -.5 & ]$-.5,-.1[$ & -.1 & ]$-.1,0[$ & \\
\hline
\end{tabular}

Table 1: $\tilde{I}_{0}$ and a function $f$ that represents the complete preorder $\succsim_{\varphi}$ on $\tilde{I}_{0}$

Figure 7 represents in an obvious manner the semiorder as well as the hollowsfaithful strict unit representation obtained by applying (6) to the numerical representation $f$ of the complete preorder $\succsim_{\varphi}$ on $\tilde{I}_{0}$.

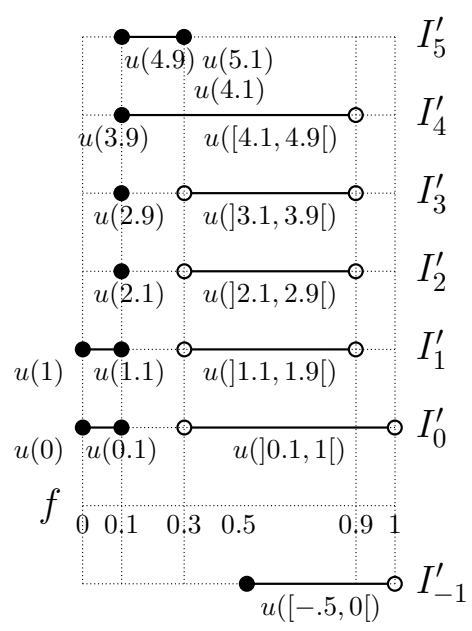

Figure 7: Hollows-faithful strict unit representation of the semiorder $(X, S)$

\section{Remark 28}

The result in Proposition 26, i.e., the existence of hollows-faithful representations as soon as strict unit representations exist, is not limited to uncountable 
semiorders. Of course it holds - and so does the proof - for denumerable semiorders under the Bounded P-chain condition, which guarantees the existence of a strict unit representation (Bouyssou and Pirlot, 2020a, Th. 64). We did not mention this result in Bouyssou and Pirlot (2020a) because our goal there was mainly to study the "uniqueness" of the representation. Therefore we did not focus on special representations but tried to show how to construct all possible representations.

\subsection{Summary of results}

In Section 4:

- We gave interpretable conditions for the existence of a strict unit representation of a semiorder in the general case. Our denumerable set of noses condition refines both the $s$-separability condition of Candeal and Induráin (2010) and the P-gap-edge-points of Beja and Gilboa (1992). The relationship between the latter two conditions is thus clarified.

- We showed that the technique for constructing a strict unit representation, that we developed in Bouyssou and Pirlot (2020a) in the denumerable case, can be adapted to deal with the general case. In addition, we showed that such a construction gives control on the representation. This assertion is illustrated by establishing the existence of special representations, namely the hollows-faithful representations.

The benefit of giving a particular role to noses and hollows will be further emphasized in the next section, which studies the existence of nonstrict unit representations. We shall see that, for nonstrict unit representations, the hollows play the role played by the noses in the case of strict unit representations.

\section{$5 \quad$ Nonstrict unit representations}

Conditions guaranteeing that a semiorder has a nonstrict unit representation, i.e., a representation satisfying (2), have been established by Beja and Gilboa (1992, Th. 4.5.b, p. 439). Candeal and Induráin (2010) did not study nonstrict unit representations but a condition "dual" to their $s$-separability condition can be formulated. The latter will play the role of $s$-separability for nonstrict unit representations. We shall write down such a condition and factorize it into $d$-separability of the trace and another condition, in the spirit of what we did for strict unit representations. The latter condition refines both the condition dual to $s$-separability and the condition of denumerability of the set of $I$-upper-edge-points used by Beja and Gilboa (1992) in their characterization. 
In the case $X$ is finite, all semiorders have both strict and nonstrict representations (see, e.g., Pirlot and Vincke (1997), Section 4.2). In case $X$ is countably infinite, every semiorder that admits a strict unit representation has also a nonstrict unit representation, and conversely (Beja and Gilboa (1992, Th. 3.8); this has already been observed by Roberts (1979, footnote, p. 36)). It is even true (Bouyssou and Pirlot, 2020a, Th. 64) that a semiorder on a denumerable set that admits a unit representation also has a unit representation $u$ that is simultaneously strict and nonstrict (i.e., $|u(x)-u(y)| \neq 1$, for all $x, y$ ). The Bounded $P$-chain condition is a necessary and sufficient condition for a semiorder on a denumerable set to have both strict and nonstrict unit representations. For a semiorder on an uncountable set it is no longer the case that it has a strict unit representation if and only if it has a nonstrict one. Consider for instance the nonstrict unit semiorder on the reals (Example 24). It has a trivial nonstrict unit representation. The set of its noses is not denumerable. Therefore, by Theorem 22, this semiorder has no strict unit representation. So, the two types of representation are not equivalent when $X$ is not denumerable.

Our goal in this section is to establish a new characterization of the semiorders that admit a nonstrict unit representation.

\subsection{Separability for nonstrict unit representations}

We introduce another form of separability that can be viewed as "dual" w.r.t. $s$ separability (Candeal and Induráin, 2010) and is useful to characterize semiorders that admit a nonstrict unit representation.

\section{Definition 29 ( $s^{\prime}$-separability)}

A semiorder $S=(P, I)$ is $s^{\prime}$-separable if there is a denumerable set $F, F \subseteq X$, such that, for all $a, b \in X$ with $a S b$, there are

$$
\begin{array}{rll}
c \in F & \text { such that } & a S c \succsim b \\
\text { and } d \in F & \text { such that } & a \succsim d S b .
\end{array}
$$

This notion of separability implies that the number of hollows (see Definition 5) of the semiorder is at most denumerable. The following lemma is the counterpart of Lemma 7 in the context of $s^{\prime}$-separability.

\section{Lemma 30}

If the semiorder $S=(P, I)$ on $X$ is $s^{\prime}$-separable by the denumerable set $F$, then a and $b$ belong to $F$ whenever $(a, b)$ is a hollow.

\section{PROOF}

Let $(a, b)$ be a hollow, thus satisfying $b I a$ hence $b S a$. By the $s^{\prime}$-separability property, there is $c \in F$ such that $b S c \succsim a$. By definition of a hollow, for all $c \succ a$, we 
have $c P b$. Therefore, $c=a$ and $a \in F$. Using $s^{\prime}$-separability, there is also $d \in F$ such that $b \succsim d S a$, which implies $b=d$ and $b \in F$.

Before proving a factorization result similar to Proposition 10, we introduce the notion of half-hollow, adapting the notion of half-noses introduced in Definition 8.

\section{Definition 31 (Half-hollows)}

The ordered pair $(a, b) \in X \times X$ is a lower half-hollow (l-h-hollow) of $S=(P, I)$ if $a I b, a \succ b$, and there is no $d \in X$ such that $b \succ d I a .(a, b)$ is a proper l-h-hollow if it is a l-h-hollow and there is $c \in X$ such that $b I c \succ a .(a, b) \in X \times X$ is an upper half-hollow (u-h-hollow) of $S=(P, I)$ if $a I b, a \succ b$, and there is no $c \in X$ such that $b I c \succ a$. $(a, b)$ is a proper $u$-h-hollow if it is an $u$-h-hollow and there is $d \in X$ such that $b \succ d I a$.

Let LHH (resp. UHH) denote the set of right endpoints b (resp. left enpoints a) of all proper l-h-hollows (resp. u-h-hollow) $(a, b)$.

The notion of upper half-hollow is closely related to that of I-upper-edge point (Beja and Gilboa, 1992, Definition P6 (b), p.438). The element $a \in X$ is an $I$-upper-edge-point if and only if there is $b \in X$ such that $(a, b)$ is an upper halfhollow. We have the following result. It is the counterpart of Lemma 9 and its proof is similar. We give it for the reader's convenience.

\section{Lemma 32}

If the semiorder $S=(P, I)$ is d-separable, then the sets $L H H$ and $U H H$ are denumerable.

\section{Proof}

We only prove the result for $L H H$. The other case is dealt with similarly.

Let $(a, b)$ be a proper l-h-hollow. We define the set $H(b)=\{x \in X: x I b, x \succ$ $b$ and $\forall d \prec b, N o t[x I d]\}$. In other words, for all $x \in H(b),(x, b)$ is a l-h-hollow. It is clear that $a \in H(b)$. Moreover, since $(a, b)$ is proper, $H(b)$ contains at least one other element $c$ such that $b I c \succ a$. $H(b)$ is an interval w.r.t. $\succ$. To show this let $x, x^{\prime} \in H(b)$. If $x^{\prime \prime}$ is such that $x \succ x^{\prime \prime} \succ x^{\prime}$, then $x^{\prime \prime} \in H(b)$. Indeed, $x^{\prime \prime} I b$ since $x I b$ and for all $d \prec b, \operatorname{Not}\left[x^{\prime \prime} I d\right]$ since $\operatorname{Not}\left[x^{\prime} I c\right]$.

Let $(a, b)$ and $\left(a^{\prime}, b^{\prime}\right)$ be two proper l-h-hollows, with $b \neq b^{\prime} \in L H H$. It is clear that the associated intervals $H(b)$ and $H\left(b^{\prime}\right)$ are disjoint. Each of these intervals contains at least two distinct points and therefore at least an element from the denumerable set $D$ that $d$-separates $S=(P, I)$. Consequently, $L H H$ is denumerable.

\section{Proposition 33}

A semiorder $S=(P, I)$ on $X$ is $s^{\prime}$-separable iff $\succsim$ is $d$-separable and the set of hollows is denumerable. 


\section{PROOF}

Assume that the semiorder is $s^{\prime}$-separable. By Lemma 30, the set of hollows is denumerable. The $s^{\prime}$-separability property implies that $\succsim$ is $d$-separable. Let $x, y \in X$ be such that $x \succ y$. There is $z \in X$ such that $x P z$ and $z S y$ and/or $w \in X$ such that $w P y$ and $x S w$. In the former case, $s^{\prime}$-separability entails that there is $c \in F$ such that $z S c \succsim y$ and, since $x P z$, we have $x \succ c \succsim y$. In the latter case, there is $d \in F$ such that $x \succsim d S w$ and, since $w P y$, we have $x \succsim d \succ y$.

Reciprocally, let $D$ be a denumerable set that $d$-separates $\succsim$. Let $x, y \in X$ be such that $y S x$. We first deal with the case $y \succ x$. By $d$-separability, there is $z \in D$ such that $x \precsim z \precsim y$, which implies $y S z$ and $z S x$. Therefore we have $y S z \succsim x$ and $y \succsim z S x$. The other case is when $x \succ y$. If $(x, y)$ is a hollow, the $s^{\prime}$-separability condition is trivially satisfied. Assuming that $(x, y)$ is not a hollow, we have that

1. either there is $x^{\prime} \succ x$ such that $y S x^{\prime}$

2. or there is $y^{\prime} \prec y$ such that $y^{\prime} S x$.

In Case 1, by the $d$-separability of $\succ$, there is $c \in D$ such that $x^{\prime} \succsim c \succsim x$. Therefore we have $y S c \succsim x$. Further, there are two cases. Either there is $y^{\prime} \prec y$ such that $y^{\prime} S x$ or for all $y^{\prime} \prec y$, we have $\operatorname{Not}\left[y^{\prime} S x\right]$. In the former case, $d$-separability implies that there is $d \in D$ such that $y^{\prime} \precsim d \precsim y$. Then, we have $y \succsim d S x$. Otherwise, $(x, y)$ is a proper l-h-hollow. In order to have $d \in F$ such that $y \succsim d S x$, we set $d=y$ and include the denumerable set $L H H$ of right endpoints of the proper l-h-hollows in $F$.

In Case 2, by the $d$-separability of $\succ$, there is $d \in D$ such that $y^{\prime} \precsim d \precsim y$. Therefore we have $x S c \precsim y$. Further, either there is $x^{\prime} \succ x$ such that $y S x^{\prime}$ or for all $x^{\prime} \succ x$, we have $\operatorname{Not}\left[y S x^{\prime}\right]$. In the former case, $d$-separability implies that there is $c \in D$ such that $x^{\prime} \succsim c \succsim x$. Then, we have $y S c \succsim x$. Otherwise, $(x, y)$ is a proper u-h-hollow. In order to have $c \in F$ such that $y S c \succsim x$, we set $c=x$ and include the denumerable set $U H H$ of left endpoints of the proper u-h-hollows in $F$.

Finally, by considering $F$ as the union of $D, L H H, U H H$ and the set of elements $a, b$ such that $(a, b)$ is a hollow, which is denumerable by hypothesis, we obtain a denumerable set $F$, which $s^{\prime}$-separates the semiorder $(P, I)$.

\section{Remark 34}

It is easy to show that having a denumerable set of hollows is a necessary condition for a semiorder to have a nonstrict unit representation. Indeed, assume that $u$ is a nonstrict unit representation of the semiorder $S=(P, I)$ and $(a, b)$ is a hollow of $S$. Since $b S a$, we have $u(b)>u(a)-1$. Let $\varepsilon_{a b}$ be the positive number $u(b)-u(a)+1$. By definition of a hollow, there is no element $c \in X$ such that $b \succ c S a$ and therefore, there is no $c$ such that $u(c) \in] u(a)-1, u(b)]$, an interval of length 
$\varepsilon_{a b}>0$. To each hollow $(a, b)$ is associated such an interval of positive length and all these intervals are disjoint. Since sets of disjoint intervals of positive length in $\mathbb{R}$ have a finite or denumerable number of elements, the number of hollows is denumerable.

\section{Remark 35}

For proving the existence of a nonstrict unit representation, we shall use $d$-separability and the condition that the number of hollows is denumerable. We could do it under the $s^{\prime}$-separability assumption, but, in the proof, we shall only use the denumerable set $D$ that is dense in the trace $\succsim$ and the denumerable set of hollows endpoints. We do not need to add the half-noses or the half-hollows as we had to do in the second part of the proof of Proposition 33. In other words, we do not use all the points in the set $F$ involved in the $s^{\prime}$-separability property (see Definition 29). In the same vein, we do not need to impose that the set of all I-upper-edge-points (Beja and Gilboa, 1992, p. 438) is denumerable. Only the cardinality of the set of $I$-upper-edge-points that correspond to hollows needs to be controlled. Our condition that the set of hollows has to be denumerable refines both the $s^{\prime}$-separability condition and the denumerability of the set of $I$-upperedge-points (Beja and Gilboa, 1992, Theorem 4.5 (b), p. 439). Its interpretation is straightforward and it clarifies the relationship between $s^{\prime}$-separability and the conditions imposed by Beja and Gilboa (1992).

\subsection{Characterization of the semiorders admitting a non- strict unit representation}

The insertion process leading to prove the existence of a strict unit representation of a semiorder can be slightly modified to accomodate nonstrict unit representations. In nonstrict representations the hollows play the role of the noses in strict representations and vice versa. We shall prove that there is a nonstrict representation of a semiorder if and only if the semiorder satisfies the Bounded $P$-chain condition, its trace is $d$-separable and the semiorder has a denumerable set of hollows.

As in Section 4, we start by proving that these conditions are sufficient. We first assume that the semiorder $S=(P, I)$ on $X$ has been decomposed into $I$-connected components. Let $\mathcal{D}$ be such a connected component. Assuming that the restriction to $\mathcal{D}$ of the semiorder has a denumerable set of hollows and that its trace is $d$ separable, we adapt the construction of $\left(\tilde{I}_{0}, \succsim_{\varphi}\right)$ described in Sections 4.4 to 4.6 in order to prove that the restriction to $\mathcal{D}$ of the semiorder admits a nonstrict unit representation (as in Section 4.7). Let $E=E^{\prime} \cup H$ be the denumerable set that is the union of all hollows endpoints $H$ and a denumerable subset $E^{\prime}$ of $X$ which 
$d$-separates the trace $\succsim$. Using the notation defined in Section 4.2 , we describe the changes in the construction of $I_{-l, k}$, for all $-l, k \in M$ with $l>0, k \geq 0$, below.

Construction of $I_{0,1}$ Ghost insertion goes as described in Section 4.3. The only difference relates to the positioning of the ghosts of the noses left endpoints (instead of the hollows left endpoints). Whenever $\left(x_{1}, b\right)$ is a nose, with $x_{1} \in I_{1}$ and $b \in I_{0}$, we place the ghost $\varphi_{1}\left(x_{1}\right)$ of $x_{1}$ in the equivalence class of $b$ in the relation $\succsim_{0,1}$. Note that, for a hollow $\left(x_{1}, a\right)$, the ghost $\varphi_{1}\left(x_{1}\right)$ is positioned strictly in between the classes of the bipartition $\left(A_{x_{1}}^{0}, B_{x_{1}}^{0}\right)$, i.e., in such a way that we have $a \succ_{0,1} \varphi_{1}\left(x_{1}\right) \succ_{0,1} b$, for all $b \in B_{x_{1}}^{0}$. There is no change in the proof that the relation $\succsim_{0,1}$ that is obtained is a complete preorder on $I_{0,1}$ extending the trace $\succsim$ on $I_{0}$ and reproducing on $\varphi_{1}\left(I_{1}\right)$ the trace order on $I_{1}$. Slight adaptation is needed to show that this complete preorder is $d$-separated by $E_{0,1}$. These occur in Cases 3 and 4 in the proof of Lemma 14. Let $z_{1} \succ_{0,1} z_{2}$ with $z_{1}, z_{2} \in I_{0,1}$.

Case 3 We consider the case $z_{1}=\varphi_{1}\left(x_{1}\right)$ and $z_{2} \in I_{0}$. We have that $z_{1} \succ_{0,1}$ $z_{2}$ implies $z_{2} \in B_{x_{1}}^{0}$. The only subcase that differs from Lemma 14 is when $x_{1}, z_{2}$ is a nose, i.e., when $z_{2}$ is the greatest element in $B_{x_{1}}^{0}$ and $x_{1}$ is the least element in $J\left(x_{1}\right)$. Actually, such a case does not occur since we have chosen to put $z_{1}=\varphi_{1}\left(x_{1}\right)$ in the equivalence class of $z_{2}$, i.e., to set $\varphi_{1}\left(x_{1}\right) \sim_{0,1} z_{2}$.

Case 4 This case deals with $z_{1} \in I_{0}$ and $z_{2}=\varphi_{1}\left(x_{2}\right)$. We have that $z_{1} \succ_{0,1}$ $z_{2}$ implies that $z_{1} \in A_{x_{2}}^{0}$. The only subcase that differs from Lemma 14 is when $z_{1}$ is the least element in $A_{x_{2}}^{0}$ and $x_{2}$ is the greatest element such that $z_{1} \succsim_{0,1} \varphi_{1}\left(x_{2}\right)$. This means that $\left(x_{2}, z_{1}\right)$ is a hollow. Since $x_{2}$ is a hollow endpoint, it belongs to $E_{0}, z_{2}=\varphi_{1}\left(x_{2}\right)$ belongs to $E_{0,1}$ and it separates $z_{1}$ from $z_{2}$.

This establishes Lemma 14 in the construction process of a nonstrict unit representation.

Construction of $I_{0, k}$ Ghosts are inserted as in Section 4.4. The bipartition $\left(A_{x}, B_{x}\right)$ of $I_{0, k-1}$ associated to element $x$ in $I_{k}$ is defined slightly differently. We choose to include in $B_{x}$ (instead of $A_{x}$ ) the set $L(x)$ of elements of $I_{0, k-2}$ which lie between $\varphi_{k-1}\left(A_{x}^{k-1}\right)$ and $\varphi_{k-1}\left(B_{x}^{k-1}\right)$. We have accordingly:

$A_{x}=\left\{y \in I_{0, k-1}: \exists a \in \varphi_{k-1}\left(A_{x}^{k-1}\right)\right.$ such that $\left.y \succsim_{0, k-1} a\right\}$,

$B_{x}=I_{0, k-1} \backslash A_{x}=L(x) \cup\left\{z \in I_{0, k-1}: \exists b \in \varphi_{k-1}\left(B_{x}^{k-1}\right)\right.$ such that $\left.b \succsim_{0, k-1} z\right\}$.

The definition of $\succsim_{0, k}$ is the same as in Section 4.4 except for Case 3, i.e., when $z_{1}=\varphi_{k}\left(x_{1}\right)$ and $z_{2} \in I_{0, k-1}$. Like in Section 4.4, we have $z_{1} \succ_{0, k} z_{2}$ 
iff $z_{2} \in A_{x_{1}}$ and $z_{2} \succsim_{0, k} z_{1}$ iff $z_{2} \in B_{x_{1}}$. The only difference is the following. When $z_{2}$ is a greatest element in $B_{x_{1}}$ (w.r.t. $\succsim_{0, k-1}$ ) and $x_{1}$ is the least element in $J\left(x_{1}\right)$ (w.r.t. $\succsim$ ), we set $z_{2} \sim_{0, k} z_{1}$.

The proof of Lemma 15 requires adaptation only in Cases 3 and 4 . Let $z_{1}, z_{2} \in I_{0, k}$ be such that $z_{1} \succ_{0, k} z_{2}$.

Case 3 If $z_{1}=\varphi_{k}\left(x_{1}\right)$ and $z_{2} \in I_{0, k-1}$, we have that $z_{1} \succ_{0, k} z_{2}$ implies that $z_{2} \in B_{x_{1}}$. The only subcase that differs from Lemma 15 is when $z_{2}$ is the greatest element in $B_{x_{1}}$ and $x_{1}$ is the least element in $J\left(x_{1}\right)$. Actually, in such a case, we chose to put $z_{1}=\varphi_{1}\left(x_{1}\right)$ in the equivalence class of $z_{2}$, i.e., to set $\varphi_{1}\left(x_{1}\right) \sim_{0,1} z_{2}$, which is not compatible with $z_{1} \succ_{0, k} z_{2}$.

Case 4 If $z_{1} \in I_{0, k-1}$ and $z_{2}=\varphi_{k}\left(x_{2}\right)$, we have that $z_{1} \succ_{0, k} z_{2}$ implies that $z_{1} \in A_{x_{2}}$. The only subcase that differs from Lemma 15 is when $z_{1}$ is a least element in $A_{x_{2}}$ and $x_{2}$ is the greatest element in $J\left(x_{2}\right)$. such that $z_{1} \succsim_{0,1} \varphi_{1}\left(x_{2}\right)$. This means that $\left(x_{2}, z_{1}\right)$ is a hollow. Since $x_{2}$ is a hollow endpoint, it belongs to $E_{k}, z_{2}=\varphi_{k}\left(x_{2}\right)$ belongs to $E_{0, k}$ and it separates $z_{1}$ from $z_{2}$.

Construction of $I_{-1, k}$ Ghosts are inserted in $I_{0, k}$ as in Section 4.5. The bipartition $\left(C_{x}, D_{x}\right)$ of $I_{0, k}$ associated to element $x$ in $I_{-1}$ is defined slightly differently. We choose to include in $C_{x}$ (instead of $D_{x}$ ) the set $M(x)$ of elements of $I_{0, k}$ which lie between $C_{x}^{0}$ and $D_{x}^{0}$, i.e., $M(x)=\left\{y \in I_{0, k}: c \succ_{0, k} y \succ_{0, k}\right.$ $\left.d, \forall c \in C_{x}^{0}, d \in D_{x}^{0}\right\}$. We have accordingly:

$$
\begin{aligned}
& C_{x}=M(x) \cup\left\{z \in I_{0, k}: \exists c \in C_{x}^{0} \text { such that } c \succsim_{0, k} z\right\}, \\
& D_{x}=I_{0, k-1} \backslash C_{x}=\left\{y \in I_{0, k}: \exists d \in D_{x}^{0} \text { such that } y \succsim_{0, k} d\right\} .
\end{aligned}
$$

The definition of $\succsim_{-1, k}$ is the same as in Section 4.4 except for Case 3, i.e., when $z_{1}=\varphi_{k}\left(x_{1}\right)$ and $z_{2} \in I_{0, k-1}$. Like in Section 4.4, we have $z_{1} \succ_{-1, k} z_{2}$ if $z_{2} \in D_{x_{1}}$, and $z_{2} \succsim_{-1, k} z_{1}$ if $z_{2} \in C_{x_{1}}$. The only difference is the following. Let $K\left(x_{1}\right)$ denote the set of elements in $I_{-1}$ determining the same bipartition $\left(C_{x_{1}}^{0}, D_{x_{1}}^{0}\right)$ as $x_{1}$. When $z_{2}$ is a least element in $C_{x_{1}}$ (w.r.t. $\succsim_{0, k}$ ) and $x_{1}$ is the greatest element in $K\left(x_{1}\right)$ (w.r.t. $\left.\succsim\right)$, we set $z_{2} \sim_{0, k} z_{1}$.

The proof of Lemma 16 requires adaptation only in Cases 3 and 4 . Let $z_{1}, z_{2} \in I_{0, k}$ be such that $z_{1} \succ_{0, k} z_{2}$.

Case 3 If $z_{1}=\varphi_{-1}\left(x_{1}\right)$ and $z_{2} \in I_{0, k}$, we have that $z_{1} \succ_{0, k} z_{2}$ implies that $z_{2} \in D_{x_{1}}$. The only subcase that differs from Lemma 16 is when $z_{2}$ is a greatest element in $D_{x_{1}}$ and $x_{1}$ is the least element in $K\left(x_{1}\right)$. By definition (9) of the bipartition, $z_{2} \in D_{x_{1}}$, hence $\left(z_{2}, x_{1}\right)$ is a hollow. Therefore $z_{2} \in E_{-1, k}$ and it trivially separates $z_{1}$ from $z_{2}$. 
Case 4 If $z_{1} \in I_{0, k}$ and $z_{2}=\varphi_{-1}\left(x_{2}\right)$, we have that $z_{1} \succ_{0, k} z_{2}$ implies that $z_{1} \in C_{x_{2}}$. The only subcase that differs from Lemma 16 is when $z_{1}$ is a least element in $C_{x_{2}}$ and $x_{2}$ is the greatest element in $K\left(x_{2}\right)$. By construction, in such a case, $z_{1} \sim_{-1, k} z_{2}=\varphi_{-1}\left(x_{2}\right)$, which contradicts $z_{1} \succ_{-1, k} z_{2}$.

Construction of $I_{-l, k}$ As in Section 4.6, we only deal with the construction of $I_{-l, k}$ starting from $I_{-l+1, k}$. Ghost insertion proceeds as in Section 4.6. The bipartition $\left(C_{x}, D_{x}\right)$ of $I_{-l+1, k}$ associated to element $x$ in $I_{-l}$ is defined slightly differently. We choose to include in $C_{x}$ (instead of $D_{x}$ ) the set $M(x)$ of elements of $I_{-l+1, k}$ which lie between $\varphi_{-l+1} C_{x}^{-l+1}$ and $\varphi_{-l+1}\left(D_{x}^{-l+1}\right)$, i.e., $M(x)=\left\{y \in I_{-l+1, k}: c \succ_{-l+1, k} y \succ_{-l+1, k} d, \forall c \in C_{x}^{-l+1}, d \in D_{x}^{-l+1}\right\}$. We have accordingly:

$$
\begin{aligned}
& C_{x}=M(x) \cup\left\{z \in I_{-l+1, k}: \exists c \in \varphi_{-l+1}\left(C_{x}^{-l+1}\right) \text { such that } c \succsim_{-l+1, k} z\right\}, \quad \text { (10) } \\
& D_{x}=I_{-l+1, k} \backslash C_{x}=\left\{y \in I_{-l+1, k}: \exists d \in \varphi_{-l+1}\left(D_{x}^{-l+1}\right) \text { such that } y \succsim_{-l+1, k} d\right\} .
\end{aligned}
$$

The definition of $\succsim_{-l+1, k}$ is the same as in Section 4.6 except for Case 3, i.e., when $z_{1}=\varphi_{-l}\left(x_{1}\right)$ and $z_{2} \in I_{-l+1, k}$. Like in Section 4.6, we have $z_{1} \succ_{-l, k} z_{2}$ iff $z_{2} \in D_{x_{1}}$ and $z_{2} \succ_{-l, k} z_{1}$ iff $z_{2} \in C_{x_{1}}$. The only difference is the following. Let $K\left(x_{1}\right)$ denote the set of elements in $I_{-l}$ determining the same bipartition $\left(C_{x_{1}}^{-l+1}, D_{x_{1}}^{-l+1}\right)$ as $x_{1}$. When $z_{2}$ is a least element in $C_{x_{1}}$ (w.r.t. $\left.\succsim_{-l+1, k}\right)$ and $x_{1}$ is the least element in $K\left(x_{1}\right)$ (w.r.t. $\left.\succsim\right)$, we set $z_{2} \sim_{-l, k} z_{1}$.

The proof of Lemma 16 requires adaptation only in Cases 3 and 4 . Let $z_{1}, z_{2} \in I_{-l, k}$ be such that $z_{1} \succ_{-l, k} z_{2}$.

Case 3 If $z_{1}=\varphi_{-l}\left(x_{1}\right)$ and $z_{2} \in I_{-l+1, k}$, we have that $z_{1} \succ_{0, k} z_{2}$ implies that $z_{2} \in D_{x_{1}}$. The only subcase that differs from Lemma 16 is when $z_{2}$ is a greatest element in $D_{x_{1}}$ and $x_{1}$ is the least element in $K\left(x_{1}\right)$. By definition (10) of the bipartition, $z_{2} \in D_{x_{1}}$, hence $\left(z_{2}, x_{1}\right)$ is a hollow. Therefore $z_{2} \in E_{-l, k}$ and it trivially separates $z_{1}$ from $z_{2}$.

Case 4 If $z_{1} \in I_{-l+1, k}$ and $z_{2}=\varphi_{-l}\left(x_{2}\right)$, we have that $z_{1} \succ_{0, k} z_{2}$ implies that $z_{1} \in C_{x_{2}}$. The only subcase that differs from Lemma 16 is when $z_{1}$ is a least element in $C_{x_{2}}$ and $x_{2}$ is the greatest element in $K\left(x_{2}\right)$. By construction, in such a case, $z_{1} \sim_{-l, k} z_{2}=\varphi_{-l}\left(x_{2}\right)$, which contradicts $z_{1} \succ_{-l, k} z_{2}$.

At the end of this construction process, involving at most a countably infinite number of steps, we obtain, abusing notation, the set $\tilde{I}_{0}=I_{0} \cup\left(\bigcup_{k \geq 0} \varphi_{k}\left(I_{k}\right)\right) \cup$ $\left(\bigcup_{l>0} \varphi_{-l}\left(I_{-l}\right)=I_{0} \cup\left(\bigcup_{m \in M} \varphi_{m}\left(I_{m}\right)\right)\right.$, ordered by $\succsim_{\varphi}$, which is an extension of the complete preorder $\succsim$ on $I_{0}$. It also extends the order $\succsim_{-l, k}$, for all $k,-l \in M$. The 
denumerable set $\tilde{E}_{0}=E_{0} \cup \bigcup_{m \in M} \varphi_{m}\left(E_{m}\right) d$-separates the complete preorder $\succsim_{\varphi}$. Indeed, for all $x \neq y \in \tilde{I}_{0}, x$ and $y$ belong to $I_{-l, k}$ for some $-l, k \in M$ and by Lemma 17, they are separated by some element in $E_{-l, k}$. The complete preorder $\succsim_{\varphi}$ thus admits a numerical representation on the real numbers.

Leaning on this construction, we prove the following proposition, which is the counterpart of Proposition 19.

\section{Proposition 36}

If $f: X \rightarrow] 0,1\left[\subset \mathbb{R}\right.$ is a numerical representation of the complete preorder $\succsim_{\varphi}$ on $\tilde{I}_{0}$, then the function $u$ defined by (6) is a nonstrict unit representation of the semiorder $S=(P, I)$ restricted to $\mathcal{D}$, i.e., for all $x, y \in \mathcal{D}$,

$$
\begin{array}{cll}
u(x) \geq u(y)+1 & \text { iff } & x P y \\
-1<u(x)-u(y)<1 & \text { iff } & x I y
\end{array}
$$

\section{ProOF}

The proof is very similar to that of Proposition 19. We give it for the reader's convenience.

Let $x, y$ be such that $x P y$. If $y$ belongs to $I_{k}(k \in M)$, we have that $x$ belongs to $I_{m}$ for $m \geq k+1$ (by Proposition 13, items 13.4, 13.5 and 13.6). If $x \in I_{k+1}$, we have $u(x)-u(y)=f\left(\varphi_{k+1}(x)\right)+k+1-f\left(\varphi_{k}(y)\right)-k \geq 1$, since $\varphi_{k+1}(x) \succsim_{\varphi} \varphi_{k}(y)$ by construction, and therefore $f\left(\varphi_{k+1}(x)\right) \geq f\left(\varphi_{k}(y)\right)$. Equality occurs when

If $x \in I_{m}$, for $m \geq k+2$, we have $u(x)-u(y)=f\left(\varphi_{m}(x)\right)+m-f\left(\varphi_{k}(y)\right)-k>1$ since $m-k \geq 2$ and $\left|f\left(\varphi_{m}(x)\right)-f\left(\varphi_{k}(y)\right)\right|<1$.

Consider now a pair $x, y \in \mathcal{D}$ such that $x I y$. We assume w.l.o.g. that $x \succ y$ and $y \in I_{k}(k \in M)$. By Proposition 13, items 13.2, 13.3 and 13.5, we know that $x \in I_{k}$ or $x \in I_{k+1}$. In the former case, $0<u(x)-u(y)=f\left(\varphi_{k}(x)\right)+k-f\left(\varphi_{k}(y)\right)-k<1$, since $0<f<1$. In the latter case, we have $0<u(x)-u(y)=f\left(\varphi_{k+1}(x)\right)+k+$ $1-f\left(\varphi_{k}(y)\right)-k<1$ because $f\left(\varphi_{k+1}(x)\right)-f\left(\varphi_{k}(y)\right)<0$. To establish this, we consider the following two possible cases:

- $k \geq 0$. By construction of the ghosts and the extension $\succsim_{\varphi}$ of $\succsim$ for $k \geq 0$, we have $\varphi_{k}(a) \succ_{\varphi} \varphi_{k+1}(x) \succ_{\varphi} \varphi_{k}(b)$ for all $a \in A=\left\{z \in I_{k}: x I z\right\}$ and all $b \in B=\left\{z \in I_{k}: x P z\right\}$. Since $y$ belongs to $A$ and $f$ represents $\succsim_{\varphi}$, we have that $f\left(\varphi_{k}(y)\right)>f\left(\varphi_{k+1}(x)\right)$.

- $k=-l<0$. By construction of the ghosts and the extension $\succsim_{\varphi}$ of $\succsim$ for $k=-l<0$, we have $\varphi_{-l+1}(c) \succ_{\varphi} \varphi_{-l}(y) \succ_{\varphi} \varphi_{-l+1}(d)$ for all $c \in C=\{z \in$ $\left.I_{-l+1}: z P y\right\}$ and all $d \in D=\left\{z \in I_{-l+1}: z I y\right\}$. Since $x$ belongs to $D$ and $f$ represents $\succsim_{\varphi}$, we have that $f\left(\varphi_{-l}(y)\right)=f\left(\varphi_{k}(y)\right)>f\left(\varphi_{-l+1}(x)\right)=$ $f\left(\varphi_{k+1}(x)\right)$. 
To save space, we do not write down explicitly the counterpart of Proposition 21 for I-connected components. It obviously holds and we leave its proof to the reader. The following result states necessary and sufficient conditions for the existence of a nonstrict unit representation of a semiorder. It is a counterpart of Theorem 22 .

\section{Theorem 37}

Let $S=(P, I)$ be a semiorder on the set $X$ and let $\succsim$ be its trace. The following conditions are equivalent.

1. S admits a nonstrict unit numerical representation u satisfying (2);

2. S satisfies the Bounded P-chain condition, the complete preorder $\succsim$ is $d$ separable and $S$ has a denumerable set of hollows;

3. S satisfies the Bounded P-chain condition and is $s^{\prime}$-separable.

The conditions listed in item 2 are independent.

\section{ProOF}

The equivalence of the second and third items results from Proposition 33. The conditions stated in item 2 are necessary for a semiorder to admit a nonstrict unit representation. The necessity of Property 1 and of the $d$-separability of the trace $\succsim$ is shown exactly as for strict representations (see the proof of Theorem 22). A semiorder that has a nonstrict unit representation cannot involve an uncountable set of hollows. This is shown in Remark 34 .

Regarding the sufficiency of these conditions, consider any $I$-connected component $\mathcal{D}$ of the semiorder. If the semiorder has a denumerable set of hollows and its trace is $d$-separable, these properties are inherited by the restrictions of the semiorder and of its trace to $\mathcal{D}$. Therefore, we may apply the construction of $\left(\tilde{I}_{0}, \succsim_{\varphi}\right)$ detailed in the beginning of the present section. Using Proposition 36, we obtain a nonstrict unit representation of the restriction of the semiorder to $\mathcal{D}$. Such a representation can be obtained for each $I$-connected component. Under the Bounded P-chain condition, we know (Bouyssou and Pirlot, 2020a, Remark 64) that it is possible to assemble the unit representations on all connected components into a unit representation of the whole semiorder.

To prove the independence of the three conditions listed in item 2, we slightly modify Examples 23, 24 and 25.

\section{Example 38}

Let $X=\mathbb{R}^{2}$. Consider the binary relation $S$ such that $S=P \cup I$ with $\left(x_{1}, x_{2}\right) P$ $\left(y_{1}, y_{2}\right)$ if $x_{1}>y_{1}+1$ or $\left[x_{1}=y_{1}+1\right.$ and $\left.x_{2} \geq y_{2}\right]$, while $I$ is the symmetric complement of $P$ (i.e., $x I y \Leftrightarrow \operatorname{Not}[x P y]$ and $\operatorname{Not}\left[\begin{array}{ll}y & P\end{array}\right]$ ). 
The only difference w.r.t. Example 23 is that the nonstrict inequality $x_{2} \geq y_{2}$ replaces the strict inequality $x_{2}>y_{2}$ in the definition of $P$. This results in an empty set of hollows, trivially implying the condition on hollows. One shows as in Example 23 that the Bounded $P$-chain condition holds and that $d$-separability is violated.

\section{Example 39}

Let $S=(P, I)$ be the semiorder on $\mathbb{R}$ defined by $x P y$ if $x>y+1$, while $I$ is the symmetric complement of $P$. This relation satisfies the Bounded $P$-chain condition. Its trace is $\geq$ on $\mathbb{R}$; it is therefore $d$-separable. Every pair $(x, y) \in \mathbb{R}^{2}$ with $x=y+1$ is a hollow, hence $S$ does not satisfy the denumerable hollows condition.

\section{Example 40}

Let $X=\mathbb{N} \cup\{\omega\}$. Consider the binary relation $S$ such that $\omega P x$, for all $x \in \mathbb{N}$ and $x P y$ iff $x \geq y+1$, for all $x, y \in \mathbb{N}$, while $I$ is the symmetric complement of $P$. Since $X$ is denumerable, $d$-separability and the condition on hollows trivially hold. The Bounded P-chain condition is violated.

\section{Remark 41}

We proved in preamble of Section 5 that a semiorder that has a nonstrict unit representation need not have a strict unit representation. We are now in a position to prove the converse. Consider for instance the semiorder in Example 39. The set of its hollows is not denumerable. Therefore, by Theorem 37, this semiorder has no nonstrict unit representation.

\section{Remark 42 (Noses-faithful representations)}

In Section 4.9 we showed that all semiorders that admit a strict unit representation also admit a special one in which the endpoints of all hollows are separated by exactly one unit in the representation (hollows-faithful representation). This result has been established by modifiying the ghost insertion procedure leading to the construction of $\left(\tilde{I}_{0}, \succsim_{\varphi}\right)$. This modification consists in placing the ghosts of the hollows in order that they are equivalent w.r.t. $\succsim_{\varphi}$. For any semiorder that admits a nonstrict representation, a similar trick can be used to construct a nonstrict representation in which the values assigned to the endpoints of each nose differ by exactly one unit. We call such a representation noses-faithful. Such a representation is obtained by modifying the construction of $\left(\tilde{I}_{0}, \succsim_{\varphi}\right)$ described in the beginning of Section 5 in the same spirit as what has been done in Section 4.9 for strict representations. For nonstrict representations, the insertion procedure should set the ghosts of noses endpoints as equivalent w.r.t. the $\succsim_{\varphi}$ relation. We leave it to the reader to convince herself that this modification is possible and can be done in a way that preserves the essential properties of $\succsim_{\varphi}$ on $\tilde{I}_{0}$. 


\subsection{Both strict and nonstrict unit representation}

A third sort of numerical representation of a semiorder can be investigated using the tools that we developed to tackle strict unit representations and nonstrict ones.

\section{Definition 43}

A strict-nonstrict unit representation of the semiorder $S=(P, I)$ on the set $X$ is a function $u$ from $X$ to $\mathbb{R}$ such that, for all $x, y \in X$,

$$
\begin{array}{cl}
u(x)>u(y)+1 & \text { if } \quad x P y \\
-1<u(x)-u(y)<1 & \text { if } \quad x I y
\end{array}
$$

With such a representation, the values of two elements of $X$ never differ by exactly one unit.

For semiorders on a denumerable set, it is well-known that they have a strict representation if and only if they have a nonstrict one (Beja and Gilboa, 1992). Moreover, if such a semiorder has a representation (strict or nonstrict), it has a strict-nonstrict one. This is no longer the case with semiorders on an uncountable set.

\section{Proposition 44}

A semiorder on an uncountable set admits a strict-nonstrict representation iff it satisfies the Bounded P-chain condition (Property 1), its trace is d-separable and the number of noses and hollows is at most denumerable.

ProOF

Necessity immediately results from Theorems 22 and 37 since a strict-nonstrict representation is both a strict unit representation and a nonstrict one.

These conditions are also sufficient. This can be proved by adapting the method used for constructing $\left(\tilde{I}_{0}, \succsim_{\varphi}\right)$ for strict unit representations and nonstrict ones. When inserting ghosts of noses or hollows endpoints, one has to make sure that the ghost of any nose (resp. hollow) endpoint and the corresponding other endpoint are never equivalent w.r.t. to the preorder $\succsim_{\varphi}$. It is easy to check that the construction described in Section 4 actually allows to do that in case noses and hollows are denumerable (see also footnote 6, p. 13).

\section{Discussion}

\subsection{Summary}

In this paper we have investigated the conditions under which a semiorder on a set of any cardinality admits a unit representation, either strict or nonstrict. 
We have exhibited a set of three independent conditions that are necessary and sufficient for the existence of a strict unit representation of a semiorder. This has been achieved by factorizing $s$-separability into $d$-separability and the condition that the set of noses is denumerable. We feel that these three conditions have a clear interpretation.

The bounded $P$-chain condition deals with the fact that the threshold is constant and positive. As noted in Bouyssou and Pirlot (2020a, Remark 50), it resembles an Archimedean condition. It applies as soon as the set $X$ is infinite, even countably infinite. It is not specific to strict unit representations. It is easy to check that it is also a necessary condition for nonstrict unit representations.

The $d$-separability condition ensures that the trace of the semiorder, which is a complete preorder, has a numerical representation. This is clearly necessary for strict unit representations but is not specific to them. As can be easily checked, $d$-separability is also necessary for nonstrict unit representations.

Our final condition states that the set of noses is denumerable. It is specific to strict unit representations. For obtaining necessary and sufficient conditions guaranteeing the existence of nonstrict unit representations, we keep unchanged the first two conditions used in the case of strict representations. The third condition is obtained by replacing our condition on "noses" by a condition on the dual notion of "hollows", in a very natural way.

Our results are linked to the discussion in Candeal and Induráin (2010, Sec. 4, p. 489) of Theorem 4.5 in Beja and Gilboa (1992, p. 439). This theorem asserts that a "Generalized Numerical Representation" (GNR) with $\mathscr{S}$ open exists iff $S$ is a semiorder (for which $\succsim$ is antisymmetric) satisfying $d$-separability and the bounded $P$-chain condition and such that the set of $P$-gap-edge-points is denumerable (see below Definition 8, for a definition of $P$-gap-edge-points).

As noted by Candeal and Induráin (2010), the proof of this result (see Beja and Gilboa, 1992, p. 446-448) refers to "positive threshold GNR in which $\mathscr{S}$ is open". This is tantamount to what we have called a strict unit representation. Hence, Candeal and Induráin (2010) wonder whether Beja and Gilboa (1992) were the first to characterize semiorders having a strict unit representation. They state (p. 489, last par. of 2nd col.) that the result in Beja and Gilboa (1992) should be amended by the addition of a condition stating, in our terms, that the set of all right endpoints of lower-half noses should be denumerable.

Our results allow us to be more specific. It is clear that if $x$ is a $P$-gap-edge-point, there is a $y$ such that $(y, x)$ is a nose or a proper lower-half nose (see Definitions 5 and 8). In other terms, $x$ is the right endpoint of a l-h-nose. A l-h-nose, is either a proper l-h-nose or a nose. Whenever $d$-separability is in force, we do not have to ensure the fact that the set of right endpoints of proper l-h-noses is denumerable (Lemma 9). We only have to require that the set of noses is denumerable, which is 
clearly implied by the requirement that the set of all right endpoints of l-h noses is denumerable: requiring that the set of $P$-gap-edge-points is denumerable therefore implies that the set of right endpoints of noses as well as the set of right endpoints of proper l-h-noses are denumerable. Proposition 10 and Theorem 22 show that this is sufficient to guarantee the existence of a strict unit representation. This condition can be weakened however since, as shown in Lemma 9, $d$-separability implies that the set of proper l-h-noses is denumerable. Hence, our result sharpens Beja and Gilboa's result discussed in Candeal and Induráin (2010, Sec. 4), while ensuring its correctness. To bring our result closer to the one of Beja and Gilboa, we could require that the set of all right endpoints of noses is denumerable instead of requiring that the set of all noses is denumerable. Clearly, these two conditions are equivalent: to the right endpoint of a nose corresponds a unique nose (see Bouyssou and Pirlot, 2020b, Remark 1).

A similar discussion can be written down for nonstrict representations. We have shown that Beja and Gilboa's condition that the set of $I$-upper-edge-points is denumerable can be weakened into our condition that the set of hollows is denumerable. Indeed, an $I$-upper-edge-point is the left endpoint of an upper half-hollow (Definition 31). The latter is either a proper upper half-hollow or a hollow. By Lemma 32, we know that the set of proper upper half hollows is denumerable as soon as the semiorder is $d$-separable. Therefore, we do not need to impose that the set of proper upper half-hollows is denumerable. For a $d$-separable semiorder, as soon as the set of hollows is denumerable, the set of upper half-hollows, hence the set of $I$-upper-edge-points, is also denumerable. The similarity with the discussion of the case of strict representations can be made complete. We formulated a condition in the spirit of Candeal and Induráin's $s$-separability condition, which is necessary for the existence of nonstrict representations. This $s^{\prime}$-separability condition is equivalent to $d$-separability of the trace and the condition that the set of hollows is denumerable. Its relationship with Beja and Gilboa's condition that the set of $I$-upper-edge-points is denumerable, can be analyzed as we did above for the case of strict representations.

For characterizing the semiorders that admit either strict or nonstrict unit representations, we used the same proof idea. This proof technique is elementary (we only rely on the existence of numerical representations for complete preorders that are $d$-separable) and constructive (at least in the case of finite semiorders, the ghost construction can be actually implemented to build numerical representations (see Bouyssou and Pirlot, 2020a, Remark 39). It is also unifying. Variants of the same proof technique allow to deal with uncountable and denumerable semiorders, and with the case of strict and nonstrict representations. It allows to prove the existence of various types of special representation: hollows-faithful (Section 4.9), noses-faithful (Remark 42) and strict-nonstrict representations (Section 5.3). We 
believe that this proof technique can be fruitful for solving other questions.

\subsection{Some directions for further work}

Let us first observe that we have left open the question of uniqueness of the representation (be it strict or nonstrict) for semiorders on uncountable sets. This is in contrast with what we did for semiorders on denumerable sets. In Bouyssou and Pirlot (2020a), we sought to keep the construction process of a unit representation as general as possible, in order to guarantee that any unit representation can be obtained by this process (see Bouyssou and Pirlot, 2020a, Prop. 46 and Remark 62). This allowed us to have a good control on the degrees of freedom in the set of unit representations of a semiorder on a denumerable set. In the uncountable case, we made an arbitrary choice of a manner of inserting ghosts that guarantees the $d$-separability of the complete preorder defined on the set of ghosts. Since we do not know how to describe all possible manners of inserting ghosts that preserve $d$-separability, we have lost control on the set of all possible strict (and nonstrict) unit representations in the uncountable case. Hence, the question of the uniqueness of a unit representation in the uncountable case remains open.

There is also a number of questions related to previous work by several authors that require further investigation.

Candeal et al. (2012, Th. 4.11) proved that, for a semiorder, s-separability is equivalent to any of a series of conditions guaranteeing separability for interval orders. These conditions were introduced in Oloriz et al. (1998) and further studied in Bosi et al. (2001) (see also the analysis of separability conditions for biorders in Doignon et al. (1984) and Nakamura (2002)). It would be useful to examine these conditions, equivalent to $s$-separablility for semiorders, and see whether some of them, by way of factorization, could lead to sharper characterization results.

A second issue of interest is related to the kind of real interval representations of biorders, interval orders and semiorders introduced and studied by Nakamura (2002). In these representations, each object is associated an interval, but some objects can be assigned an open one while others are assigned a closed one. The author characterizes the biorders, interval orders and semiorders that admit such "mixed" representations. For semiorders, the particular case of unit length mixed representations is not specifically investigated. Therefore, characterizing the semiorders that admit unit length mixed representations is still an open question. In his study of dense threshold structures, Narens (1994) has given a characterization of semiorders that are isomorphic to the classical semiorder on the reals or the rationals. More generally, one may want to characterize semiorders that are isomorphic to an interval (bounded or not) of the reals or the rationals, endowed with the restriction of the classical semiorder on the reals. The proof technique 
used in the present paper (and its companion Bouyssou and Pirlot, 2020a) can be adapted to establish characterizations for such semiorders. This will be the topic of a future paper.

We also expect that the same idea of proof could be beneficial for the characterization of the semiorders that admit a continuous unit representation (an issue that has received attention (Campíon et al., 2008, Gensemer, 1987a,b, 1988) but seems not to be completely solved).

\section{References}

F. Aleskerov, D. Bouyssou, and B. Monjardet. Utility maximization, choice and preference. Studies in Economic Theory, Volume 16. Springer-Verlag, Berlin, 2nd edition, 2007.

B. Balof, J.-P. Doignon, and S. Fiorini. The representation polyhedron of a semiorder. Order, 30(1):103-135, Mar 2013.

A. F. Beardon, J. C. Candeal, G. Herden, E. Induráin, and G. B. Mehta. The nonexistence of a utility function and the structure of non-representatble preference relations. Journal of Mathematical Economics, 37(1):17-38, 2002.

A. Beja and I. Gilboa. Numerical representation of imperfectly ordered preferences: a unified geometric exposition. Journal of Mathematical Psychology, 36:426-449, 1992.

B. Bosi, J. C. Candeal, E. Induráin, E. Oloriz, and M. Zudaire. Numerical representations of interval orders. Order, 18(2):171-190, 2001.

D. Bouyssou and M. Pirlot. Unit representation of semiorders. I: Countable sets. Working Paper, CNRS-LAMSADE, Université Paris-Dauphine, France, and University of Mons, Belgium, 2020a.

D. Bouyssou and M. Pirlot. A note on Candeal and Induráin's semiorder separability condition. In G. Bosi, M.J. Campión, J.C. Candeal, and E. Induráin, editors, Mathematical Topics on Representations of Ordered Structures and Utility Theory: Essays in Honor of Professor Ghanshyam B. Mehta, pages 129-141. Springer International Publishing, 2020b.

D. S. Bridges and G. B. Mehta. Representations of Preferences Orderings. Lecture Notes in Economics and Mathematical Systems 422. Springer-Verlag Berlin Heidelberg, 1st edition, 1995.

M. J. Campíon, J. C. Candeal, E. Induráin, and M. Zudaire. Continuous representability of semiorders. Journal of Mathematical Psychology, 52(1):48-54, 2008.

J. C. Candeal and E. Induráin. Semiorders and thresholds of utility discrimination: Solving the Scott-Suppes representability problem. Journal of Mathematical Psychology, 54(6):485-490, 2010.

J. C. Candeal, A. Estevan, J. Gutiérrez García, and E. Induráin. Semiorders with separability properties. Journal of Mathematical Psychology, 56:444-451, 2012. doi: 10.1016/j.jmp.2013.01.003. 
J.-P. Doignon. Sur les représentations minimales des semiordres et des ordres d'intervalles. Mathématiques et Sciences humaines, 101:49-59, 1988. URL http: //www. numdam. org/item/MSH_1988_-101_49_0.

J.-P. Doignon, A. Ducamp, and J.-C. Falmagne. On realizable biorders and the biorder dimension of a relation. Journal of Mathematical Psychology, 28(1):73-109, 1984.

S. H. Gensemer. On relationships between numerical representations of interval orders and semiorders. Journal of Economic Theory, 43:157-169, 1987a.

S. H. Gensemer. Continuous semiorder representations. Journal of Mathematical Economics, 16(3):275-289, 1987b.

S. H. Gensemer. On numerical representations of semiorders. Mathematical Social Sciences, 15(3):277-286, 1988.

Alfio Giarlotta and Stephen Watson. Universal semiorders. Journal of Mathematical Psychology, 73:80-93, 2016.

D.H. Krantz, R.D. Luce, P. Suppes, and A. Tversky. Foundations of Measurement, volume 1: Additive and Polynomial Representations. Academic Press, New York, 1971.

R. D. Luce. Semiorders and a theory of utility discrimination. Econometrica, 24(2): 178-191, 1956.

K. L. Manders. On JND representations of semiorders. Journal of Mathematical Psychology, 24(3):224-248, 1981.

Y. Nakamura. Real interval representations. Journal of Mathematical Psychology, 46 (2):140-177, 2002.

L. Narens. The measurement theory of dense threshold structures. Journal of Mathematical Psychology, 38(3):301-321, 1994.

E. Oloriz, J. C. Candeal, and E. Induráin. Representability of interval orders. Journal of Economic Theory, 78(1):219-227, 1998.

M. Pirlot. Minimal representation of a semiorder. Theory and Decision, 28:109-141, 1990.

M. Pirlot. Synthetic description of a semiorder. Discrete Applied Mathematics, 31: 299-308, 1991.

M. Pirlot and Ph. Vincke. Semiorders: properties, representations, applications. Kluwer Academic, 1997.

F. S. Roberts and Ch. H. Franke. On the theory of uniqueness in measurement. Journal of Mathematical Psychology, 14:211-218, 1976.

F.S. Roberts. Measurement theory, with applications to Decision Making, Utility and the Social Sciences. Addison-Wesley, Boston, 1979.

D. Scott and P. Suppes. Foundational aspects of theories of measurement. Journal of Symbolic Logic, 23(2):113-128, 061958. 